YITP-SB-12-25

\title{
Antiperiodic dynamical 6-vertex model I: Complete spectrum by SOV, matrix elements of the identity on separate states and connections to the periodic 8-vertex model
}

\author{
G. Niccoli1
}

\begin{abstract}
The spin-1/2 highest weight representations of the dynamical 6-vertex and the standard 8-vertex Yang-Baxter algebra on a finite chain are considered in this paper. In particular, the integrable quantum models associated to the corresponding transfer matrices under antiperiodic boundary conditions for the dynamical 6-vertex and periodic boundary conditions for the 8-vertex case are here analyzed.

For the antiperiodic dynamical 6-vertex transfer matrix defined on chains with an odd number of sites, we adapt the Sklyanin's quantum separation of variable (SOV) method and explicitly construct the SOV representations from the original space of the representations. In this way we provide the complete characterization of the eigenvalues and the eigenstates proving also the simplicity of its spectrum. Moreover, we characterize the matrix elements of the identity on separated states of this model by determinant formulae. The matrices entering in these determinants have elements given by sums over the SOV spectrum of the product of the coefficients of the separate states. This SOV analysis is done without any need to be reduced to the case of the so-called elliptic roots of unit and the results here derived define the required setup to extend to the dynamical 6-vertex model the approach recently developed in [1]-[5] to compute the form factors of the local operators in the SOV framework, these results will be presented in a future publication.

For the periodic 8 -vertex transfer matrix, we prove that its eigenvalues have to satisfy a fixed system of equations. In the case of a chain with an odd number of sites, this system of equations is the same entering in the SOV characterization of the antiperiodic dynamical 6-vertex transfer matrix spectrum. This implies that the set of the periodic 8-vertex eigenvalues is contained in the set of the antiperiodic dynamical 6-vertex eigenvalues. A criterion is introduced to find simultaneous eigenvalues of these two transfer matrix and associate to any of such eigenvalues one nonzero eigenstate of the periodic 8 -vertex transfer matrix by using the SOV results. Moreover, a preliminary discussion on the degeneracy occurring for odd chains in the periodic 8 -vertex transfer matrix spectrum is also presented.
\end{abstract}

\footnotetext{
${ }^{1}$ YITP, Stony Brook University, New York 11794-3840, USA, niccoli@max2.physics.sunysb.edu
} 


\section{Contents}

1 Introduction $\quad 3$

2 The dynamical 6-vertex models: spectrum and elementary matrix elements 5

2.1 The dynamical 6 -vertex models . . . . . . . . . . . . . . . . . . 5

2.1.1 Representation spaces of dynamical and spin operators . . . . . . . . . . . . . 5

2.1.2 Representations of the dynamical 6 -vertex models $\ldots \ldots \ldots \ldots$

2.1 .3 Quantum determinant ......................... 8

2.1.4 Antiperiodic dynamical 6 -vertex representations . . . . . . . . . . . . . . 10

2.1.5 Invariant subspace under antiperiodic 6 VD-generators $\ldots \ldots \ldots \ldots \ldots$

2.2 SOV-representations for $\overline{\mathcal{T}}^{(6 \mathrm{VD})}$-spectral problem $\ldots \ldots \ldots \ldots \ldots \ldots$

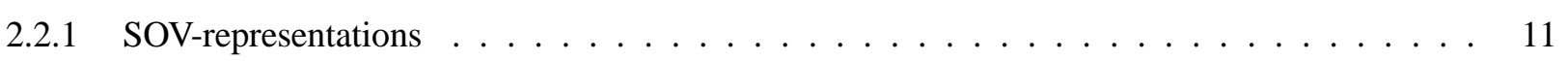

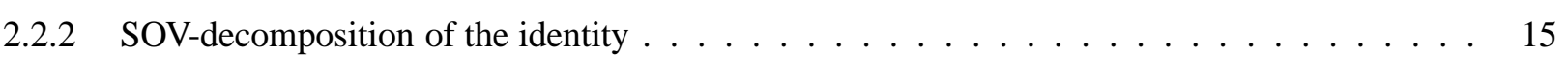

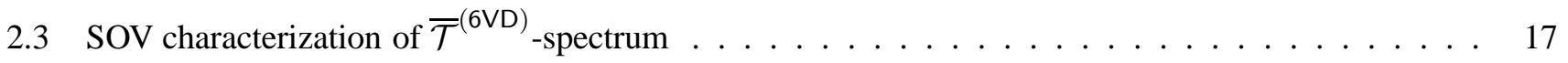

2.4 Action of left separate states on right separate states . . . . . . . . . . . . . . . . . 19

2.5 Decomposition of the identity in left and right separate basis . . . . . . . . . . . . . 20

3 On the periodic 8-vertex spectrum and connection with SOV

3.1 The 8 -vertex model . . . . . . . . . . . . . . . . . . . . . 21

3.2 Elementary properties of the periodic 8 -vertex transfer matrix $\ldots \ldots \ldots \ldots 21$

3.3 On the periodic 8 -vertex transfer matrix eigenvalues . . . . . . . . . . . . . . . 23

3.4 Gauge transformation from 8 -vertex to dynamical 6 -vertex models . . . . . . . . . . . . . 24

3.5 On the periodic 8 -vertex transfer matrix spectrum by SOV . . . . . . . . . . . . 26

3.5.1 Connections between periodic 8-vertex and antiperiodic dynamical 6-vertex spectrum . . 26

3.5.2 On the periodic 8 -vertex transfer matrix spectrum by SOV in odd chains . . . . . . . . . 2 27

4 Conclusion $\quad 28$

\begin{tabular}{ll} 
A Appendix & 29 \\
\hline
\end{tabular}

A.1 Spectrum of $\mathrm{T}^{(8 \mathrm{~V})}(\lambda)$ and $\overline{\mathcal{T}}^{(6 \mathrm{VD})}(\lambda \mid \tau)$ for $\mathrm{N}=1 \ldots \ldots \ldots \ldots \ldots$

A.2 Spectrum of $\mathrm{T}^{(8 \mathrm{~V})}(\lambda)$ and $\overline{\mathcal{T}}^{(6 \mathrm{VD})}(\lambda \mid \tau)$ for $\mathrm{N}=3 \ldots \ldots \ldots \ldots \ldots \ldots$

A.2.1 General statements on the spectrum for $\mathrm{N}=3 \ldots \ldots \ldots$

A.2.2 Some numerical data for $\mathrm{N}=3 \ldots \ldots \ldots \ldots \ldots \ldots$

References 


\section{Introduction}

In this paper we analyze two classes of lattice integrable quantum models characterized in the quantum inverse scattering method (QISM) [6]-[19] by monodromy matrices which are solutions of the dynamical Yang-Baxter equation w.r.t. the 6-vertex dynamical R-matrix and of the (standard) Yang-Baxter equation w.r.t. the 8-vertex Rmatrix, respectively. The representation theory of the dynamical 6-vertex Yang-Baxter algebra was introduced by Felder in [20] by the so-called theory of the elliptic quantum groups, see also [21]. There, it was recognized that the known Boltzmann weights defining the SOS (solid on solid) statistical models [22] when opportunely reorganized in a $4 \times 4$ matrix define the R-matrix solution of the dynamical 6-vertex Yang-Baxter algebra. The prototypical elements in these classes of integrable quantum models are constructed by defining representations of the corresponding monodromy matrices on chains of 2-dimensional representations (spin-1/2 quantum chains). Under homogeneous limits these representations define in the dynamical 6-vertex case the SOS model [22], [23]-[26] while in the 8vertex case the spin-1/2 XYZ quantum chain [27]-[35]. It is worth recalling that the monodromy matrices of these models are related by the Baxter's intertwining vectors and the spectral problems of the transfer matrices under periodic boundary conditions have been analyzed by Bethe ansatz and Q-operator techniques 2 in the case of chains with an even number of sites. In [23, 22, 24], R. Baxter has introduced the intertwining vectors, also called gauge transformation 3 , in order to be able to use Bethe ansatz techniques to analyzed the spectral problem (eigenvalues \& eigenstates) of the 8-vertex transfer matrix reducing it to one of 6-vertex type. The use of gauge transformations allows in particular to overcome the problem of the absence of reference states opening the possibility to analyze the 8-vertex spectral problem by using the algebraic Bethe ansatz (ABA) [6]-[7], as pioneered by Faddeev and Takhtajan in [8] while ABA analysis for the SOS model with periodic boundary conditions has been developed in [25]. However, it is worth remarking that, a part the general problem related to the proof of the completeness of the spectrum description 4 , the analysis by Bethe ansatz methods of the spectrum of these models leads to the introduction of two constrains. The first constrain is on the number of sites of the quantum chains which has to be even. This is required to obtain the commutativity of the dynamical 6-vertex transfer matrix which holds only for the reduction to the total spin zero-eigenspace under periodic boundary conditions. The second constrain is on the allowed values of the coupling constant $\eta$ of the 8-vertex transfer matrix which has to be restricted to the so-called cyclic values or elliptic roots of unit (i.e. when $\eta$ belongs to an integer square lattice with steps the periods of the theta functions). This is required in order to construct 8-vertex transfer matrix eigenstates by finite sums of the dynamical 6-vertex ones. In addition to the previously described constrains in the algebraic Bethe ansatz framework the lack of a scalar product analogue to the Slavnov's formula [44, 45, 46] is the first fundamental missing step toward the computation of matrix elements of local operators. It is then clear the need to overcome these problems in order to compute correlation functions.

In the present paper we implement a modified version of Sklyanin's quantum separation of variables (SOV) [47][49]. In particular, we derive the complete characterizations of the spectrum of the antiperiodid 5 dynamical 6-vertex transfer matrix defined on chains with an odd number of sites. Moreover, we compute the matrix elements of the identity for general separate states $\sqrt{6}$ which apply in particular for the eigenstates of the antiperiodic dynamical

\footnotetext{
${ }^{2}$ For the Q-operator construction see [22, 36] and also the series of papers [37]-[42].

${ }^{3}$ Let us comment that historically R. Baxter has used a vectorial representation for these transformations, e.g. see equation (3.3) of [22], which explains the original use of the terminology intertwining vectors. Here, we use the terminology gauge transformations, also used in [8], to refer to the matrix representation of the same transformations presented in 3.39].

${ }^{4}$ In fact, for the periodic 8-vertex transfer matrices the completeness of the spectrum description is verified only by some numerical analysis [43].

${ }^{5}$ This quantum integrable model has been introduced in [50].

${ }^{6}$ See Section 2.4 for the definition.
} 
6-vertex transfer matrix. Let us comment that the existing results [51, 52] for the antiperiodic dynamical 6-vertex model are mainly restricted to the construction of the functional separation of variables of Sklyanin. In this functional version an SOV representation of the dynamical 6-vertex Yang-Baxter algebra is defined on a space of symmetric functions leading only to the description of the wave functions of the transfer matrix eigenstates. In fact, the explicit construction of the SOV representation as well as of the transfer matrix eigenstates in the original representation space of the quantum chain were so far missing.

For the periodic 8-vertex transfer matrix, we prove that the set of all its eigenvalues is contained in the set of the solutions to an inhomogeneous system of $N$ quadratic equations in $N$ unknown, where $N$ is the number of sites of the chain. In the case $N$ odd this system coincides with the one entering in the SOV characterization of the antiperiodic dynamical 6-vertex transfer matrix spectrum and so the set of the periodic 8-vertex eigenvalues is proven to be contained in the antiperiodic dynamical 6-vertex one. Let us recall that the analysis of the odd $\mathrm{N}$ case is of particular interest for the periodic 8-vertex transfer matrix as in this case the Bethe ansatz analysis of [23, 22, 24, 8] does not apply. We use the Baxter's gauge transformations to further relate the periodic 8-vertex and the antiperiodic dynamical 6-vertex transfer matrices, allowing to analyze the 8-vertex spectral problem in terms

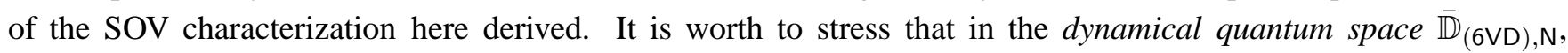
characterized by the condition that the antiperiodic dynamical 6-vertex transfer matrix is a one parameter family of commuting operators, these gauge transformations are not invertible operators. Nevertheless, we are able to use them to get a sufficient criterion which allows us to select simultaneous eigenvalues of the antiperiodic dynamical 6-vertex and the periodic 8-vertex transfer matrix and to associate to any one of these eigenvalues one corresponding nonzero 8 -vertex eigenstate. In the paper we will explain as the non invertible character of the gauge transformations on the dynamical quantum space $\overline{\mathbb{D}}_{(6 \mathrm{VD}), N}$ is a natural requirement as these transformations link transfer matrices with different degeneracy properties. Indeed, while the antiperiodic dynamical 6-vertex transfer matrix is proven here to have simple spectrum the periodic 8-vertex one has degeneracy even for completely general inhomogeneities. A preliminary analysis of this degeneracy issue is here presented by analyzing explicitly the periodic 8-vertex transfer matrix spectrum for chains with one and three sites.

The results derived in the present paper represent the first fundamental step in an approach to solve integrable quantum models which can be considered as the generalization to the SOV framework of the Lyon group method [46], [53]-[68]. The use of SOV is a strength point of our approach as it works for a large class of integrable quantum models, under simple conditions it leads to the complete construction of both the eigenvalues and the eigenstates of the transfer matrix and the simplicity of the spectrum can be easily shown in this framework. Moreover, the analysis developed in the present paper and that implemented previously in [1]-[5] suggest that this approach can lead to an universal representation of both the spectrum and the dynamics of a class of integrable quantum models which were not entirely solvable with other method 7 . Indeed, this is the case for all the key integrable quantum models analyzed so far in [1]-[5]. More in detail, in [3] and [4], the XXZ spin-1/2 quantum chain [27]-[35] and the higher spin-s XXX quantum chain?, both under antiperiodic boundary conditions, have been characterized and the form factors of the local spin operators have been represented in a determinant form. Similar results for the form factors have been obtained previously by this approach in [1, 2] for the lattice quantum sine-Gordon model [7, 19], the chiral Potts model [78]-[89] and the $\tau_{2}$-model [90]. These results are obtained by using as background the complete

\footnotetext{
${ }^{7}$ Like the algebraic Bethe ansatz, the coordinate Bethe ansatz [28], [69] and [70], the Baxter Q-operator method [69] and the analytic Bethe ansatz [71]-[72].

${ }^{8}$ Let us comment that previous results on this model with antiperiodic boundary conditions were mainly given by the Q-operator construction [73] and the functional separation of variables [48, 74]. Moreover see [75] for the eigenvalue analysis of the XXZ spin-1/2 chain with general antiperiodic boundary conditions by a functional method based on the Yang-Baxter algebra; method also applies to open chain for general integrable boundary conditions.

${ }^{9}$ Instead in the periodic chain matrix elements of local operators were compute in the ABA framework in [76 77].
} 
SOV spectrum characterization constructed in [91]-[93] for the lattice quantum sine-Gordon model and in 10 [98] for the general cyclic representations of the 6-vertex Yang-Baxter algebra corresponding to the $\tau_{2}$-model and the chiral Potts model. Moreover, in [5] the SOV setup has been implemented and the matrix elements of some interesting quasi-local string of local operators have been computed for the integrable quantum model associated to the spin-1/2 representations of the reflection algebra [99]-[106], under quite general non-diagonal boundary conditions. In all these models the matrix elements of local operators on separate states are characterized by determinant formulae written as simple modifications of those of the identity. The main differences in all these formulae are only due to model dependent features, like the nature of the spectrum of the quantum separate variables and the form of the SOV reconstruction of local operators. Let us comment that in the literature there exist previous results on matrix elements of local operators which even if developed by different approaches made use of quantum separation of variables. The results in Smirnov's paper [107] are of special interest; there, for the quantum integrable Toda chain [47], the form factors of a conjectured 11 basis of local operators are derived in Sklyanin's SOV framework by determinant formulae which confirm the universal picture outlined. It is also worth pointing out that the form factor 12 of the restricted sine-Gordon model at the reflectionless points in the S-matrix formulation 13 [119, 120] admit once again determinant representations and the connection with SOV is established on the basis of the semi-classical analysis of [119], used there also as a tool to overcome the problem 14 of the local field identification.

\section{The dynamical 6-vertex models: spectrum and elementary matrix elements}

\subsection{The dynamical 6-vertex models}

In the following, we introduce an operator $\tau$ whose eigenvalues on the space of the representation coincide with the dynamical parameter $t$. The aim is to recover a separate description for the dynamical parameter which will be particularly useful in the SOV description of the antiperiodic dynamical 6-vertex spectral problem.

\subsubsection{Representation spaces of dynamical and spin operators}

Let us introduce a couple of dynamical operators $\tau$ and $\mathrm{T}_{\tau}^{ \pm}$which satisfy the following commutation relations:

$$
\mathbf{T}_{\tau}^{ \pm} \tau=(\tau \pm \eta) \mathbf{T}_{\tau}^{ \pm}
$$

and N copies of (local spin) $s l(2)$ generators $S_{n}^{a}$ and let us impose the following commutation relations:

$$
\left[S_{n}^{a}, S_{m \neq n}^{b}\right]=\left[S_{n}^{a}, \tau\right]=\left[S_{n}^{a}, \mathrm{~T}_{\tau}^{ \pm}\right]=0 \quad \forall n, m \in\{1, \ldots, \mathrm{N}\} \text { and } a, b=x, y, z
$$

\footnotetext{
${ }^{10}$ See [94]-[97] for a first analysis by SOV method of the $\tau_{2}$-model and some results on form factors in the restricted case of the generalized Ising model.

${ }^{11}$ There this conjecture is required by the absence of a direct SOV reconstruction of local operators. Later a reconstruction has been given in [108] w.r.t. a new set of quantum separate variables defined by a change of variables on the original Sklyanin's ones.

${ }^{12}$ Form factors which have been rederived in [109] also by exploiting previous results established in the series of papers [110]-[113] for the infinite volume limit of the XXZ spin-1/2 chain.

${ }^{13}$ See [114]-[118] and references therein.

${ }^{14}$ This is a longstanding problem in the S-matrix formulation which has been so far addressed by exploiting the description [121]-[124] of massive IQFTs as (superrenormalizable) perturbations of conformal field theories [125]-[129] by relevant local fields. Several results are known which allows to classify the local fields of massive theories (i.e. the solutions to the form factor equations [130]-[145]) in terms of those of the ultraviolet conformal field theories, see for example [146]-[149] and the series of works [150]-[155].
} 
Then the space of the representation of these dynamical and spin operators can be chosen as it follows:

$$
\mathbb{D}_{(6 \mathrm{VD}), \mathrm{N}} \equiv \mathbb{D}_{\mathrm{N}} \otimes \underbrace{\mathbb{C}^{2} \otimes \otimes \otimes \mathbb{C}^{2}}_{\mathrm{N}}
$$

where $\mathbb{D}_{\mathrm{N}}$ is the space of the representation of the dynamical operators which is infinite dimensional in our definition 15 . Here, we have chosen for all the $s l(2)$ generators the spin-1/2 representation; i.e. a 2-dimensional local quantum space $\mathbb{C}^{2}$ is associated to any site of the chain and the local spin generators are represented by the $2 \times 2$ Pauli matrices $\sigma_{n}^{a}$.

Moreover, we introduce the following definition of left (covectors) and right (vectors) $\tau$-eigenbasis of $\mathbb{D}_{\mathrm{N}}^{\mathcal{L}}$ and $\mathbb{D}_{\mathrm{N}}^{\mathcal{R}}$, respectively:

$$
\left\langle t(a)\left|\equiv\left\langle t(0)\left|\mathbf{T}_{\tau}^{a}, \quad\right| t(a)\right\rangle \equiv \mathbf{T}_{\tau}^{-a}\right| t(0)\right\rangle, \quad \forall a \in \mathbb{Z},
$$

with:

$$
\langle t(a)|\tau=t(a)\langle t(a)|, \quad \tau| t(a)\rangle=t(a)| t(a)\rangle, \quad t(a) \equiv-\frac{\eta}{2} a \quad \forall a \in \mathbb{Z},
$$

where we fix $\langle t(a) \mid t(b)\rangle=\delta_{a, b}, \forall a, b \in \mathbb{Z}$. Moreover, defined the following left and right spin basis:

$$
\left\langle n, h_{n}\left|\sigma_{n}^{z}=\left(1-2 h_{n}\right)\left\langle n, h_{n}\left|, \quad \sigma_{n}^{z}\right| n, h_{n}\right\rangle=\left(1-2 h_{n}\right)\right| n, h_{n}\right\rangle, \quad h_{n} \in\{0,1\},
$$

with $\left\langle n, h_{n} \mid n, h_{n}^{\prime}\right\rangle=\delta_{h_{n}, h_{n}^{\prime}}$, in each local quantum spin chain of the representation, we can introduce the left and right dynamical-spin basis in $\mathbb{D}_{(6 \mathrm{VD}), \mathrm{N}}^{\mathcal{L}}$ and $\mathbb{D}_{(6 \mathrm{VD}), \mathrm{N}}^{\mathcal{R}}$, respectively:

$$
\otimes_{n=1}^{\mathrm{N}}\left\langle n, h_{n}\left|\otimes\left\langle t(a)\left|, \quad \otimes_{n=1}^{\mathrm{N}}\right| n, h_{n}\right\rangle \otimes\right| t(a)\right\rangle,
$$

composed of common eigenstates of the commuting operators $\tau$ and $\sigma_{n}^{z}$. A scalar product is introduced in the space $\mathbb{D}_{(6 \mathrm{VD}), \mathrm{N}}^{\mathcal{R}}$ by defining its action on the elements of the dynamical-spin basis:

$$
\left(\otimes_{n=1}^{\mathrm{N}}\left|n, h_{n}\right\rangle \otimes|t(a)\rangle, \otimes_{n=1}^{\mathrm{N}}\left|n, h_{n}^{\prime}\right\rangle \otimes\left|t\left(a^{\prime}\right)\right\rangle\right)=\delta_{a, a^{\prime}} \prod_{n=1}^{\mathrm{N}} \delta_{h_{n}, h_{n}^{\prime}} .
$$

Note that we have defined the representation in a way that the spectrum (eigenvalues) of the operator $\tau$ contains that of $-\eta S / 2$ where:

$$
\mathrm{S}=\sum_{n=1}^{\mathrm{N}} \sigma_{n}^{z}
$$

is the total $z$-component of the spin; the reason for that will be clear in the following.

\footnotetext{
${ }^{15}$ Note that in the root of unit case, we can define also a cyclic representation for the operator $\tau$. This point in the present formalism will be described elsewhere anyhow for the antiperiodic chain this cyclicity condition does not play a fundamental role as it was in the periodic case for the application of algebraic Bethe ansatz to the 8-vertex model.
} 


\subsubsection{Representations of the dynamical 6-vertex models}

Let us define the elliptic dynamical 6-vertex R-matrix 16 :

$$
R_{0 a}^{(6 \mathrm{VD})}(\lambda \mid \tau)=\left(\begin{array}{cccc}
a(\lambda) & 0 & 0 & 0 \\
0 & b(\lambda \mid \tau) & c(\lambda \mid \tau) & 0 \\
0 & c(\lambda \mid-\tau) & b(\lambda \mid-\tau) & 0 \\
0 & 0 & 0 & a(\lambda)
\end{array}\right)
$$

where $a(\lambda), b(\lambda \mid \tau)$ and $c(\lambda \mid \tau)$ are defined by:

$$
a(\lambda)=\theta(\lambda+\eta), \quad b(\lambda \mid \tau)=\frac{\theta(\lambda) \theta(\tau+\eta)}{\theta(\tau)}, \quad c(\lambda \mid \tau)=\frac{\theta(\eta) \theta(\tau+\lambda)}{\theta(\tau)},
$$

here and in the following we use the notation:

$$
\theta(\lambda)=\theta_{1}(\lambda \mid \omega)
$$

where 17 $\theta_{1}(\lambda \mid \omega)$ is the standard theta-1 elliptic function of modular parameter $\omega$. Then, the R-matrix $R_{1,2}^{(6 \mathrm{VD})}(\lambda \mid \tau)$ is solution of the following dynamical Yang-Baxter equation:

$$
R_{1,2}^{(6 \mathrm{VD})}\left(\lambda_{12} \mid \tau+\eta \sigma_{a}^{z}\right) R_{1, a}^{(6 \mathrm{VD})}\left(\lambda_{1} \mid \tau\right) R_{2, a}^{(6 \mathrm{VD})}\left(\lambda_{2} \mid \tau+\eta \sigma_{1}^{z}\right)=R_{2, a}^{(6 \mathrm{VD})}\left(\lambda_{2} \mid \tau\right) R_{1, a}^{(6 \mathrm{VD})}\left(\lambda_{1} \mid \tau+\eta \sigma_{2}^{z}\right) R_{1,2}^{(6 \mathrm{VD})}\left(\lambda_{12} \mid \tau\right),
$$

where $\lambda_{12} \equiv \lambda_{1}-\lambda_{2}$. It is possible to introduce the following dynamical 6-vertex monodromy matrix:

$$
\mathrm{M}_{0}^{(6 \mathrm{VD})}(\lambda \mid \tau) \equiv R_{0, \mathrm{~N}}^{(6 \mathrm{VD})}\left(\lambda-\xi_{\mathrm{N}} \mid \tau+\eta \sum_{a=1}^{\mathrm{N}-1} \sigma_{a}^{z}\right) \cdots R_{0,1}^{(6 \mathrm{VD})}\left(\lambda-\xi_{1} \mid \tau\right) \equiv\left(\begin{array}{cc}
\mathrm{A}(\lambda \mid \tau) & \mathrm{B}(\lambda \mid \tau) \\
\mathrm{C}(\lambda \mid \tau) & \mathrm{D}(\lambda \mid \tau)
\end{array}\right)_{0},
$$

where the $\xi_{n}$ for $n \in\{1, \ldots,-\mathrm{N}\}$ are parameters of the model called inhomogeneities. Then this monodromy matrix is a solution of the same type of dynamical Yang-Baxter equation:

$$
R_{1,2}^{(6 \mathrm{VD})}\left(\lambda_{12} \mid \tau+\eta \mathrm{S}\right) \mathrm{M}_{1}^{(6 \mathrm{VD})}\left(\lambda_{1} \mid \tau\right) \mathrm{M}_{2}^{(6 \mathrm{VD})}\left(\lambda_{2} \mid \tau+\eta \sigma_{1}^{z}\right)=\mathrm{M}_{2}^{(6 \mathrm{VD})}\left(\lambda_{2} \mid \tau\right) \mathrm{M}_{1}^{(6 \mathrm{VD})}\left(\lambda_{1} \mid \tau+\eta \sigma_{2}^{z}\right) R_{1,2}^{(6 \mathrm{VD})}\left(\lambda_{12} \mid \tau\right)
$$

where $S$ is the total $z$-component of the spin defined in (2.9). Moreover, it is worth remarking that the following commutation relations hold:

$$
[\mathrm{A}(\lambda \mid \tau), \tau]=[\mathrm{B}(\lambda \mid \tau), \tau]=[\mathrm{C}(\lambda \mid \tau), \tau]=[\mathrm{D}(\lambda \mid \tau), \tau]=0
$$

and

$$
[\mathrm{A}(\lambda \mid \tau), \mathrm{S}]=[\mathrm{D}(\lambda \mid \tau), \mathrm{S}]=0, \quad[\mathrm{C}(\lambda \mid \tau), \mathrm{S}]=-2 \mathrm{C}(\lambda \mid \tau), \quad[\mathrm{B}(\lambda \mid \tau), \mathrm{S}]=2 \mathrm{~B}(\lambda \mid \tau) .
$$

Note that defined:

$$
\mathrm{T}_{\tau}^{ \pm \sigma_{a}^{z}} \equiv\left(\begin{array}{cc}
\mathrm{T}_{\tau}^{ \pm} & 0 \\
0 & \mathrm{~T}_{\tau}^{\mp}
\end{array}\right)_{a}
$$

\footnotetext{
${ }^{16}$ The presentation of our results will be done directly in this elliptic case, however, it is interesting to remark that also the trigonometric case corresponding to the following choice of dynamical R-matrix:

$$
a(\lambda)=\sinh (\lambda+\eta), \quad b(\lambda \mid \tau)=\frac{\sinh \lambda \sinh (\tau+\eta)}{\sinh \tau}, \quad c(\lambda \mid \tau)=\frac{\sinh \eta \sinh (\tau+\lambda)}{\sinh \tau}
$$

can be similarly described in our approach.

${ }^{17}$ In this paper the $\theta_{i}(\lambda \mid \omega)$ for $i \in\{1, . .4\}$ are the standard theta functions as defined for example at page 877 of [158], where we are using for the argument of these functions $(\lambda \mid \omega)$ instead of $(u \mid \tau)$.
} 
we can rewrite the dynamical Yang-Baxter equations in the following form:

$$
R_{1,2}^{(6 \mathrm{VD})}\left(\lambda_{12} \mid \tau+\eta \mathrm{S}\right) \mathrm{M}_{1}^{(6 \mathrm{VD})}\left(\lambda_{1} \mid \tau\right) \mathrm{T}_{\tau}^{\sigma_{1}^{z}} \mathrm{M}_{2}^{(6 \mathrm{VD})}\left(\lambda_{2} \mid \tau\right) \mathrm{T}_{\tau}^{-\sigma_{1}^{z}}=\mathrm{M}_{2}^{(6 \mathrm{VD})}\left(\lambda_{2} \mid \tau\right) \mathrm{T}_{\tau}^{\sigma_{2}^{z}} \mathrm{M}_{1}^{(6 \mathrm{VD})}\left(\lambda_{1} \mid \tau\right) \mathrm{T}_{\tau}^{-\sigma_{2}^{z}} R_{1,2}^{(6 \mathrm{VD})}\left(\lambda_{12} \mid \tau\right) .
$$

Let us remark that while the dynamical 6-vertex generators $\mathrm{B}(\lambda \mid \tau)$ and $\mathrm{C}(\lambda \mid \tau)$ are nilpotent operators of order $\mathrm{N}+1$, for a chain of size $\mathrm{N}$, the generators $\mathrm{A}(\lambda \mid \tau)$ and $\mathrm{D}(\lambda \mid \tau)$ are not nilpotent operators. So, from the form of the dynamical 6-vertex Yang-Baxter commutation relations, it is clear that the set spanned by the dynamical parameter $t$ (the eigenvalues of $\tau$ ) is always an infinite lattice of step $\eta$. We can also define the following monodromy matrix:

$$
\left(\begin{array}{ll}
\mathcal{A}(\lambda \mid \tau) & \mathcal{B}(\lambda \mid \tau) \\
\mathcal{C}(\lambda \mid \tau) & \mathcal{D}(\lambda \mid \tau)
\end{array}\right)_{0}=\mathcal{M}_{0}^{(6 \mathrm{VD})}(\lambda \mid \tau) \equiv \mathrm{M}_{0}^{(6 \mathrm{VD})}(\lambda \mid \tau) \mathrm{T}_{\tau}^{\sigma_{0}^{z}}
$$

note that the $\mathcal{X}(\lambda \mid \tau)(\mathcal{X}=\mathcal{A}, \mathcal{B}, \mathcal{C}$ and $\mathcal{D})$ are operator functions of $\tau$ and $T_{\tau}^{ \pm}$but for simplicity we omit the explicit dependence from $T_{\tau}^{ \pm}$in their arguments. For this monodromy matrix the dynamical Yang-Baxter equation reads:

$$
R_{1,2}^{(6 \mathrm{VD})}\left(\lambda_{12} \mid \tau+\eta \mathrm{S}\right) \mathcal{M}_{1}^{(6 \mathrm{VD})}\left(\lambda_{1} \mid \tau\right) \mathcal{M}_{2}^{(6 \mathrm{VD})}\left(\lambda_{2} \mid \tau\right)=\mathcal{M}_{2}^{(6 \mathrm{VD})}\left(\lambda_{2} \mid \tau\right) \mathcal{M}_{1}^{(6 \mathrm{VD})}\left(\lambda_{1} \mid \tau\right) R_{1,2}^{(6 \mathrm{VD})}\left(\lambda_{12} \mid \tau\right)
$$

where we have used that:

$$
\mathrm{T}_{\tau}^{-\sigma_{1}^{z}} \mathrm{~T}_{\tau}^{-\sigma_{2}^{z}} R_{1,2}^{(6 \mathrm{VD})}\left(\lambda_{12} \mid \tau\right) \mathrm{T}_{\tau}^{\sigma_{1}^{z}} \mathrm{~T}_{\tau}^{\sigma_{2}^{z}}=R_{1,2}^{(6 \mathrm{VD})}\left(\lambda_{12} \mid \tau\right)
$$

Finally, let us comment that in [21] it was shown that the dynamical 6-vertex Yang-Baxter equations (2.13) are just the rewriting of the Baxter's star-triangle equations for the Boltzmann weights:

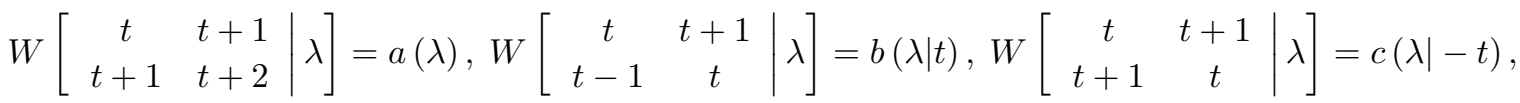

$$
\begin{aligned}
& W\left[\begin{array}{cc|}
t & t-1 \\
t-1 & t .2
\end{array} \mid \lambda\right]=a(\lambda), W\left[\begin{array}{cc}
t & t-1 \\
t+1 & t
\end{array} \mid \lambda\right]=b(\lambda \mid-t), W\left[\begin{array}{cc}
t & t-1 \\
t-1 & t
\end{array} \mid \lambda\right]=c(\lambda \mid t),
\end{aligned}
$$

of the solid-on-solid (SOS) mode 18 , where on the r.h.s. of the above equations there are the entries of the dynamical 6-vertex R-matrix.

\subsubsection{Quantum determinant}

A fundamental object to define in the dynamical 6-vertex Yang-Baxter algebra is the so-called quantum determinan 19 . In particular, it plays a fundamental role in the construction of the quantum separation of variables for these algebra as we will explain in the following.

Proposition 2.1. In the dynamical 6-vertex Yang-Baxter algebra, we can introduce the following central quantum determinant:

$$
\begin{aligned}
\operatorname{det}_{q} \mathrm{M}(\lambda) & \equiv \frac{\theta(\tau+\eta \mathrm{S})}{\theta(\tau)}(\mathrm{A}(\lambda \mid \tau) \mathrm{D}(\lambda-\eta \mid \tau+\eta)-\mathrm{B}(\lambda \mid \tau) \mathrm{C}(\lambda-\eta \mid \tau-\eta)) \\
& =\frac{\theta(\tau+\eta \mathrm{S})}{\theta(\tau)}(\mathrm{D}(\lambda \mid \tau) \mathrm{A}(\lambda-\eta \mid \tau-\eta)-\mathrm{C}(\lambda \mid \tau) \mathrm{B}(\lambda-\eta \mid \tau+\eta)) \\
& =\mathrm{A}(\lambda) \mathrm{D}(\lambda-\eta),
\end{aligned}
$$

\footnotetext{
${ }^{18}$ This model of statistical mechanics is defined on a square lattice and to each site $n$ a "height" $l_{n}$ is associated and the interactions are defined round each face (composed by 4 adjacent sites) of the lattice. These interactions are nonzero only for adjacent heights which differ by 1 and are described by the Boltzmann weights $W[\mid \lambda]$ of equations $(2.23)$ and (2.24).

${ }^{19}$ See [156] and the historical note [157] for a first proof of the centrality of the quantum determinant in the Yang-Baxter algebra.
} 
where:

$$
\mathrm{A}(\lambda) \equiv \prod_{n=1}^{\mathrm{N}} a\left(\lambda-\xi_{n}\right), \quad \mathrm{D}(\lambda) \equiv \mathrm{A}(\lambda-\eta)
$$

Moreover, the following inversion formula holds:

$$
\mathrm{M}_{0}^{(6 \mathrm{VD})}(\lambda \mid \tau)\left(\begin{array}{cc}
\mathrm{D}(\lambda-\eta \mid \tau+\eta) & -\mathrm{B}(\lambda-\eta \mid \tau+\eta) \\
-\mathrm{C}(\lambda-\eta \mid \tau-\eta) & \mathrm{A}(\lambda-\eta \mid \tau-\eta)
\end{array}\right)_{0} \frac{\theta(\tau+\eta \mathrm{S}) / \theta(\tau)}{\operatorname{det}_{q} \mathrm{M}(\lambda)}=\left(\begin{array}{ll}
1 & 0 \\
0 & 1
\end{array}\right)_{0}
$$

Proof. The proof follows by proving the statement for the generic quantum site $n$ and then showing that the product of the local quantum determinants reproduce the complete one. Let us introduce the notation:

$$
\begin{aligned}
\operatorname{det}_{q} R_{0 n}^{(6 \mathrm{VD})}(\lambda \mid \tau) & =\left(R_{0 n}^{(6 \mathrm{VD})}\right)_{11}(\lambda \mid \tau)\left(R_{0 n}^{(6 \mathrm{VD})}\right)_{22}(\lambda-\eta \mid \tau+\eta) \\
& -\left(R_{0 n}^{(6 \mathrm{VD})}\right)_{12}(\lambda \mid \tau)\left(R_{0 n}^{(6 \mathrm{VD})}\right)_{21}(\lambda-\eta \mid \tau-\eta),
\end{aligned}
$$

and

$$
\mathrm{S}_{n} \equiv \sum_{a=1}^{n} \sigma_{a}^{z}
$$

Then it is a simple exercise to verify the identity:

$$
\operatorname{det}_{q} R_{0, n}^{(6 \mathrm{VD})}\left(\lambda-\xi_{n} \mid \tau+\eta \mathrm{S}_{n-1}\right)=a\left(\lambda-\xi_{n}\right) a\left(\lambda-\xi_{n}-2 \eta\right) \frac{\theta\left(\tau+\eta \mathrm{S}_{n-1}\right)}{\theta\left(\tau+\eta \mathrm{S}_{n}\right)},
$$

once we use the formula20.

$$
\theta_{1}(x+y) \theta_{1}(x-y) \theta_{4}^{2}(0)=\theta_{3}^{2}(x) \theta_{2}^{2}(y)-\theta_{2}^{2}(x) \theta_{3}^{2}(y)=\theta_{1}^{2}(x) \theta_{4}^{2}(y)-\theta_{4}^{2}(x) \theta_{1}^{2}(y) .
$$

Now, by taking the product:

$$
\begin{aligned}
\mathrm{A}(\lambda) \mathrm{D}(\lambda-\eta) & =\prod_{n=1}^{\mathrm{N}} a\left(\lambda-\xi_{n}\right) a\left(\lambda-\xi_{n}-2 \eta\right) \\
& =\prod_{n=1}^{\mathrm{N}}\left[\frac{\theta\left(\tau+\eta \mathrm{S}_{n}\right)}{\theta\left(\tau+\eta \mathrm{S}_{n-1}\right)} \operatorname{det}_{q} R_{0, n}^{(6 \mathrm{VD})}\left(\lambda \mid \tau+\eta \mathrm{S}_{n-1}\right)\right] \\
& =\frac{\theta(\tau+\eta \mathrm{S})}{\theta(\tau)} \operatorname{det}_{q} R_{0, \mathrm{~N}}^{(6 \mathrm{VD})}\left(\lambda \mid \tau+\eta \mathrm{S}_{\mathrm{N}-1}\right) \cdots \operatorname{det}_{q} R_{0,2}^{(6 \mathrm{VD})}\left(\lambda \mid \tau+\eta \mathrm{S}_{1}\right) \operatorname{det}_{q} R_{0,1}^{(6 \mathrm{VD})}(\lambda \mid \tau) \\
& =\operatorname{det}_{q} \mathrm{M}(\lambda) .
\end{aligned}
$$

Finally, the inversion formula (2.29) follows from the quantum determinant formulae and from the identities:

$$
\begin{aligned}
& \mathrm{A}(\lambda \mid \tau) \mathrm{B}(\lambda-\eta \mid \tau+\eta)-\mathrm{B}(\lambda \mid \tau) \mathrm{A}(\lambda-\eta \mid \tau-\eta)=0 \\
& \mathrm{D}(\lambda \mid \tau) \mathrm{C}(\lambda-\eta \mid \tau-\eta)-\mathrm{C}(\lambda \mid \tau) \mathrm{D}(\lambda-\eta \mid \tau+\eta)=0
\end{aligned}
$$

which directly follows from the dynamical Yang-Baxter equations (2.15).

\footnotetext{
${ }^{20}$ See for example equation 7 at page 881 of [158].
} 


\subsubsection{Antiperiodic dynamical 6-vertex representations}

As we will show in the paper it is of particular interest to introduce an antiperiodic version of the dynamical 6-vertex model by introducing the following monodromy matrix:

$$
\overline{\mathrm{M}}_{0}^{(6 \mathrm{VD})}\left(\lambda_{1} \mid \tau\right) \equiv \sigma_{0}^{x} \mathrm{M}_{0}^{(6 \mathrm{VD})}\left(\lambda_{1} \mid \tau\right)
$$

then the dynamical Yang-Baxter equation reads:

$R_{1,2}^{(6 \mathrm{VD})}\left(\lambda_{12} \mid-\tau-\eta \mathrm{S}\right) \overline{\mathrm{M}}_{1}^{(6 \mathrm{VD})}\left(\lambda_{1} \mid \tau\right) \mathrm{T}_{\tau}^{\sigma_{1}^{z}} \overline{\mathrm{M}}_{2}^{(6 \mathrm{VD})}\left(\lambda_{2} \mid \tau\right) \mathrm{T}_{\tau}^{-\sigma_{1}^{z}}=\overline{\mathrm{M}}_{2}^{(6 \mathrm{VD})}\left(\lambda_{2} \mid \tau\right) \mathrm{T}_{\tau}^{\sigma_{2}^{z}} \overline{\mathrm{M}}_{1}^{(6 \mathrm{VD})}\left(\lambda_{1} \mid \tau\right) \mathrm{T}_{\tau}^{-\sigma_{2}^{z}} R_{1,2}^{(6 \mathrm{VD})}\left(\lambda_{12} \mid \tau\right)$,

being:

$$
\sigma_{1}^{x} \otimes \sigma_{2}^{x} R_{1,2}^{(6 \mathrm{VD})}(\lambda \mid y)=R_{1,2}^{(6 \mathrm{VD})}(\lambda \mid-y) \sigma_{1}^{x} \otimes \sigma_{2}^{x} .
$$

It is worth also to define the following monodromy matrix:

$$
\overline{\mathcal{M}}_{0}^{(6 \mathrm{VD})}(\lambda \mid \tau) \equiv \overline{\mathrm{M}}_{0}^{(6 \mathrm{VD})}(\lambda \mid \tau) \mathrm{T}_{\tau}^{\sigma_{0}^{z}}
$$

for it the dynamical Yang-Baxter equation reads:

$$
R_{1,2}^{(6 \mathrm{VD})}\left(\lambda_{12} \mid-\tau-\eta \mathrm{S}\right) \overline{\mathcal{M}}_{1}^{(6 \mathrm{VD})}\left(\lambda_{1} \mid \tau\right) \overline{\mathcal{M}}_{1}^{(6 \mathrm{VD})}\left(\lambda_{2} \mid \tau\right)=\overline{\mathcal{M}}_{1}^{(6 \mathrm{VD})}\left(\lambda_{2} \mid \tau\right) \overline{\mathcal{M}}_{1}^{(6 \mathrm{VD})}\left(\lambda_{1} \mid \tau\right) R_{1,2}^{(6 \mathrm{VD})}\left(\lambda_{12} \mid \tau\right)
$$

\subsubsection{Invariant subspace under antiperiodic 6VD-generators}

Let us define the operator:

$$
\mathrm{S}_{\tau} \equiv \eta \mathrm{S}+2 \tau
$$

in $\mathbb{D}_{(6 \mathrm{VD}), \mathrm{N}}$ then we denote with $\overline{\mathbb{D}}_{(6 \mathrm{VD}), \mathrm{N}}$ the $2^{\mathrm{N}}$-dimensional linear eigenspace corresponding to the eigenvalue zero $\mathrm{S}_{\tau}$; i.e. $\overline{\mathbb{D}}_{(6 \mathrm{VD}), \mathrm{N}}$ is the linear space defined by the condition that the eigenvalues of the commuting operators $-2 \tau$ and $\eta \mathrm{S}$ are coinciding. In terms of the dynamical-spin basis the linear (covector) space $\overline{\mathbb{D}}_{(6 \mathrm{VD}), \mathrm{N}}^{\mathcal{L}}$ is generated by the elements 21 :

$$
\otimes_{n=1}^{\mathrm{N}}\left\langle n, h_{n}\right| \otimes\left\langle t_{\mathbf{h}}\right|, \quad \text { where } t_{\mathbf{h}} \equiv-\frac{\eta}{2} \boldsymbol{s}_{\mathbf{h}}, \quad \mathbf{s}_{\mathbf{h}} \equiv \sum_{k=1}^{\mathrm{N}}\left(1-2 h_{k}\right) \text { and } \mathbf{h} \equiv\left(h_{1}, \ldots, h_{\mathrm{N}}\right),
$$

and the linear (vector) space $\overline{\mathbb{D}}_{(6 \mathrm{VD}), \mathrm{N}}^{\mathcal{R}}$ is generated by the elements:

$$
\otimes_{n=1}^{\mathrm{N}}\left|n, h_{n}\right\rangle \otimes\left|t_{\mathbf{h}}\right\rangle .
$$

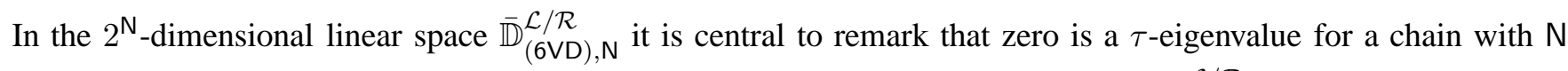
even while it is not for a chain with $N$ odd. This simple observation implies that $\overline{\mathbb{D}}_{(6 \mathrm{VD}), N}^{\mathcal{L} / \mathcal{R}}$ is not a well define representation space of the dynamical Yang-Baxter algebra for the presence of divergencies in (2.10) for the zero $\tau$-eigenvalue. On the contrary, in the case of an odd chain it holds:

Theorem 2.1. On the linear spaces $\overline{\mathbb{D}}_{(6 \mathrm{VD}), \mathrm{N}}^{\mathcal{L} / \mathcal{R}}$ are well defined left/right finite dimensional representations of the operators:

$$
\mathrm{A}(\lambda \mid \tau), \quad \mathrm{D}(\lambda \mid \tau), \quad \mathcal{B}(\lambda \mid \tau), \quad \mathcal{C}(\lambda \mid \tau)
$$

Moreover, the antiperiodic dynamical 6-vertex transfer matrix:

$$
\overline{\mathcal{T}}^{(6 \mathrm{VD})}(\lambda \mid \tau) \equiv \operatorname{tr}_{0} \overline{\mathcal{M}}_{0}^{(6 \mathrm{VD})}(\lambda \mid t)=\mathcal{B}(\lambda \mid \tau)+\mathcal{C}(\lambda \mid \tau)
$$

defines a one parameter family of commuting operators on $\overline{\mathbb{D}}_{(6 \mathrm{VD}), \mathrm{N}}^{\mathcal{L} / \mathcal{R}}$

\footnotetext{
${ }^{21}$ Note that we are using the simplified notation $t_{\mathbf{h}}$ instead of $t\left(\mathbf{s}_{\mathbf{h}}\right)$.
} 
Proof. To prove the first statement in the theorem we have to prove that the linear spaces $\overline{\mathbb{D}}_{(6 \mathrm{VD}), \mathrm{N}}^{\mathcal{L} / \mathcal{R}}$ are invariant under the action of the operators:

$$
\mathrm{A}(\lambda \mid \tau), \quad \mathrm{D}(\lambda \mid \tau), \quad \mathcal{B}(\lambda \mid \tau), \quad \mathcal{C}(\lambda \mid \tau)
$$

All what we need are the following commutation relations:

$$
\begin{array}{lll}
{[\mathrm{A}(\lambda \mid \tau), \mathrm{S}]} & =0, & {[\mathrm{~A}(\lambda \mid \tau), \tau]=0,} \\
{[\mathrm{~A}(\lambda \mid \tau), \mathrm{S}]} & =0, & {[\mathrm{~A}(\lambda \mid \tau), \tau]=0,} \\
{[\mathcal{B}(\lambda \mid \tau), \mathrm{S}]} & =2 \mathcal{B}(\lambda \mid \tau) & {[\mathcal{B}(\lambda \mid \tau), \tau]=-\eta \mathcal{B}(\lambda \mid \tau),} \\
{[\mathcal{C}(\lambda \mid \tau), \mathrm{S}]} & =-2 \mathcal{C}(\lambda \mid \tau),[\mathcal{C}(\lambda \mid \tau), \tau]=\eta \mathcal{C}(\lambda \mid \tau),
\end{array}
$$

from which it follows that:

$$
\left[\mathrm{A}(\lambda \mid \tau), \mathrm{S}_{\tau}\right]=\left[\mathrm{D}(\lambda \mid \tau), \mathrm{S}_{\tau}\right]=\left[\mathcal{B}(\lambda \mid \tau), \mathrm{S}_{\tau}\right]=\left[\mathcal{C}(\lambda \mid \tau), \mathrm{S}_{\tau}\right]=0,
$$

and then $\overline{\mathbb{D}}_{(6 \mathrm{VD}), N}^{\mathcal{L} / \mathcal{R}}$ are invariant under the action of these operators; then $\overline{\mathbb{D}}_{(6 \mathrm{VD}), \mathrm{N}}^{\mathcal{L} / \mathcal{R}}$ are invariant also w.r.t. the action of the transfer matrix $\overline{\mathcal{T}}^{(6 \mathrm{VD})}(\lambda \mid \tau)$. Let us now take the trace of (2.41):

$$
\begin{aligned}
& \overline{\mathcal{T}}^{(6 \mathrm{VD})}\left(\lambda_{1} \mid \tau\right) \overline{\mathcal{T}}^{(6 \mathrm{VD})}\left(\lambda_{2} \mid \tau\right)= \\
& =\operatorname{tr}_{12}\left[\overline{\mathcal{M}}_{1}^{(6 \mathrm{VD})}\left(\lambda_{1} \mid \tau\right) \overline{\mathcal{M}}_{2}^{(6 \mathrm{VD})}\left(\lambda_{2} \mid \tau\right)\right] \\
& =\operatorname{tr}_{12}\left[\left(R_{1,2}^{(6 \mathrm{VD})}\left(\lambda_{12} \mid-\tau-\eta \mathrm{S}\right)\right)^{-1} \overline{\mathcal{M}}_{2}^{(6 \mathrm{VD})}\left(\lambda_{2} \mid \tau\right) \overline{\mathcal{M}}_{1}^{(6 \mathrm{VD})}\left(\lambda_{1} \mid \tau\right) R_{1,2}^{(6 \mathrm{VD})}\left(\lambda_{12} \mid \tau\right)\right] \\
& \quad=\operatorname{tr}_{12}\left[\left(R_{1,2}^{(6 \mathrm{VD})}\left(\lambda_{12} \mid-\tau-\eta \mathrm{S}\right)\right)^{-1} \overline{\mathrm{M}}_{2}^{(6 \mathrm{VD})}\left(\lambda_{2} \mid \tau\right) \overline{\mathrm{M}}_{1}^{(6 \mathrm{VD})}\left(\lambda_{1} \mid \tau+\eta \sigma_{2}^{z}\right) \mathrm{T}_{\tau}^{\sigma_{1}^{z}} \mathrm{~T}_{\tau}^{\sigma_{2}^{z}} R_{1,2}^{(6 \mathrm{VD})}\left(\lambda_{12} \mid \tau\right)\right] \\
& =\operatorname{tr}_{12}\left[R_{1,2}^{(6 \mathrm{VD})}\left(\lambda_{12} \mid \tau\right)\left(R_{1,2}^{(6 \mathrm{VD})}\left(\lambda_{12} \mid-\tau-\eta \mathrm{S}\right)\right)^{-1} \overline{\mathrm{M}}_{2}^{(6 \mathrm{VD})}\left(\lambda_{2} \mid \tau\right) \overline{\mathrm{M}}_{1}^{(6 \mathrm{VD})}\left(\lambda_{1} \mid \tau+\eta \sigma_{2}^{z}\right) \mathrm{T}_{\tau}^{\sigma_{1}^{z}} \mathrm{~T}_{\tau}^{\sigma_{2}^{z}}\right]
\end{aligned}
$$

which in $\overline{\mathbb{D}}_{(6 \mathrm{VD}), \mathrm{N}}^{\mathcal{L}}$ coincides with

$$
\operatorname{tr}_{12}\left[\overline{\mathcal{M}}_{2}^{(6 \mathrm{VD})}\left(\lambda_{2} \mid \tau\right) \overline{\mathcal{M}}_{1}^{(6 \mathrm{VD})}\left(\lambda_{1} \mid \tau\right)\right]=\overline{\mathcal{T}}^{(6 \mathrm{VD})}\left(\lambda_{1} \mid \tau\right) \overline{\mathcal{T}}^{(6 \mathrm{VD})}\left(\lambda_{2} \mid \tau\right)
$$

i.e. the commutativity.

From now on we will implicitly assume that $N$ is odd when representations in the linear spaces $\overline{\mathbb{D}}_{(6 \mathrm{VD}), \mathrm{N}}^{\mathcal{L} / \mathcal{R}}$ will be considered.

\subsection{SOV-representations for $\overline{\mathcal{T}}^{(6 \mathrm{VD})}$-spectral problem}

\subsubsection{SOV-representations}

Here, we will show as the standard method to define quantum separation of variable (SOV) representations introduced by Sklyanin [47, 48, 49] for the transfer matrix of 6-vertex Yang-Baxter algebra can be adapted for the dynamical case. In particular, SOV representations for the spectral problem of the antiperiodic $\overline{\mathcal{T}}^{(6 \mathrm{VD})}(\lambda \mid \tau)$ can be defined as the representations where the commutative family of operators $\mathrm{D}(\lambda \mid \tau)$ (or $\mathrm{A}(\lambda \mid \tau)$ ) is pseudo-diagonal and with simple spectrum in the (left/right) $2^{\mathrm{N}}$-dimensional spaces $\overline{\mathbb{D}}_{(6 \mathrm{VD}), \mathrm{N}}^{\mathcal{L}}$. Here, we mean that we can construct explicitly left/right basis of the spaces $\overline{\mathbb{D}}_{(6 \mathrm{VD}), \mathrm{N}}^{\mathcal{L} / \mathcal{R}}$ in terms of pseudo $\mathrm{D}(\lambda \mid \tau)$-eigenstate 22 .

\footnotetext{
${ }^{22}$ What we mean for pseudo-eigenstates will be clarified in the following.
} 
In order to make the construction of these basis some preparation is need; let us define the left and right references states 23 :

$$
\left\langle\mathbf{0}\left|\equiv \otimes_{n=1}^{\mathrm{N}}\left\langle n, h_{n}=0\left|\otimes\left\langle t_{\mathbf{0}}|, \quad| \mathbf{1}\right\rangle \equiv \otimes_{n=1}^{\mathrm{N}}\right| n, h_{n}=1\right\rangle \otimes\right| t_{\mathbf{1}}\right\rangle,
$$

where we are using the notations $\mathbf{0} \equiv\left(h_{1}=0, \ldots, h_{\mathrm{N}}=0\right)$ and $\mathbf{1} \equiv\left(h_{1}=1, \ldots, h_{\mathrm{N}}=1\right)$, so that $t_{\mathbf{0}} \equiv-(\eta \mathbf{N}) / 2$ and $t_{\mathbf{1}} \equiv(\eta \mathrm{N}) / 2$ by the definition (2.43) of $t_{\mathbf{h}}$. Then, we can define the following sets of left and right states:

$$
\left\langle h_{1}, \ldots, h_{\mathrm{N}}\right| \equiv \frac{1}{\mathrm{~N}}\langle\mathbf{0}| \prod_{n=1}^{\mathrm{N}}\left(\frac{\mathcal{C}\left(\xi_{n} \mid \tau\right)}{\mathrm{D}\left(\xi_{n}-\eta\right)}\right)^{h_{n}},
$$

and

$$
\left|h_{1}, \ldots, h_{\mathrm{N}}\right\rangle \equiv \frac{1}{\mathrm{~N}} \prod_{n=1}^{\mathrm{N}}\left(\frac{\mathcal{C}\left(\xi_{n}-\eta \mid \tau\right)}{\mathrm{D}\left(\xi_{n}-\eta\right)}\right)^{\left(1-h_{n}\right)}|\mathbf{1}\rangle,
$$

where $h_{n} \in\{0,1\}, n \in\{1, \ldots, \mathrm{N}\}$. Note that the normalization $\mathrm{N}$ has been introduced to simplify the form of the coupling of the above left and right states. Let us define first the following theta functions with characteristic:

$$
\vartheta_{j}(\lambda)=\sum_{n \in \mathbb{Z}} \exp \left[2 i \pi w \mathrm{~N}\left(n+\frac{1}{2}-\frac{j}{\mathrm{~N}}\right)+2 i \pi \mathrm{N}\left(n+\frac{1}{2}-\frac{j}{\mathrm{~N}}\right)\left(\lambda+\frac{1}{2 \mathrm{~N}}\right)\right],
$$

$\mathrm{N} \in \mathbb{N}$ and $j \in\{0, \ldots, \mathbf{N}-1\}$ which satisfies the periodicity conditions:

$$
\vartheta_{j}(\lambda+1 / \mathrm{N})=-e^{2 \pi i(j / \mathrm{N})} \vartheta_{j}(\lambda), \vartheta_{j}(\lambda+2 w)=-e^{-2 \pi i \mathrm{~N}(w+\lambda)} \vartheta_{j}(\lambda) .
$$

Then we can fix:

$$
\mathrm{N}^{2} \equiv c_{\mathrm{N}} \frac{\left\langle\mathbf{0}\left|\prod_{n=1}^{\mathrm{N}}\left(\frac{\mathcal{C}\left(\xi_{n}-\eta \mid \tau\right)}{\mathrm{D}\left(\xi_{n}-\eta\right)}\right)^{\left(1-h_{n}\right)}\right| \mathbf{1}\right\rangle}{\operatorname{det}_{\mathrm{N}} \Theta_{i j}^{(\mathbf{h} \equiv \mathbf{0})}}
$$

where $c_{\mathrm{N}}$ is a normalization constant defined in $(2.100)$ and $\left\|\Theta_{i j}^{(\mathbf{h})}\right\|$ is the $\mathrm{N} \times \mathrm{N}$ matrix of elements:

$$
\Theta_{i j}^{(\mathbf{h})} \equiv \vartheta_{i-1}\left(\bar{\xi}_{j}^{\left(h_{j}\right)}\right), \quad \bar{\xi}_{a}^{\left(h_{a}\right)}=\xi_{a}^{\left(h_{a}\right)}+\frac{\eta}{2}+\frac{\mathrm{N}-1}{2 \mathrm{~N}}-\frac{1}{\mathrm{~N}} \sum_{a=1}^{\mathrm{N}} \xi_{a}, \quad \xi_{a}^{\left(h_{a}\right)}=\xi_{a}-\eta h_{a} .
$$

It is simple to verify that the states (2.56) and (2.57) are simultaneous (left/right) eigenstates of $\tau$ and $\mathrm{S} \equiv \sum_{a=1}^{\mathrm{N}} \sigma_{a}^{z}$ :

$$
\begin{aligned}
\left\langle h_{1}, \ldots, h_{\mathrm{N}}\right| \tau & =t_{\mathbf{h}}\left\langle h_{1}, \ldots, h_{\mathrm{N}}\right|, \\
\left\langle h_{1}, \ldots, h_{\mathrm{N}}\right| \mathrm{S} & =\mathrm{s}_{\mathbf{h}}\left\langle h_{1}, \ldots, h_{\mathrm{N}}\right|,
\end{aligned}
$$

and:

$$
\begin{aligned}
\tau\left|h_{1}, \ldots, h_{\mathrm{N}}\right\rangle & =\left|h_{1}, \ldots, h_{\mathrm{N}}\right\rangle t_{\mathbf{h}}, \\
\mathrm{S}\left|h_{1}, \ldots, h_{\mathrm{N}}\right\rangle & =\left|h_{1}, \ldots, h_{\mathrm{N}}\right\rangle \mathbf{s}_{\mathbf{h}} .
\end{aligned}
$$

Moreover, under the following condition on the N-tuple of inhomogeneities $\left\{\xi_{1}, \ldots, \xi_{\mathrm{N}}\right\} \in \mathbb{C}^{\mathrm{N}}$ :

$$
\xi_{a} \neq \xi_{b}^{\left(h_{b}\right)} \bmod (2 w) \forall h_{b} \in\{0,1\} \text { and } a<b \in\{1, \ldots, \mathrm{N}\},
$$

the following theorem holds:

\footnotetext{
${ }^{23}$ The left and right states of $\overline{\mathbb{D}}_{(6 \mathrm{VD}), N}^{\mathcal{L} / \mathcal{R}}$ with all spin up and down, respectively.
} 
Theorem 2.2. I) Left SOV-representations Under the condition (2.66), the states (2.56) define a basis of pseudo $\mathrm{D}(\lambda \mid \tau)$-eigenstates in $\overline{\mathbb{D}}_{(6 \mathrm{VD}), \mathrm{N}}^{\mathcal{L}}$; indeed it holds:

$$
\left\langle h_{1}, \ldots, h_{\mathrm{N}}\right| \mathrm{D}(\lambda \mid \tau)=\mathrm{d}_{\boldsymbol{h}}^{\mathcal{L}}(\lambda)\left(\frac{1}{\mathrm{~N}}\langle\boldsymbol{0}| \prod_{n=1}^{\mathrm{N}}\left(\frac{\mathcal{C}\left(\xi_{n} \mid \tau-\eta\right)}{\mathrm{D}\left(\xi_{n}-\eta\right)}\right)^{h_{n}}\right),
$$

where:

$$
\mathrm{d}_{\boldsymbol{h}}^{\mathcal{L}}(\lambda) \equiv \frac{\theta\left(t_{\boldsymbol{o}}-\eta\right)}{\theta\left(t_{\boldsymbol{o}}+\eta\right)} \frac{\theta\left(t_{\boldsymbol{I}}\right)}{\theta\left(t_{\boldsymbol{h}}\right)} \mathrm{d}_{\boldsymbol{h}}(\lambda), \mathrm{d}_{\boldsymbol{h}}(\lambda) \equiv \prod_{n=1}^{\mathrm{N}} \theta\left(\lambda-\xi_{n}^{\left(h_{n}\right)}\right) .
$$

The action of the remaining generators on the generic state $\left\langle h_{1}, \ldots, h_{\mathrm{N}}\right|$ reads:

$$
\begin{aligned}
\left\langle h_{1}, \ldots, h_{\mathrm{N}}\right| \mathcal{C}(\lambda \mid \tau) & =\sum_{a=1}^{\mathrm{N}} \frac{\theta\left(\tau-\lambda+\xi_{a}^{\left(h_{a}\right)}\right)}{\theta(\tau)} \prod_{b \neq a} \frac{\theta\left(\lambda-\xi_{b}^{\left(h_{b}\right)}\right)}{\theta\left(\xi_{a}^{\left(h_{a}\right)}-\xi_{b}^{\left(h_{b}\right)}\right)} \mathrm{D}\left(\xi_{a}^{\left(1-h_{a}\right)}\right)\left\langle h_{1}, \ldots, h_{\mathrm{N}}\right| \mathrm{T}_{a}^{+}, \\
\left\langle h_{1}, \ldots, h_{\mathrm{N}}\right| \mathcal{B}(\lambda \mid \tau) & =\sum_{a=1}^{\mathrm{N}} \frac{\theta\left(\tau-\lambda+\xi_{a}^{\left(h_{a}\right)}\right)}{\theta(\tau)} \prod_{b \neq a} \frac{\theta\left(\lambda-\xi_{b}^{\left(h_{b}\right)}\right)}{\theta\left(\xi_{a}^{\left(h_{a}\right)}-\xi_{b}^{\left(h_{b}\right)}\right)} \mathrm{A}\left(\xi_{a}^{\left(1-h_{a}\right)}\right)\left\langle h_{1}, \ldots, h_{\mathrm{N}}\right| \mathrm{T}_{a}^{-},
\end{aligned}
$$

where:

$$
\left\langle h_{1}, \ldots, h_{\mathrm{N}}\right| \mathrm{T}_{a}^{ \pm}=\left\langle h_{1}, \ldots, h_{a} \pm 1, \ldots, h_{\mathrm{N}}\right|,
$$

and $\mathrm{A}(\lambda \mid \tau)$ is uniquely defined by the quantum determinant relation.

II) Right SOV-representations Under the condition (2.66), the states (2.57) define a basis of pseudo $\mathrm{D}(\lambda \mid \tau)$ eigenstates in $\overline{\mathbb{D}}_{(6 \mathrm{VD}), \mathrm{N}}^{\mathcal{R}}$; indeed it holds:

$$
\mathrm{D}(\lambda \mid \tau+\eta)\left|h_{1}, \ldots, h_{\mathrm{N}}\right\rangle=\left(\frac{1}{\mathrm{~N}} \prod_{n=1}^{\mathrm{N}}\left(\frac{\mathcal{C}\left(\xi_{n}-\eta \mid \tau+\eta\right)}{\mathrm{D}\left(\xi_{n}-\eta\right)}\right)^{\left(1-h_{n}\right)}|\boldsymbol{I}\rangle\right) \mathrm{d}_{\boldsymbol{h}}^{\mathcal{R}}(\lambda),
$$

where:

$$
\mathrm{d}_{\boldsymbol{h}}^{\mathcal{R}}(\lambda) \equiv \frac{\theta\left(t_{\boldsymbol{h}}+\eta\right)}{\theta\left(t_{\boldsymbol{I}}+\eta\right)} \mathrm{d}_{\boldsymbol{h}}(\lambda) .
$$

The action of the remaining generators on the generic state $\left|h_{1}, \ldots, h_{\mathrm{N}}\right\rangle$ reads:

$$
\begin{aligned}
& \mathcal{C}(\lambda \mid \tau)\left|h_{1}, \ldots, h_{\mathrm{N}}\right\rangle=\sum_{a=1}^{\mathrm{N}} \mathrm{T}_{a}^{-}\left|h_{1}, \ldots, h_{\mathrm{N}}\right\rangle \frac{\theta\left(\tau-\lambda+\xi_{a}^{\left(h_{a}\right)}\right)}{\theta(\tau)} \prod_{b \neq a} \frac{\theta\left(\lambda-\xi_{b}^{\left(h_{b}\right)}\right)}{\theta\left(\xi_{a}^{\left(h_{a}\right)}-\xi_{b}^{\left(h_{b}\right)}\right)} \mathrm{D}\left(\xi_{a}^{\left(h_{a}\right)}\right), \\
& \mathcal{B}(\lambda \mid \tau)\left|h_{1}, \ldots, h_{\mathrm{N}}\right\rangle=\sum_{a=1}^{\mathrm{N}} \mathrm{T}_{a}^{+}\left|h_{1}, \ldots, h_{\mathrm{N}}\right\rangle \frac{\theta\left(\tau-\lambda+\xi_{a}^{\left(h_{a}\right)}\right)}{\theta(\tau)} \prod_{b \neq a} \frac{\theta\left(\lambda-\xi_{b}^{\left(h_{b}\right)}\right)}{\theta\left(\xi_{a}^{\left(h_{a}\right)}-\xi_{b}^{\left(h_{b}\right)}\right)} \mathrm{A}\left(\xi_{a}^{\left(h_{a}\right)}\right),
\end{aligned}
$$

where:

$$
\mathrm{T}_{a}^{ \pm}\left|h_{1}, \ldots, h_{\mathrm{N}}\right\rangle=\left|h_{1}, \ldots, h_{a} \pm 1, \ldots, h_{\mathrm{N}}\right\rangle,
$$

and $\mathrm{A}(\lambda \mid \tau)$ is uniquely defined by the quantum determinant relation. 
Proof. The proof of the theorem is based on the dynamical Yang-Baxter commutation relations and on the fact that the left and right references states are $\mathrm{D}(\lambda \mid \tau)$-eigenstates:

$$
\langle\mathbf{0}| \mathrm{A}(\lambda \mid \tau)=\mathrm{A}(\lambda)\langle\mathbf{0}|, \quad\langle\mathbf{0}| \mathrm{D}(\lambda \mid \tau)=\mathrm{D}\left(\lambda \mid t_{\mathbf{0}}\right)\langle\mathbf{0}|, \quad\langle\mathbf{0}| \mathcal{B}(\lambda \mid \tau)=\underline{0}, \quad\langle\mathbf{0}| \mathcal{C}(\lambda \mid \tau) \neq \underline{0},
$$

and similarly:

$$
\mathrm{D}(\lambda \mid \tau)|\mathbf{1}\rangle=|\mathbf{1}\rangle \mathrm{A}(\lambda), \quad \mathrm{A}(\lambda \mid \tau)|\mathbf{1}\rangle=|\mathbf{1}\rangle \mathrm{D}\left(\lambda \mid t_{\mathbf{0}}\right), \quad \mathcal{B}(\lambda \mid \tau)|\mathbf{1}\rangle=\underline{0}, \quad \mathcal{C}(\lambda \mid \tau)|\mathbf{1}\rangle \neq \underline{0},
$$

where:

$$
\mathrm{D}\left(\lambda \mid t_{\mathbf{0}}\right) \equiv \mathrm{D}(\lambda) \frac{\theta\left(\eta-t_{\mathbf{0}}\right)}{\theta\left(\eta+t_{\mathbf{0}}\right)} .
$$

Indeed, to prove that (2.56) and (2.57) are left and right pseudo-eigenstates of $\mathrm{D}(\lambda \mid \tau)$ as stated in (2.67) and (2.72), we have just to repeat the standard computations in algebraic Bethe ansatz [14] as done in [3]. More in detail, considering the action of $\mathcal{D}(\lambda \mid \tau)$ on the left states $\left\langle h_{1}, \ldots, h_{\mathrm{N}}\right|$ and following the steps given in the proof of Theorem 3.2 of [3] by using here the dynamical 6-vertex commutation relation:

$$
\begin{aligned}
\mathcal{C}(\mu \mid \tau) \mathcal{D}(\lambda \mid \tau) & =[\mathcal{D}(\lambda \mid \tau) \mathcal{C}(\mu \mid \tau) \theta(\lambda-\mu+\eta) \theta(\tau)-\mathcal{D}(\mu \mid \tau) \mathcal{C}(\lambda \mid \tau) \theta(\eta) \theta(\tau+\lambda-\mu)] \\
& \times \frac{1}{\theta(\lambda-\mu) \theta(\tau+\eta)},
\end{aligned}
$$

we get:

$$
\left\langle h_{1}, \ldots, h_{\mathrm{N}}\right| \mathcal{D}(\lambda \mid \tau)=\mathrm{d}_{\mathbf{h}}^{\mathcal{L}}(\lambda)\left[\frac{1}{\mathrm{~N}}\left(\langle\mathbf{0}| \mathrm{T}_{\tau}^{-}\right) \prod_{n=1}^{\mathrm{N}}\left(\frac{\mathcal{C}\left(\xi_{n} \mid \tau\right)}{\mathrm{D}\left(\xi_{n}-\eta\right)}\right)^{h_{n}}\right] .
$$

Similarly, by using the dynamical 6-vertex commutation relation:

$$
\begin{aligned}
\mathcal{D}(\lambda \mid \tau) \mathcal{C}(\mu \mid \tau) & =\frac{1}{\theta(\mu-\lambda) \theta(\tau+\eta)}[\theta(\mu-\lambda+\eta) \theta(\tau) \mathcal{C}(\mu \mid \tau) \mathcal{D}(\lambda \mid \tau) \\
& -\theta(\eta) \theta(\tau+\mu-\lambda) \mathcal{C}(\lambda \mid \tau) \mathcal{D}(\mu \mid \tau)
\end{aligned}
$$

we get:

$$
\mathcal{D}(\lambda \mid \tau)\left|h_{1}, \ldots, h_{\mathrm{N}}\right\rangle=\left(\frac{1}{\mathrm{~N}} \prod_{n=1}^{\mathrm{N}}\left(\frac{\mathcal{C}\left(\xi_{n}-\eta \mid \tau\right)}{\mathrm{D}\left(\xi_{n}-\eta\right)}\right)^{\left(1-h_{n}\right)}\left(\mathrm{T}_{\tau}^{-}|\mathbf{1}\rangle\right)\right) \mathrm{d}_{\mathbf{h}}^{\mathcal{L}}(\lambda) .
$$

From the formulae (2.81) and (2.83) by using the commutation relations (2.1), $\mathrm{D}(\lambda \mid \tau)=\mathcal{D}(\lambda \mid \tau) \mathrm{T}_{\tau}^{+}$and $\mathrm{D}(\lambda \mid \tau+$ $\eta)=\mathrm{T}_{\tau}^{+} \mathcal{D}(\lambda \mid \tau)$ then the formulae (2.67) and (2.72) simply follow.

Let us prove now that the states $\left\langle h_{1}, \ldots, h_{\mathrm{N}}\right|$ form a set of $2^{\mathrm{N}}$ independent states, i.e. a basis of $\overline{\mathbb{D}}_{(6 \mathrm{VD}), \mathrm{N}}^{\mathcal{L}}$; similarly, we can prove that the states $\left|h_{1}, \ldots, h_{\mathrm{N}}\right\rangle$ form a basis of $\overline{\mathbb{D}}_{(6 \mathrm{VD}), \mathrm{N}}^{\mathcal{R}}$. By definition we have to prove that their linear combination to zero:

$$
\sum_{h_{1}, \ldots, h_{\mathrm{N}}=0}^{1} c_{\mathbf{h}}\left\langle h_{1}, \ldots, h_{\mathrm{N}}\right|=\underline{0}
$$

holds only if all the coefficients are zeros. Let us denote with $\overline{\mathbf{h}}=\left\{\bar{h}_{1}, \ldots, \bar{h}_{\mathrm{N}}\right\}$ the generic N-tuple in $\{0,1\}^{\mathrm{N}}$ then by applying to both side of (2.84) the operator product:

$$
\prod_{n=1}^{\mathrm{N}} \mathcal{D}\left(\xi_{n}^{\left(\bar{k}_{n}\right)} \mid \tau\right) \text { with } \bar{k}_{n}=\bar{h}_{n}+1 \bmod 2 \in\{0,1\}
$$


we get:

$$
c_{\overline{\mathbf{h}}} \prod_{n=1}^{\mathrm{N}} \mathrm{d}_{\overline{\mathbf{h}}}^{\mathcal{L}}\left(\xi_{n}^{\left(\bar{k}_{n}\right)}\right)\left[\left(\langle\mathbf{0}| \mathrm{T}_{\tau}^{-\mathrm{N}}\right) \prod_{n=1}^{\mathrm{N}}\left(\frac{\mathcal{C}\left(\xi_{n} \mid \tau\right)}{\mathrm{D}\left(\xi_{n}-\eta\right)}\right)^{\bar{h}_{n}}\right]=\underline{0}
$$

which implies $c_{\overline{\mathbf{h}}}=0$ being:

$$
\prod_{n=1}^{\mathrm{N}} \mathrm{d}_{\overline{\mathbf{h}}}\left(_{n}^{\left(\bar{k}_{n}\right)}\right) \neq 0, \quad\left[\left(\langle\mathbf{0}| \mathrm{T}_{\tau}^{-\mathrm{N}}\right) \prod_{n=1}^{\mathrm{N}}\left(\frac{\mathcal{C}\left(\xi_{n} \mid \tau\right)}{\mathrm{D}\left(\xi_{n}-\eta\right)}\right)^{\bar{h}_{n}}\right] \neq \underline{0} .
$$

The action of $\mathcal{B}\left(\xi_{n}^{\left(h_{n}\right)} \mid \tau\right)$ and $\mathcal{C}\left(\xi_{n}^{\left(h_{n}\right)} \mid \tau\right)$ on the left and right states (2.56) and (2.57) follows by imposing the dynamical Yang-Baxter commutation relations and the quantum determinant relations:

$$
\left\langle h_{1}, \ldots, h_{\mathrm{N}}\left|\operatorname{det}_{q} \mathrm{M}(\lambda)=\mathrm{A}(\lambda) \mathrm{D}(\lambda-\eta)\left\langle h_{1}, \ldots, h_{\mathrm{N}}\left|, \quad \operatorname{det}_{q} \mathrm{M}(\lambda)\right| h_{1}, \ldots, h_{\mathrm{N}}\right\rangle=\mathrm{A}(\lambda) \mathrm{D}(\lambda-\eta)\right| h_{1}, \ldots, h_{\mathrm{N}}\right\rangle,
$$

where we have used that:

$$
\left.\frac{\theta(\tau+\eta \mathrm{S})}{\theta(\tau)}\right|_{\left\langle h_{1}, \ldots, h_{\mathrm{N}}|,| h_{1}, \ldots, h_{\mathrm{N}}\right\rangle}=\frac{\theta\left(-t_{\mathbf{h}}\right)}{\theta\left(t_{\mathbf{h}}\right)}=-1,
$$

and in the quantum determinant $\operatorname{det}_{q} \mathrm{M}(\lambda)$ we use the identities:

$$
\begin{aligned}
& \mathcal{A}(\lambda \mid \tau) \mathcal{D}(\lambda-\eta \mid \tau)-\mathcal{B}(\lambda \mid \tau) \mathcal{C}(\lambda-\eta \mid \tau)=\mathrm{A}(\lambda \mid \tau) \mathrm{D}(\lambda-\eta \mid \tau+\eta)-\mathrm{B}(\lambda \mid \tau) \mathrm{C}(\lambda-\eta \mid \tau-\eta), \\
& \mathcal{D}(\lambda \mid \tau) \mathcal{A}(\lambda-\eta \mid \tau)-\mathcal{C}(\lambda \mid \tau) \mathcal{B}(\lambda-\eta \mid \tau)=\mathrm{D}(\lambda \mid \tau) \mathrm{A}(\lambda-\eta \mid \tau-\eta)-\mathrm{C}(\lambda \mid \tau) \mathrm{B}(\lambda-\eta \mid \tau+\eta)
\end{aligned}
$$

Finally, the left (2.69)-(2.70) and right (2.74)-(2.75) representations of $\mathcal{B}(\lambda \mid \tau)$ and $\mathcal{C}(\lambda \mid \tau)$ are just interpolation formulae in the special points $\left\{\xi_{1}^{\left(h_{1}\right)}, \ldots, \xi_{\mathrm{N}}^{\left(h_{N}\right)}\right\}$ which hold for elliptic polynomials as illustrated for example in Appendix A of [159].

\subsubsection{SOV-decomposition of the identity}

The previous results allow to write the following spectral decomposition of the identity $\mathbb{I}$ :

$$
\mathbb{I} \equiv \sum_{h_{1}, \ldots, h_{\mathrm{N}}=0}^{1} \mu_{\mathbf{h}}\left|h_{1}, \ldots, h_{\mathrm{N}}\right\rangle\left\langle h_{1}, \ldots, h_{\mathrm{N}}\right|,
$$

in terms of the left and right SOV-basis. Here,

$$
\mu_{\mathbf{h}} \equiv \frac{1}{\left\langle h_{1}, \ldots, h_{\mathrm{N}} \mid h_{1}, \ldots, h_{\mathrm{N}}\right\rangle},
$$

is the analogous of the so-called Sklyanin's measure; which is discrete in these representations and defined by the following proposition:

Proposition 2.2. Let $\left\langle h_{1}, \ldots, h_{N}\right|$ be the generic covector (2.56) and $\left|k_{1}, \ldots, k_{N}\right\rangle$ be the generic vector (2.57), then it holds:

$$
\left\langle h_{1}, \ldots, h_{\mathrm{N}} \mid k_{1}, \ldots, k_{\mathrm{N}}\right\rangle=\frac{\prod_{c=1}^{\mathrm{N}} \delta_{h_{c}, k_{c}}}{\operatorname{det}_{\mathrm{N}} \Theta_{i j}^{(\boldsymbol{h})}}
$$

where $\Theta_{i j}^{(\boldsymbol{h})}$ is the $\mathrm{N} \times \mathrm{N}$ matrix defined in (2.61). Then, the SOV-decomposition of the identity explicitly reads:

$$
\mathbb{I} \equiv \sum_{h_{1}, \ldots, h_{\mathrm{N}}=0}^{1} \operatorname{det}_{\mathrm{N}} \Theta_{i j}^{(\boldsymbol{h})}\left|h_{1}, \ldots, h_{\mathrm{N}}\right\rangle\left\langle h_{1}, \ldots, h_{\mathrm{N}}\right| .
$$


Proof. The fact that $\left\langle h_{1}, \ldots, h_{\mathrm{N}}\right|$ and $\left|k_{1}, \ldots, k_{\mathrm{N}}\right\rangle$ are simultaneous eigenstates of $\tau$ with eigenvalues $t_{\mathbf{h}}$ and $t_{\mathbf{k}}$, respectively, implies that the 1.h.s. of (2.94) is zero unless:

$$
\sum_{c=1}^{\mathrm{N}} h_{c}=\sum_{c=1}^{\mathrm{N}} k_{c}
$$

Let us assume now that $\mathbf{h} \neq \mathbf{k}$ but that they satisfy the condition (2.96). Under these conditions it is easy to understand that there exists at least one $n \in\{1, \ldots, \mathrm{N}\}$ such that $h_{n}=1$ and $k_{n}=0$ and then the 1.h.s. of (2.94) contains the product of operators $\mathcal{C}\left(\xi_{n} \mid \tau\right) \mathcal{C}\left(\xi_{n}-\eta \mid \tau\right)$ which is zero for the standard 6-vertex annihilation identities. Then, as stated in (2.94), $\left\langle h_{1}, \ldots, h_{\mathrm{N}} \mid k_{1}, \ldots, k_{\mathrm{N}}\right\rangle$ is zero for $\mathbf{h} \neq \mathbf{k}$; so we are left with the computations for $\mathbf{h}=\mathbf{k}$. In order to compute them, let us compute the matrix elements:

$$
x_{a} \equiv\left\langle h_{1}, \ldots, h_{a}=0, \ldots, h_{\mathrm{N}}\left|\mathcal{C}\left(\xi_{a} \mid \tau\right)\right| h_{1}, \ldots, h_{a}=1, \ldots, h_{\mathrm{N}}\right\rangle,
$$

where $a \in\{1, \ldots, \mathrm{N}\}$. Then using the left action of the operator $\mathcal{C}\left(\eta_{a} \mid \tau\right)$ we get:

$$
x_{a}=\mathrm{D}\left(\xi_{a}-\eta\right)\left\langle h_{1}, \ldots, h_{a}=1, \ldots, h_{\mathrm{N}} \mid h_{1}, \ldots, h_{a}=1, \ldots, h_{\mathrm{N}}\right\rangle,
$$

while using the right action of the operator $\mathcal{C}\left(\eta_{a} \mid \tau\right)$ and the orthogonality of right and left pseudo D-eigenstates corresponding to different eigenvalues we get:

$$
\begin{aligned}
x_{a}= & \prod_{b \neq a} \frac{\theta\left(\xi_{a}-\xi_{b}+\eta h_{b}\right)}{\theta\left(\xi_{a}-\eta-\xi_{b}+\eta h_{b}\right)} \mathrm{D}\left(\xi_{a}-\eta\right)\left\langle h_{1}, \ldots, h_{a}=0, \ldots, h_{\mathrm{N}} \mid h_{1}, \ldots, h_{a}=0, \ldots, h_{\mathrm{N}}\right\rangle \\
& \times \frac{\theta\left(t_{\mathbf{h}\left(h_{a}=1\right)}-\eta\right)}{\theta\left(t_{\mathbf{h}\left(h_{a}=1\right)}\right)}
\end{aligned}
$$

and so:

$$
\frac{\left\langle h_{1}, \ldots, h_{a}=1, \ldots, h_{\mathrm{N}} \mid h_{1}, \ldots, h_{a}=1, \ldots, h_{\mathrm{N}}\right\rangle}{\left\langle h_{1}, \ldots, h_{a}=0, \ldots, h_{\mathrm{N}} \mid h_{1}, \ldots, h_{a}=0, \ldots, h_{\mathrm{N}}\right\rangle}=\frac{\theta\left(t_{\mathbf{h}\left(h_{a}=0\right)}\right)}{\theta\left(t_{\mathbf{h}\left(h_{a}=1\right)}\right)} \prod_{b \neq a, b=1}^{\mathrm{N}} \frac{\theta\left(\xi_{a}^{(0)}-\xi_{b}^{\left(h_{b}\right)}\right)}{\theta\left(\xi_{a}^{(1)}-\xi_{b}^{\left(h_{b}\right)}\right)}
$$

from which the proposition simply follows when we use the identity 24 :

$$
\operatorname{det}_{\mathrm{N}} \Theta_{i j}^{(\mathbf{h})}=c_{\mathrm{N}} \theta\left(\sum_{a=1}^{\mathrm{N}} \bar{\xi}_{a}^{\left(h_{a}\right)}-\frac{\mathrm{N}-1}{2}\right) \prod_{1 \leq a<b \leq \mathrm{N}} \theta\left(\xi_{a}^{\left(h_{a}\right)}-\xi_{b}^{\left(h_{b}\right)}\right),
$$

and we recall that:

$$
t_{\mathbf{h}}=-\sum_{a=1}^{\mathrm{N}} \bar{\xi}_{a}^{\left(h_{a}\right)}+\frac{\mathrm{N}-1}{2}
$$

and that the choice of the normalization $\mathrm{N}$ implies:

$$
\langle\mathbf{0} \mid \mathbf{0}\rangle=\frac{1}{\operatorname{det}_{N} \Theta_{i j}^{(\mathbf{0})}} .
$$

\footnotetext{
${ }^{24}$ See for example Proposition 4 of [159] for a proof of it.
} 


\subsection{SOV characterization of $\overline{\mathcal{T}}^{(6 \mathrm{VD})}$-spectrum}

Let us denote with $\Sigma_{\overline{\mathcal{T}}^{(6 \mathrm{VD})}}$ the set of the eigenvalue functions $\mathrm{t}_{6 \mathrm{VD}}(\lambda)$ of the transfer matrix $\overline{\mathcal{T}}^{(6 \mathrm{VD})}(\lambda \mid \tau)$, then the following characterization of the $\overline{\mathcal{T}}^{(6 \mathrm{VD})}$-spectrum (eigenvalues \& eigenstates) in quantum separation of variables holds:

Theorem 2.3. For any fixed $\mathrm{N}$-tuple of inhomogeneities $\left\{\xi_{1}, \ldots, \xi_{\mathrm{N}}\right\} \in \mathbb{C}^{\mathrm{N}}$ satisfying (2.66) the spectrum of $\overline{\mathcal{T}}^{(6 \mathrm{VD})}(\lambda \mid \tau)$ in $\overline{\mathbb{D}}_{(6 \mathrm{VD}), \mathrm{N}}^{\mathcal{L} / \mathcal{R}}$ is simple and $\Sigma_{\overline{\mathcal{T}}^{(6 \mathrm{VD})}}$ coincides with the set of functions of the form:

$$
\mathrm{t}_{6 \mathrm{VD}}(\lambda)=\sum_{a=1}^{\mathrm{N}} \frac{\theta\left(t_{\boldsymbol{o}}-\lambda+\xi_{a}\right)}{\theta\left(t_{\boldsymbol{o}}\right)} \prod_{b \neq a} \frac{\theta\left(\lambda-\xi_{b}\right)}{\theta\left(\xi_{a}-\xi_{b}\right)} \mathrm{t}_{6 \mathrm{VD}}\left(\xi_{a}\right)
$$

which are solutions of the discrete system of equations:

$$
\mathrm{t}_{6 \mathrm{VD}}\left(\xi_{a}^{(0)}\right) \mathrm{t}_{6 \mathrm{VD}}\left(\xi_{a}^{(1)}\right)=\mathrm{A}\left(\xi_{a}^{(0)}\right) \mathrm{D}\left(\xi_{a}^{(1)}\right), \quad \forall a \in\{1, \ldots, \mathrm{N}\} .
$$

I) The right $\overline{\mathcal{T}}^{(6 \mathrm{VD})}$-eigenstate corresponding to $\mathrm{t}_{6 \mathrm{VD}}(\lambda) \in \Sigma_{\overline{\mathcal{T}}^{(6 \mathrm{VD})}}$ is characterized by:

$$
\left|\mathrm{t}_{6 \mathrm{VD}}\right\rangle=\sum_{h_{1}, \ldots, h_{\mathrm{N}}=0}^{1} \prod_{a=1}^{\mathrm{N}} Q_{\mathrm{t}}\left(\xi_{a}^{\left(h_{a}\right)}\right) \operatorname{det}_{\mathrm{N}} \Theta_{i j}^{(\boldsymbol{h})}\left|h_{1}, \ldots, h_{\mathrm{N}}\right\rangle,
$$

up to an overall normalization, where the coefficients are characterized by:

$$
Q_{\mathrm{t}}\left(\xi_{a}^{(1)}\right) / Q_{\mathrm{t}}\left(\xi_{a}^{(0)}\right)=\mathrm{t}_{6 V D}\left(\xi_{a}^{(0)}\right) / \mathrm{D}\left(\xi_{a}^{(1)}\right)
$$

II) The left $\overline{\mathcal{T}}^{(6 \mathrm{VD})}$-eigenstate corresponding to $\mathrm{t}_{6 \mathrm{VD}}(\lambda) \in \Sigma_{\overline{\mathcal{T}}^{(6 \mathrm{VD})}}$ is characterized by:

$$
\left\langle\mathrm{t}_{6 \mathrm{VD}}\right|=\sum_{h_{1}, \ldots, h_{\mathrm{N}}=0}^{1} \prod_{a=1}^{\mathrm{N}} \bar{Q}_{\mathrm{t}}\left(\xi_{a}^{\left(h_{a}\right)}\right) \operatorname{det}_{\mathrm{N}} \Theta_{i j}^{(\boldsymbol{h})}\left\langle h_{1}, \ldots, h_{\mathrm{N}}\right|,
$$

up to an overall normalization, where the coefficients are characterized by:

$$
\bar{Q}_{\mathrm{t}}\left(\xi_{a}^{(1)}\right) / \bar{Q}_{\mathrm{t}}\left(\xi_{a}^{(0)}\right)=\mathrm{t}_{6 \mathrm{VD}}\left(\xi_{a}^{(0)}\right) / \mathrm{A}\left(\xi_{a}^{(0)}\right) .
$$

Proof. Let $\left\langle\mathrm{t}_{6 \mathrm{VD}}\right|$ be a $\overline{\mathcal{T}}^{(6 \mathrm{VD})}$-eigenstate corresponding to the $\overline{\mathcal{T}}^{(6 \mathrm{VD})}$-eigenvalue $\mathrm{t}_{6 \mathrm{VD}}(\lambda)$, then the coefficients (wave-functions):

$$
\Psi_{t}(\mathbf{h}) \equiv\left\langle\mathrm{t}_{6 \mathrm{VD}} \mid h_{1}, \ldots, h_{\mathrm{N}}\right\rangle
$$

of $\left\langle\mathrm{t}_{6 \mathrm{VD}}\right|$ in the right SOV-basis satisfy the equations:

$$
\mathrm{t}_{6 \mathrm{VD}}\left(\xi_{n}^{\left(h_{n}\right)}\right) \Psi_{t}(\mathbf{h})=\mathrm{A}\left(\xi_{n}^{\left(h_{n}\right)}\right) \Psi_{t}\left(\mathrm{~T}_{n}^{+}(\mathbf{h})\right)+\mathrm{D}\left(\xi_{n}^{\left(h_{n}\right)}\right) \Psi_{t}\left(\mathbf{T}_{n}^{-}(\mathbf{h})\right),
$$

for any $n \in\{1, \ldots, \mathbf{N}\}$ and $\mathbf{h} \in\{0,1\}^{\mathrm{N}}$, where we have denoted:

$$
\mathbf{T}_{n}^{ \pm}(\mathbf{h}) \equiv\left(h_{1}, \ldots, h_{n} \pm 1, \ldots, h_{\mathrm{N}}\right) .
$$

These equations are obtained by computing the matrix elements:

$$
\left\langle\mathrm{t}_{6 \mathrm{VD}}\left|\overline{\mathcal{T}}^{(6 \mathrm{VD})}\left(\xi_{n}^{\left(h_{n}\right)} \mid \tau\right)\right| h_{1}, \ldots, h_{\mathrm{N}}\right\rangle .
$$


In particular, acting with $\overline{\mathcal{T}}^{(6 \mathrm{VD})}\left(\xi_{n}^{\left(h_{n}\right)} \mid \tau\right)$ on $\left\langle\mathrm{t}_{6 \mathrm{VD}}\right|$ the 1.h.s. of (2.110) is reproduced while acting on $\left|h_{1}, \ldots, h_{\mathrm{N}}\right\rangle$ by using the SOV representation of $\overline{\mathcal{T}}^{(6 \mathrm{VD})}\left(\xi_{n}^{\left(h_{n}\right)} \mid \tau\right)$ the r.h.s. of (2.110) is reproduced. Moreover, the representation (2.103) for the $\overline{\mathcal{T}}^{(6 \mathrm{VD})}$-eigenvalue functions $\mathrm{t}_{6 \mathrm{VD}}(\lambda)$ follows by computing the matrix element:

$$
\left\langle\mathrm{t}_{6 \mathrm{VD}}\left|\overline{\mathcal{T}}^{(6 \mathrm{VD})}(\lambda \mid \tau)\right| \mathbf{0}\right\rangle
$$

and by using (2.110) to rewrite the r.h.s. in the desired form.

Then in the SOV representations the spectral problem for $\overline{\mathcal{T}}^{(6 \mathrm{VD})}(\lambda \mid \tau)$ is reduced to a discrete system of $2^{\mathrm{N}}$ Baxterlike equations (2.110) in the class of function of the form (2.103). Taking into account the identities:

$$
\mathrm{A}\left(\xi_{n}^{(1)}\right)=\mathrm{D}\left(\xi_{n}^{(0)}\right)=0
$$

this system coincides with a system of homogeneous equations:

$$
\left(\begin{array}{cc}
\mathrm{t}_{6 \mathrm{VD}}\left(\xi_{n}^{(0)}\right) & -\mathrm{A}\left(\xi_{n}^{(0)}\right) \\
-\mathrm{D}\left(\xi_{n}^{(1)}\right) & \mathrm{t}_{6 \mathrm{VD}}\left(\xi_{n}^{(1)}\right)
\end{array}\right)\left(\begin{array}{c}
\Psi_{t}\left(h_{1}, \ldots, h_{n}=0, \ldots, h_{1}\right) \\
\Psi_{t}\left(h_{1}, \ldots, h_{n}=1, \ldots, h_{1}\right)
\end{array}\right)=\left(\begin{array}{c}
0 \\
0
\end{array}\right)
$$

for any $n \in\{1, \ldots, N\}$ with $h_{m \neq n} \in\{0,1\}$. The condition $\mathrm{t}_{6 \mathrm{VD}}(\lambda) \in \Sigma_{\overline{\mathcal{T}}^{(6 \mathrm{VD})}}$ is then equivalent to the requirement that the determinants of the $2 \times 2$ matrices in (2.115) must be zero for any $n \in\{1, \ldots, \mathrm{N}\}$, i.e. the equation (2.104). On the other hand being:

$$
\mathrm{A}\left(\xi_{n}^{(0)}\right) \neq 0 \text { and } \mathrm{D}\left(\xi_{n}^{(1)}\right) \neq 0
$$

the rank of the matrices in (2.115) is 1 and then up to an overall normalization the solution is unique:

$$
\frac{\Psi_{t}\left(h_{1}, \ldots, h_{n}=1, \ldots, h_{1}\right)}{\Psi_{t}\left(h_{1}, \ldots, h_{n}=0, \ldots, h_{1}\right)}=\frac{\mathrm{t}_{6 \mathrm{VD}}\left(\xi_{n}^{(0)}\right)}{\mathrm{A}\left(\xi_{n}^{(0)}\right)}
$$

for any $n \in\{1, \ldots, N\}$ with $h_{m \neq n} \in\{0,1\}$. This implies that given a $\mathrm{t}_{6 \mathrm{VD}}(\lambda) \in \Sigma_{\overline{\mathcal{T}}^{(6 \mathrm{VD})}}$ there exist (up to normalization) one and only one corresponding $\overline{\mathcal{T}}^{(6 \mathrm{VD})}$-eigenstate $\left\langle\mathrm{t}_{6 \mathrm{VD}}\right|$ with coefficients which have the factorized form given in (2.107)-(2.108) and then the $\overline{\mathcal{T}}^{(6 \mathrm{VD})}$-spectrum is simple. The proof for the right eigenstates is given in a similar way.

Let us remark that the previous theorem completely characterize the spectrum of the transfer matrix $\overline{\mathcal{T}}^{(6 \mathrm{VD})}(\lambda \mid \tau)$ in $\overline{\mathbb{D}}_{(6 \mathrm{VD}), \mathrm{N}}^{\mathcal{L} / \mathcal{R}}$. However, a reformulation of the SOV characterization of the $\overline{\mathcal{T}}^{(6 \mathrm{VD})}$-spectrum by functional equations can be important for practical aims. One standard way to accomplish this result is by the construction of a Baxter Q-operator whose functional equation reduces to the finite system of Baxter-like equations (2.110) when computed in the eigenvalues of the quantum separate variables, i.e. the operators zero of $D(\lambda \mid \tau)$. This construction is currently under analysis and it can be developed along the same lines presented in [73] for the antiperiodic XXZ spin-1/2 chain for general values of the coupling $\eta$. For the elliptic roots of unit case, we can construct the functional equation directly by using cyclic representations for the operator $\tau$; these interesting issues will be developed in a forthcoming paper. Finally, let us remark that once the Q-operator is constructed its eigenvalues can be used to completely characterize not only the eigenvalues but also the eigenstates of the antiperiodic dynamical 6-vertex transfer matrix in the SOV framework. Indeed, it is enough to introduce in formula (2.105) for the $Q_{\mathbf{t}}\left(\xi_{a}^{\left(h_{a}\right)}\right)$ the eigenvalue of the Q-operator computed in $\xi_{a}^{\left(h_{a}\right)}$ to get the corresponding simultaneous eigenstate. 


\subsection{Action of left separate states on right separate states}

As for the others quantum integrable models analyzed by SOV in [1]-[5], a special role is played by the left and right separate states in the SOV representations. These are states which have factorized coefficients in the SOV representations similar to those of the transfer matrix eigenstates; more in detail we say that a covector $\langle\alpha| \in$ $\overline{\mathbb{D}}_{(6 \mathrm{VD}), \mathrm{N}}^{\mathcal{L}}$ and a vector $|\beta\rangle \in \overline{\mathbb{D}}_{(6 \mathrm{VD}), \mathrm{N}}^{\mathcal{R}}$ are separate states if they admit the following SOV-decompositions:

$$
\begin{aligned}
& \langle\alpha|=\sum_{h_{1}, \ldots, h_{\mathrm{N}}=0}^{1} \prod_{a=1}^{\mathrm{N}} \alpha_{a}\left(\xi_{a}^{\left(h_{a}\right)}\right) \operatorname{det}_{\mathrm{N}} \Theta_{i j}^{(\mathbf{h})}\left\langle h_{1}, \ldots, h_{\mathrm{N}}\right|, \\
& |\beta\rangle=\sum_{h_{1}, \ldots, h_{\mathrm{N}}=0}^{1} \prod_{a=1}^{\mathrm{N}} \beta_{a}\left(\xi_{a}^{\left(h_{a}\right)}\right) \operatorname{det}_{\mathrm{N}} \Theta_{i j}^{(\mathbf{h})}\left|h_{1}, \ldots, h_{\mathrm{N}}\right\rangle .
\end{aligned}
$$

The main interest toward these states is the simple determinant form for the action of left separate states on the right ones characterized by the following:

Proposition 2.3. The left $\langle\alpha|$ and the right $|\beta\rangle$ separate states satisfy the identities:

$$
\langle\alpha \mid \beta\rangle=\operatorname{det}_{\mathrm{N}}\left\|\mathcal{F}_{a, b}^{(\alpha, \beta)}\right\| \text { with } \mathcal{F}_{a, b}^{(\alpha, \beta)} \equiv \sum_{h=0}^{1} \alpha_{a}\left(\xi_{a}^{(h)}\right) \beta_{a}\left(\xi_{a}^{(h)}\right) \vartheta_{b-1}\left(\bar{\xi}_{a}^{(h)}\right) .
$$

Then the matrix elements of the left and right $\overline{\mathcal{T}}^{(6 \mathrm{VD})}$-eigenstates corresponding to generic eigenvalues:

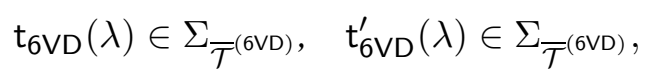

have the following simple form:

$$
\left\langle\mathrm{t}_{6 \mathrm{VD}} \mid \mathrm{t}_{6 \mathrm{VD}}^{\prime}\right\rangle=\delta_{\mathrm{t}, \mathrm{t}^{\prime}} \operatorname{det}_{\mathrm{N}}\left\|\mathcal{F}_{a, b}^{(\mathrm{t}, \mathrm{t})}\right\|, \quad \mathcal{F}_{a, b}^{(\mathrm{t}, \mathrm{t})} \equiv \sum_{l=0}^{1} \bar{Q}_{\mathrm{t}}\left(\xi_{a}^{(l)}\right) Q_{\mathrm{t}}\left(\xi_{a}^{(l)}\right) \vartheta_{b-1}\left(\bar{\xi}_{a}^{(l)}\right),
$$

where we have used the notation $\delta_{\mathrm{t}, \mathrm{t}^{\prime}}=\left\{0\right.$ for $\mathrm{t}_{6 \mathrm{VD}}(\lambda) \neq \mathrm{t}_{6 \mathrm{VD}}^{\prime}(\lambda) \in \Sigma_{\overline{\mathcal{T}}^{(6 \mathrm{VD})}}, 1$ for $\left.\mathrm{t}_{6 \mathrm{VD}}(\lambda)=\mathrm{t}_{6 \mathrm{VD}}^{\prime}(\lambda) \in \Sigma_{\overline{\mathcal{T}}^{(6 \mathrm{VD})}}\right\}$.

Proof. From the SOV-decomposition, we have:

$$
\langle\alpha \mid \beta\rangle=\sum_{h_{1}, \ldots, h_{\mathrm{N}}=0}^{1} \operatorname{det}_{\mathrm{N}} \Theta_{i j}^{(\mathbf{h})} \prod_{a=1}^{\mathrm{N}} \alpha_{a}\left(\xi_{a}^{\left(h_{a}\right)}\right) \beta_{a}\left(\xi_{a}^{\left(h_{a}\right)}\right),
$$

from this formula by using the multilinearity of the determinant w.r.t. the rows we prove the first identity in the proposition. The presence of the delta in (2.122) simply follows from the identities:

$$
\mathrm{t}_{6 \mathrm{VD}}(\lambda)\left\langle\mathrm{t}_{6 \mathrm{VD}} \mid \mathrm{t}_{6 \mathrm{VD}}^{\prime}\right\rangle=\left\langle\mathrm{t}_{6 \mathrm{VD}}\left|\overline{\mathcal{T}}^{(6 \mathrm{VD})}(\lambda \mid \tau)\right| \mathrm{t}_{6 \mathrm{VD}}^{\prime}\right\rangle=\mathrm{t}_{6 \mathrm{VD}}^{\prime}(\lambda)\left\langle\mathrm{t}_{6 \mathrm{VD}} \mid \mathrm{t}_{6 \mathrm{VD}}^{\prime}\right\rangle
$$

where the 1.h.s. is obtained acting on the left state with $\overline{\mathcal{T}}^{(6 \mathrm{VD})}(\lambda \mid \tau)$ and the r.h.s. is obtained acting on the right state with $\overline{\mathcal{T}}^{(6 \mathrm{VD})}(\lambda \mid \tau)$. Indeed for $\mathrm{t}_{6 \mathrm{VD}}(\lambda) \neq \mathrm{t}_{6 \mathrm{VD}}^{\prime}(\lambda)$ the identity implies:

$$
\left\langle\mathrm{t}_{6 \mathrm{VD}} \mid \mathrm{t}_{6 \mathrm{VD}}^{\prime}\right\rangle=0
$$

Finally, for $t_{6 \mathrm{VD}}(\lambda)=\mathrm{t}_{6 \mathrm{VD}}^{\prime}(\lambda)$ the form of the matrix elements just follow recalling that the eigenstates of the transfer matrix are indeed separate states. 


\subsection{Decomposition of the identity in left and right separate basis}

The results of the previous section allow us to write the decomposition of the identity in left and right basis of separate states. In order to define such basis let us introduce the following natural isomorphism between the sets $\{0,1\}^{\mathrm{N}}$ and $\left\{1, \ldots, 2^{\mathrm{N}}\right\}$ :

$$
\varkappa: \mathbf{h} \equiv\left\{h_{1}, \ldots, h_{\mathrm{N}}\right\} \in\{0,1\}^{\mathrm{N}} \rightarrow \varkappa(\mathbf{h}) \equiv 1+\sum_{a=1}^{\mathrm{N}} 2^{(a-1)} h_{a} \in\left\{1, \ldots, 2^{\mathrm{N}}\right\},
$$

and let us denote with $\varkappa_{a}^{-1}(i) \in\{0,1\}$ the entry $a \in\{1, \ldots, N\}$ in the N-tuple $\varkappa^{-1}(i)$ associated by $\varkappa^{-1}$ to any integer $i \in\left\{1, \ldots, 2^{\mathrm{N}}\right\}$. Then, under the conditions:

$$
\begin{aligned}
& \operatorname{det}_{2^{\mathrm{N}}}\left\|M_{i, j}^{(\alpha)}\right\| \neq 0, \quad M_{i, j}^{(\alpha)} \equiv \prod_{a=1}^{\mathrm{N}} \alpha_{a}^{(j)}\left(\xi_{a}^{\left(\varkappa_{a}^{-1}(i)\right)}\right) \forall i, j \in\left\{1, \ldots, 2^{\mathrm{N}}\right\}, \\
& \operatorname{det}_{2^{\mathrm{N}}}\left\|M_{i, j}^{(\beta)}\right\| \neq 0, \quad M_{i, j}^{(\beta)} \equiv \prod_{a=1}^{\mathrm{N}} \beta_{a}^{(j)}\left(\xi_{a}^{\left(\varkappa_{a}^{-1}(i)\right)}\right) \forall i, j \in\left\{1, \ldots, 2^{\mathrm{N}}\right\},
\end{aligned}
$$

the sets of covectors $\left\langle\alpha_{j}\right|$ and vector $\left|\beta_{j}\right\rangle$ defined by:

$$
\begin{aligned}
& \left\langle\alpha_{j}\right|=\sum_{h_{1}, \ldots, h_{\mathrm{N}}=0}^{1} \prod_{a=1}^{\mathrm{N}} \alpha_{a}^{(j)}\left(\xi_{a}^{\left(h_{a}\right)}\right) \operatorname{det}_{\mathrm{N}} \Theta_{i j}^{(\mathbf{h})}\left\langle h_{1}, \ldots, h_{\mathrm{N}}\right| \quad \forall j \in\left\{1, \ldots, 2^{\mathrm{N}}\right\}, \\
& \left|\beta_{j}\right\rangle=\sum_{h_{1}, \ldots, h_{\mathrm{N}}=0}^{1} \prod_{a=1}^{\mathrm{N}} \beta_{a}^{(j)}\left(\xi_{a}^{\left(h_{a}\right)}\right) \operatorname{det}_{\mathrm{N}} \Theta_{i j}^{(\mathbf{h})}\left|h_{1}, \ldots, h_{\mathrm{N}}\right\rangle \quad \forall j \in\left\{1, \ldots, 2^{\mathrm{N}}\right\},
\end{aligned}
$$

generate separate basis of $\overline{\mathbb{D}}_{(6 \mathrm{VD}), \mathrm{N}}^{\mathcal{L}}$ and $\overline{\mathbb{D}}_{(6 \mathrm{VD}), \mathrm{N}}^{\mathcal{R}}$, respectively. Moreover, defined:

$$
\mathbf{N}_{\mathbf{h}} \equiv\left\{\mathbf{k} \in\{0,1\}^{\mathrm{N}}: \operatorname{det}_{\mathrm{N}}\left\|\mathcal{F}_{a, b}^{\left(\alpha_{\varkappa(\mathbf{h})}, \beta_{\varkappa(\mathbf{k})}\right)}\right\| \neq 0\right\},
$$

then the following decomposition of the identity is implied on these separate basis:

$$
\mathbb{I}=\sum_{\mathbf{h} \in\{0,1\}^{N}} \sum_{\mathbf{k} \in \mathbf{N}_{\mathbf{h}}}\left(\operatorname{det}_{N}\left\|\mathcal{F}_{a, b}^{\left(\alpha_{\varkappa(\mathbf{h})}, \beta_{\varkappa(\mathbf{k})}\right)}\right\|\right)^{-1}\left|\beta_{\varkappa(\mathbf{k})}\right\rangle\left\langle\alpha_{\varkappa(\mathbf{h})}\right|,
$$

which reads:

$$
\mathbb{I}=\sum_{\mathrm{t}(\lambda) \in \Sigma_{\overline{\mathcal{T}}^{(6 \mathrm{VD})}}}\left(\operatorname{det}_{N}\left\|\mathcal{F}_{a, b}^{(\mathrm{t}, \mathrm{t})}\right\|\right)^{-1}\left|\mathrm{t}_{6 \mathrm{VD}}\right\rangle\left\langle\mathrm{t}_{6 \mathrm{VD}}\right|,
$$

for the representations for which the antiperiodic transfer matrix $\overline{\mathcal{T}}^{(6 \mathrm{VD})}(\lambda \mid \tau)$ is proven to be diagonalizable.

\section{On the periodic 8-vertex spectrum and connection with SOV}

In this section we will analyze the connection between the spectral problem of the periodic 8-vertex transfer matrix on chains with an odd number of quantum sites and the one of the antiperiodic dynamical 6-vertex transfer matrix. The Baxter's gauge transformations are used together with the functional characterization of the 8-vertex transfer matrix to get central information on the spectrum (eigenvalues and eigenstates) of this model by our SOV results. 


\subsection{The 8-vertex model}

Let us recall the characterization in terms of QISM of the XYZ spin-1/2 quantum chain. The 8-vertex R-matrix reads:

$$
R_{0 a}^{(8 \mathrm{~V})}(\lambda)=\left(\begin{array}{cccc}
\mathrm{a}(\lambda) & 0 & 0 & \mathrm{~d}(\lambda) \\
0 & \mathrm{~b}(\lambda) & \mathrm{c}(\lambda) & 0 \\
0 & \mathrm{c}(\lambda) & \mathrm{b}(\lambda) & 0 \\
\mathrm{~d}(\lambda) & 0 & 0 & \mathrm{a}(\lambda)
\end{array}\right)
$$

where:

$$
\begin{array}{ll}
\mathrm{a}(\lambda)=\frac{2 \theta_{4}(\eta \mid 2 \omega) \theta_{1}(\lambda+\eta \mid 2 \omega) \theta_{4}(\lambda \mid 2 \omega)}{\theta_{2}(0 \mid \omega) \theta_{4}(0 \mid 2 \omega)}, & \mathrm{b}(\lambda)=\frac{2 \theta_{4}(\eta \mid 2 \omega) \theta_{1}(\lambda \mid 2 \omega) \theta_{4}(\lambda+\eta \mid 2 \omega)}{\theta_{2}(0 \mid \omega) \theta_{4}(0 \mid 2 \omega)}, \\
\mathrm{c}(\lambda)=\frac{2 \theta_{1}(\eta \mid 2 \omega) \theta_{4}(\lambda \mid 2 \omega) \theta_{4}(\lambda+\eta \mid 2 \omega)}{\theta_{2}(0 \mid \omega) \theta_{4}(0 \mid 2 \omega)}, & \mathrm{d}(\lambda)=\frac{2 \theta_{1}(\eta \mid 2 \omega) \theta_{1}(\lambda+\eta \mid 2 \omega) \theta_{1}(\lambda \mid 2 \omega)}{\theta_{2}(0 \mid \omega) \theta_{4}(0 \mid 2 \omega)},
\end{array}
$$

is solution of the Yang-Baxter equation:

$$
R_{12}^{(8 \mathrm{~V})}\left(\lambda_{12}\right) R_{1 a}^{(8 \mathrm{~V})}\left(\lambda_{1}\right) R_{2 a}^{(8 \mathrm{~V})}\left(\lambda_{2}\right)=R_{2 a}^{(8 \mathrm{~V})}\left(\lambda_{2}\right) R_{1 a}^{(8 \mathrm{~V})}\left(\lambda_{1}\right) R_{12}^{(8 \mathrm{~V})}\left(\lambda_{12}\right)
$$

Then the monodromy matrix of the spin- $1 / 2$ representations is defined by:

$$
\mathrm{M}_{0}^{(8 \mathrm{~V})}(\lambda) \equiv R_{0 \mathrm{~N}}^{(8 \mathrm{~V})}\left(\lambda-\xi_{\mathrm{N}}\right) \cdots R_{01}^{(8 \mathrm{~V})}\left(\lambda-\xi_{1}\right) \equiv\left(\begin{array}{cc}
\mathrm{A}^{(8 \mathrm{~V})}(\lambda) & \mathrm{B}^{(8 \mathrm{~V})}(\lambda) \\
\mathrm{C}^{(8 \mathrm{~V})}(\lambda) & \mathrm{D}^{(8 \mathrm{~V})}(\lambda)
\end{array}\right)
$$

with the parameters $\xi_{a}$ which are the inhomogeneities. The monodromy matrix $\mathrm{M}_{0}^{(8 \mathrm{~V})}(\lambda)$ is itself solution of the Yang-Baxter equation:

$$
R_{12}^{(8 \mathrm{~V})}\left(\lambda_{12}\right) \mathrm{M}_{1}^{(8 \mathrm{~V})}\left(\lambda_{1}\right) \mathrm{M}_{2}^{(8 \mathrm{~V})}\left(\lambda_{2}\right)=\mathrm{M}_{2}^{(8 \mathrm{~V})}\left(\lambda_{2}\right) \mathrm{M}_{1}^{(8 \mathrm{~V})}\left(\lambda_{1}\right) R_{12}^{(8 \mathrm{~V})}\left(\lambda_{12}\right),
$$

and then the corresponding transfer matrix:

$$
\mathrm{T}^{(8 \mathrm{~V})}(\lambda)=\operatorname{tr}_{0} \mathrm{M}_{0}^{(8 \mathrm{~V})}(\lambda)
$$

defines a one parameter family of commuting operators; the Hamiltonian of the XYZ spin-1/2 quantum chain is obtained in the homogeneous limit by:

$$
H_{X Y Z}=\left.2 \sinh \eta \frac{\partial \ln \mathrm{T}^{(8 \mathrm{~V})}(\lambda)}{\partial \lambda}\right|_{\lambda=0, \xi_{n}=0}-\mathrm{N} \cosh \eta .
$$

\subsection{Elementary properties of the periodic 8-vertex transfer matrix}

Let us describe some elementary properties of the periodic 8-vertex transfer matrix which allow a first characterization of the spectrum evidencing its connection to the antiperiodic dynamical 6-vertex spectrum in the case of odd chains.

Lemma 3.1. In the 8-vertex Yang-Baxter algebra, we can introduce the following central quantum determinant:

$$
\begin{aligned}
\operatorname{det}_{q} \mathrm{M}^{(8 \mathrm{~V})}(\lambda) & \equiv\left(\mathrm{A}^{(8 \mathrm{~V})}(\lambda) \mathrm{D}^{(8 \mathrm{~V})}(\lambda-\eta)-\mathrm{B}^{(8 \mathrm{~V})}(\lambda) \mathrm{C}^{(8 \mathrm{~V})}(\lambda-\eta)\right) \\
& =\left(\mathrm{D}^{(8 \mathrm{~V})}(\lambda) \mathrm{A}^{(8 \mathrm{~V})}(\lambda-\eta)-\mathrm{C}^{(8 \mathrm{~V})}(\lambda) \mathrm{B}^{(8 \mathrm{~V})}(\lambda-\eta)\right) \\
& =\mathrm{A}(\lambda) \mathrm{D}(\lambda-\eta),
\end{aligned}
$$


and the following inversion formula holds:

$$
\left[\mathrm{M}_{0}^{(8 \mathrm{~V})}(\lambda)\right]^{-1}=\frac{\sigma_{0}^{y}\left[\mathrm{M}_{0}^{(8 \mathrm{~V})}(\lambda-\eta)\right]^{t_{0}} \sigma_{0}^{y}}{\mathrm{~A}(\lambda) \mathrm{D}(\lambda-\eta)}
$$

Proof. The centrality of the quantum determinant is a well known property in the 6-vertex Yang-Baxter algebra and it is possible to extend it to the 8-vertex case. The proof is given by proving the statement for the generic quantum site $n$ and then showing that the product of the local quantum determinants reproduce the complete one. Let us introduce the notation:

$$
\begin{aligned}
\operatorname{det}_{q} R_{0 n}^{(8 \mathrm{~V})}(\lambda) & =\left(R_{0 n}^{(8 \mathrm{~V})}\right)_{11}(\lambda)\left(R_{0 n}^{(6 \mathrm{VD})}\right)_{22}(\lambda-\eta) \\
& -\left(R_{0 n}^{(6 \mathrm{VD})}\right)_{12}(\lambda)\left(R_{0 n}^{(6 \mathrm{VD})}\right)_{21}(\lambda-\eta),
\end{aligned}
$$

its explicit form reads:

$$
\operatorname{det}_{q} R_{0, n}^{(8 \mathrm{~V})}\left(\lambda-\xi_{n}\right)=\left(\begin{array}{cc}
\mathrm{a}(\lambda) \mathrm{b}(\lambda-\eta)-\mathrm{d}(\lambda) \mathrm{d}(\lambda-\eta) & 0 \\
0 & \mathrm{~b}(\lambda) \mathrm{a}(\lambda-\eta)-\mathrm{c}(\lambda) \mathrm{c}(\lambda-\eta)
\end{array}\right),
$$

then all we need to prove are the following identities:

$$
\mathrm{a}(\lambda) \mathrm{b}(\lambda-\eta)-\mathrm{d}(\lambda) \mathrm{d}(\lambda-\eta)=\mathrm{b}(\lambda) \mathrm{a}(\lambda-\eta)-\mathrm{c}(\lambda) \mathrm{c}(\lambda-\eta)=a\left(\lambda-\xi_{n}\right) a\left(\lambda-\xi_{n}-2 \eta\right)
$$

which trivially follow once we use the formulae 25 :

$$
\begin{aligned}
& \theta_{1}(x+y \mid 2 \omega) \theta_{1}(x-y \mid 2 \omega) \theta_{4}^{2}(0)=\theta_{3}^{2}(x \mid 2 \omega) \theta_{2}^{2}(y \mid 2 \omega)-\theta_{2}^{2}(x \mid 2 \omega) \theta_{3}^{2}(y \mid 2 \omega), \\
& \theta_{4}(x+y \mid 2 \omega) \theta_{4}(x-y \mid 2 \omega) \theta_{4}^{2}(0)=\theta_{4}^{2}(x \mid 2 \omega) \theta_{4}^{2}(y \mid 2 \omega)-\theta_{1}^{2}(x \mid 2 \omega) \theta_{1}^{2}(y \mid 2 \omega), \\
& \theta_{1}(x \mid \omega) \theta_{2}(x \mid \omega)=\theta_{1}(x+y \mid 2 \omega) \theta_{4}(x-y \mid 2 \omega)+\theta_{4}(x+y \mid 2 \omega) \theta_{1}(x-y \mid 2 \omega) .
\end{aligned}
$$

Finally, the inversion formula (3.12) follows from the quantum determinant formulae and from the identities:

$$
\mathrm{A}^{(8 \mathrm{~V})}(\lambda) \mathrm{B}^{(8 \mathrm{~V})}(\lambda-\eta)-\mathrm{B}^{(8 \mathrm{~V})}(\lambda) \mathrm{A}^{(8 \mathrm{~V})}(\lambda-\eta)=0, \quad \mathrm{D}^{(8 \mathrm{~V})}(\lambda) \mathrm{C}^{(8 \mathrm{~V})}(\lambda-\eta)-\mathrm{C}^{(8 \mathrm{~V})}(\lambda) \mathrm{D}^{(8 \mathrm{~V})}(\lambda-\eta)=0,
$$

which directly follows from the 8-vertex Yang-Baxter equations (3.6).

Moreover, it holds:

Lemma 3.2. The following products $\left(\mathrm{M}^{(8 \mathrm{~V})}\left(\xi_{n}^{(0)}\right)\right)_{h, j}\left(\mathrm{M}^{(8 \mathrm{~V})}\left(\xi_{n}^{(1)}\right)\right)_{k, j}$ and $\left(\mathrm{M}^{(8 \mathrm{~V})}\left(\xi_{n}^{(1)}\right)\right)_{j, h}\left(\mathrm{M}^{(8 \mathrm{~V})}\left(\xi_{n}^{(0)}\right)\right)_{j, k}$ of the elements of the 8-vertex monodromy matrix vanish for any $n \in\{1, \ldots, \mathrm{N}\}$ if $h=k$ and the following identities hold:

$$
\begin{aligned}
& \mathrm{A}^{(8 \mathrm{~V})}\left(\xi_{n}^{(0)}\right) \mathrm{D}^{(8 \mathrm{~V})}\left(\xi_{n}^{(1)}\right)=-\mathrm{C}^{(8 \mathrm{~V})}\left(\xi_{n}^{(0)}\right) \mathrm{B}^{(8 \mathrm{~V})}\left(\xi_{n}^{(1)}\right), \\
& \mathrm{D}^{(8 \mathrm{~V})}\left(\xi_{n}^{(0)}\right) \mathrm{A}^{(8 \mathrm{~V})}\left(\xi_{n}^{(1)}\right)=-\mathrm{B}^{(8 \mathrm{~V})}\left(\xi_{n}^{(0)}\right) \mathrm{C}^{(8 \mathrm{~V})}\left(\xi_{n}^{(1)}\right) .
\end{aligned}
$$

Proof. This lemma is a trivial generalization to the 8-vertex Yang-Baxter algebra of the results known in the 6vertex case, see for example [66]. Both in the 8-vertex and 6-vertex case these results are simple consequences of the reconstruction formulae of local operators in terms of matrix elements of the monodromy matrix first proven for

\footnotetext{
${ }^{25}$ Respectively, equations 7,10 and 2 at page 881 of [158].
} 
the 6-vertex case in [46] and then extended also to the 8-vertex case in [53]. In the 8-vertex case the reconstructions read:

$$
\begin{aligned}
X_{n} & =\prod_{b=1}^{n-1} \mathrm{~T}^{(8 \mathrm{~V})}\left(\xi_{b}^{(0)}\right) \operatorname{tr}_{0}\left(\mathrm{M}^{(8 \mathrm{~V})}\left(\xi_{n}^{(0)}\right) X_{0}\right) \prod_{b=1}^{n} \frac{\mathrm{T}^{(8 \mathrm{~V})}\left(\xi_{b}^{(1)}\right)}{\operatorname{det}_{q} \mathrm{M}^{(8 \mathrm{~V})}\left(\xi_{b}^{(0)}\right)} \\
& =\prod_{b=1}^{n} \mathrm{~T}^{(8 \mathrm{~V})}\left(\xi_{b}^{(0)}\right) \frac{\operatorname{tr}_{0}\left(\mathrm{M}^{(8 \mathrm{~V})}\left(\xi_{n}^{(1)}\right) \sigma_{0}^{(y)} X_{0}^{t_{0}} \sigma_{0}^{(y)}\right)}{\operatorname{det}_{q} \mathrm{M}^{(8 \mathrm{~V})}\left(\xi_{n}^{(0)}\right)} \prod_{b=1}^{n-1} \frac{\mathrm{T}^{(8 \mathrm{~V})}\left(\xi_{b}^{(1)}\right)}{\operatorname{det}_{q} \mathrm{M}^{(8 \mathrm{~V})}\left(\xi_{b}^{(0)}\right)}
\end{aligned}
$$

where $X_{n}$ is a local operator on the quantum space $n$, i.e. it acts as the identity on any quantum space associate to a site $m \neq n$ and as the $2 \times 2$ matrix $X$ on the quantum space in the site $n$, while $X_{0}$ is the $2 \times 2$ matrix $X$ on the auxiliary space. Let us consider for example the following identity:

$$
\left(\begin{array}{ll}
0 & 0 \\
0 & 1
\end{array}\right)_{n}=X_{n} X_{n}=Y_{n} Z_{n}
$$

where:

$$
X_{n}=\left(\begin{array}{ll}
0 & 0 \\
0 & 1
\end{array}\right)_{n}, Y_{n}=\left(\begin{array}{ll}
0 & 0 \\
1 & 0
\end{array}\right)_{n} \text { and } Z_{n}=\left(\begin{array}{cc}
0 & 1 \\
0 & 0
\end{array}\right)_{n},
$$

then the identity (3.21) simply follows by using for the first $X_{n}$ and the $Y_{n}$ the reconstruction (3.22) while for the second $X_{n}$ and the $Z_{n}$ the reconstruction (3.23). All the other identities in this lemma are proven similarly by taking the product of a couple of local operators and using for them the two reconstructions.

\subsection{On the periodic 8-vertex transfer matrix eigenvalues}

The previous two lemmas allow to prove some preliminary characterization of the periodic 8-vertex eigenvalues as presented in the following proposition:

Proposition 3.1. The periodic 8-vertex transfer matrix of a chain with $\mathrm{N}$ quantum sites satisfies the following properties:

$$
\mathrm{T}^{(8 \mathrm{~V})}\left(\xi_{n}^{(0)}\right) \mathrm{T}^{(8 \mathrm{~V})}\left(\xi_{n}^{(1)}\right)=\operatorname{det}{ }_{q} \mathrm{M}^{(8 \mathrm{~V})}\left(\xi_{n}^{(0)}\right) \quad \forall n \in\{1, \ldots, \mathrm{N}\}
$$

and

$$
\mathrm{T}^{(8 \mathrm{~V})}(\lambda+\pi)=(-1)^{\mathrm{N}} \mathbf{T}^{(8 \mathrm{~V})}(\lambda), \mathbf{T}^{(8 \mathrm{~V})}(\lambda+\pi \omega)=\left(-e^{-i(2 \lambda+\pi w)}\right)^{\mathrm{N}} e^{-2 i\left(t_{0}-\sum_{a=1}^{\mathrm{N}} \xi_{a}\right)} \mathbf{T}^{(8 \mathrm{~V})}(\lambda) .
$$

Then the 8-vertex eigenvalues are elliptic polynomials (or theta functions) of degree $\mathrm{N}$ and character $e^{-2 i\left(t_{\boldsymbol{o}}-\sum_{a=1}^{N} \xi_{a}\right)}$ and they admit the following interpolation formula:

$$
\mathrm{t}_{8 \mathrm{~V}}(\lambda)=\sum_{a=1}^{\mathrm{N}} \frac{\theta\left(t_{\boldsymbol{0}}-\lambda+\xi_{a}\right)}{\theta\left(t_{\boldsymbol{0}}\right)} \prod_{b \neq a} \frac{\theta\left(\lambda-\xi_{b}\right)}{\theta\left(\xi_{a}-\xi_{b}\right)} \mathrm{t}_{8 \mathrm{~V}}\left(\xi_{a}\right),
$$

where the $\operatorname{tav}_{8 \mathrm{~V}}\left(\xi_{a}\right)$ are solutions of the discrete system of equations:

$$
\mathrm{t}_{8 \mathrm{~V}}\left(\xi_{a}^{(0)}\right) \mathrm{t}_{8 \mathrm{~V}}\left(\xi_{a}^{(1)}\right)=\mathrm{A}\left(\xi_{a}^{(0)}\right) \mathrm{D}\left(\xi_{a}^{(1)}\right), \quad \forall a \in\{1, \ldots, \mathrm{N}\} .
$$

Proof. The Lemma 3.2 in particular implies the annihilation identities:

$$
\mathrm{A}^{(8 \mathrm{~V})}\left(\xi_{n}^{(0)}\right) \mathrm{A}^{(8 \mathrm{~V})}\left(\xi_{n}^{(1)}\right)=\mathrm{D}^{(8 \mathrm{~V})}\left(\xi_{n}^{(0)}\right) \mathrm{D}^{(8 \mathrm{~V})}\left(\xi_{n}^{(1)}\right)=0,
$$


from which we can write:

$$
\mathbf{T}^{(8 \mathrm{~V})}\left(\xi_{n}^{(0)}\right) \mathrm{T}^{(8 \mathrm{~V})}\left(\xi_{n}^{(1)}\right)=\mathrm{A}^{(8 \mathrm{~V})}\left(\xi_{n}^{(0)}\right) \mathrm{D}^{(8 \mathrm{~V})}\left(\xi_{n}^{(1)}\right)+\mathrm{D}^{(8 \mathrm{~V})}\left(\xi_{n}^{(0)}\right) \mathrm{A}^{(8 \mathrm{~V})}\left(\xi_{n}^{(1)}\right)
$$

and then eliminating in the above equation $\mathrm{D}^{(8 \mathrm{~V})}\left(\xi_{n}^{(0)}\right) \mathrm{A}^{(8 \mathrm{~V})}\left(\xi_{n}^{(1)}\right)$ by using $(\underline{3.201})$ or $\mathrm{A}^{(8 \mathrm{~V})}\left(\xi_{n}^{(0)}\right) \mathrm{D}^{(8 \mathrm{~V})}\left(\xi_{n}^{(1)}\right)$ by using (3.21) we get the identity (3.26).

Let us observe now that by using the identities 26 :

$$
\begin{array}{ll}
\theta_{1}(x+\pi \mid 2 \omega)=-\theta_{1}(x \mid 2 \omega), & \theta_{1}(x+\pi \omega \mid 2 \omega)=i e^{-i(\lambda+\pi w / 2)} \theta_{4}(x \mid 2 \omega) \\
\theta_{4}(x+\pi \mid 2 \omega)=\theta_{4}(x \mid 2 \omega), & \theta_{4}(x+\pi \omega \mid 2 \omega)=i e^{-i(\lambda+\pi w / 2)} \theta_{1}(x \mid 2 \omega)
\end{array}
$$

it is simple to show that the coefficients of the 8 -vertex $R$-matrix satisfy the following transformation properties:

$$
\begin{aligned}
& \mathrm{a}(\lambda+\pi \omega)=-e^{-i(2 \lambda+\pi w)} e^{-i \eta} \mathrm{b}(\lambda), \quad \mathrm{a}(\lambda+\pi)=-\mathrm{a}(\lambda), \mathrm{d}(\lambda+\pi)=\mathrm{d}(\lambda), \\
& \mathrm{c}(\lambda+\pi \omega)=-e^{-i(2 \lambda+\pi w)} e^{-i \eta} \mathrm{d}(\lambda), \quad \mathrm{b}(\lambda+\pi)=-\mathrm{b}(\lambda), \mathrm{c}(\lambda+\pi)=\mathrm{c}(\lambda),
\end{aligned}
$$

which are equivalent to the following identities on the 8 -vertex $R$-matrix:

$$
R_{0 a}^{(8 \mathrm{~V})}(\lambda+\pi \omega)=-e^{-i(2 \lambda+\pi w)} e^{-i \eta} \sigma_{0}^{(x)} R_{0 a}^{(8 \mathrm{~V})}(\lambda) \sigma_{0}^{(x)}, \quad R_{0 a}^{(8 \mathrm{~V})}(\lambda+\pi)=-\sigma_{0}^{(z)} R_{0 a}^{(8 \mathrm{~V})}(\lambda) \sigma_{0}^{(z)} .
$$

Then, the monodromy matrix satisfy the identities:

$$
\begin{aligned}
\mathrm{M}_{0}^{(8 \mathrm{~V})}(\lambda+\pi w) & =\left(-e^{-i(2 \lambda+\pi w)}\right)^{\mathrm{N}} e^{-2 i\left(t_{\mathbf{0}}-\sum_{a=1}^{\mathrm{N}} \xi_{a}\right)} \sigma_{0}^{(x)} \mathrm{M}_{0}^{(8 \mathrm{~V})}(\lambda) \sigma_{0}^{(x)}, \\
\mathrm{M}_{0}^{(8 \mathrm{~V})}(\lambda+\pi) & =(-1)^{\mathrm{N}} \sigma_{0}^{(z)} \mathrm{M}_{0}^{(8 \mathrm{~V})}(\lambda) \sigma_{0}^{(z)},
\end{aligned}
$$

from which (3.26) follows by the cyclicity of the trace. The formula (3.28) is the interpolation formula [159] for elliptic polynomials of degree $\mathrm{N}$ and character $e^{-2 i\left(t_{\mathbf{0}}-\sum_{a=1}^{\mathrm{N}} \xi_{a}\right)}$ while (3.29) is the rewiting of (3.26) for the periodic 8-vertex eigenvalues.

Remark 1. Let us notice that the above results hold for both the even and the odd quantum chains. In the odd case these results implies that the set of the periodic 8-vertex transfer matrix eigenvalues is contained in the set of the antiperiodic dynamical 6-vertex transfer matrix eigenvalues. As we have proven that the antiperiodic dynamical 6-vertex transfer matrix has simple spectrum for general values of the inhomogeneities, then differences in these sets of eigenvalues can be only produced from a degeneracy of the periodic 8-vertex transfer matrix eigenvalues. A preliminary analysis based on direct diagonalization shows that the periodic 8-vertex transfer matrix spectrum is double degenerate for $\mathrm{N}=1,3$. These cases are considered in the appendix where it is also given a direct verification of the statement proven in Theorem 2.3 that the system of equations defined by (2.103) and (2.104) characterize the complete set of the antiperiodic dynamical 6-vertex transfer matrix eigenvalues.

\subsection{Gauge transformation from 8-vertex to dynamical 6-vertex models}

In the case of an even chain the spectral problem of the 8-vertex transfer matrix $\mathrm{T}^{(8 \mathrm{~V})}(\lambda)$ has been reduced to the one of the periodic dynamical 6-vertex transfer matrix by the gauge transformations introduced by Baxter in [22]. In detail the following gauge transformation exists:

$$
R_{0 a}^{(8 \mathrm{~V})}\left(\lambda_{12}\right) S_{0}\left(\lambda_{1} \mid \tau\right) S_{a}\left(\lambda_{2} \mid \tau+\eta \sigma_{0}^{z}\right)=S_{a}\left(\lambda_{2} \mid \tau\right) S_{0}\left(\lambda_{1} \mid \tau+\eta \sigma_{a}^{z}\right) R_{0 a}^{(6 \mathrm{VD})}\left(\lambda_{12} \mid \tau\right),
$$

\footnotetext{
${ }^{26}$ See the equations $8.182-1,8.182-3$ and $8.183-5,8.183-6$ at page 878 of [158].
} 
which for the monodromy matrices reads:

$$
\mathrm{M}_{0}^{(8 \mathrm{~V})}(\lambda) S_{0}(\lambda \mid \tau) S_{q}\left(\tau+\eta \sigma_{0}^{z}\right)=S_{q}(\tau) S_{0}(\lambda \mid \tau+\eta \mathrm{S}) \mathrm{M}_{0}^{(6 \mathrm{VD})}(\lambda \mid \tau)
$$

where:

$$
S_{0}(\lambda \mid \tau) \equiv\left(\begin{array}{cc}
\theta_{2}(-\lambda+\tau \mid 2 w) & \theta_{2}(\lambda+\tau \mid 2 w) \\
\theta_{3}(-\lambda+\tau \mid 2 w) & \theta_{3}(\lambda+\tau \mid 2 w)
\end{array}\right)_{0}
$$

and:

$$
S_{q}(\tau) \equiv S_{1}\left(\xi_{1} \mid \tau\right) \cdots S_{\mathrm{N}}\left(\xi_{\mathrm{N}} \mid \tau+\eta \sum_{a=1}^{\mathrm{N}-1} \sigma_{a}^{z}\right)
$$

Then we can prove:

Lemma 3.3. In a chain with an odd number of quantum sites the periodic 8-vertex transfer matrix has the following right action on the states of $\overline{\mathbb{D}}_{(6 \mathrm{VD}), \mathrm{N}}^{\mathcal{R}}$ :

$$
\mathrm{T}^{(8 \mathrm{~V})}(\lambda) S_{q}(\tau)=S_{q}(\tau-\eta) \mathrm{C}(\lambda \mid \tau-\eta)+S_{q}(\tau+\eta) \mathrm{B}(\lambda \mid \tau+\eta) .
$$

Proof. To prove (3.43) let us first rewrite the gauge transformation (3.40) as it follows:

$$
\begin{aligned}
S_{0}(\lambda+\eta \mid \tau) S_{q}(\tau & \left.+\eta \sigma_{0}^{z}\right)\left(\begin{array}{cc}
\mathrm{D}(\lambda \mid \tau+\eta) & -\mathrm{B}(\lambda \mid \tau+\eta) \\
-\mathrm{C}(\lambda \mid \tau-\eta) & \mathrm{A}(\lambda \mid \tau-\eta)
\end{array}\right) \frac{\theta(\tau+\eta \mathrm{S})}{\theta(\tau)}= \\
& =\left(\begin{array}{cc}
\mathrm{D}^{(8 \mathrm{~V})}(\lambda) & -\mathrm{B}^{(8 \mathrm{~V})}(\lambda) \\
-\mathrm{C}^{(8 \mathrm{~V})}(\lambda) & \mathrm{A}^{(8 \mathrm{~V})}(\lambda)
\end{array}\right) S_{q}(\tau) S_{0}(\lambda+\eta \mid \tau+\eta \mathrm{S}),
\end{aligned}
$$

obtained by multiply both sides of (3.40) from the right by the inverse of $\mathrm{M}_{0}^{(6 \mathrm{VD})}\left(\lambda_{1} \mid \tau\right)$, as defined in (2.29), and from the left by the inverse of $\mathrm{M}_{0}^{(8 \mathrm{~V})}(\lambda)$, as defined in (3.12), and finally doing the change of variable $\lambda \rightarrow \lambda+\eta$. The gauge transformation (3.44) can be further rewritten as it follows:

$$
\begin{array}{r}
S_{0}(\lambda+\eta \mid-\tau) S_{q}\left(\tau-\eta \sigma_{0}^{z}\right)\left(\begin{array}{cc}
-\mathrm{C}(\lambda \mid \tau-\eta) & \mathrm{A}(\lambda \mid \tau-\eta) \\
\mathrm{D}(\lambda \mid \tau+\eta) & -\mathrm{B}(\lambda \mid \tau+\eta)
\end{array}\right) \frac{\theta(\tau+\eta \mathrm{S})}{\theta(\tau)}= \\
=\left(\begin{array}{cc}
\mathrm{D}^{(8 \mathrm{~V})}(\lambda) & -\mathrm{B}^{(8 \mathrm{~V})}(\lambda) \\
-\mathrm{C}^{(8 \mathrm{~V})}(\lambda) & \mathrm{A}^{(8 \mathrm{~V})}(\lambda)
\end{array}\right) S_{q}(\tau) S_{0}(\lambda+\eta \mid \tau+\eta \mathrm{S}),
\end{array}
$$

by using the identity:

$$
S_{0}(\lambda \mid-\tau)=S_{0}(-\lambda \mid \tau)=S_{0}(\lambda \mid \tau) \sigma_{0}^{x} .
$$

We can take now the trace w.r.t. the auxiliary space 0 and we get:

$$
\begin{aligned}
\mathrm{T}^{(8 \mathrm{~V})}(\lambda) S_{q}(\tau)= & \operatorname{tr}_{0}\left\{S_{q}\left(\tau-\eta \sigma_{0}^{z}\right)\left(\begin{array}{cc}
-\mathrm{C}(\lambda \mid \tau-\eta) & \mathrm{A}(\lambda \mid \tau-\eta) \\
\mathrm{D}(\lambda \mid \tau+\eta) & -\mathrm{B}(\lambda \mid \tau+\eta)
\end{array}\right)\right. \\
& \left.\times \frac{\theta(\tau+\eta \mathrm{S})}{\theta(\tau)}\left[S_{0}(\lambda+\eta \mid \tau+\eta \mathrm{S})\right]^{-1} S_{0}(\lambda+\eta \mid-\tau)\right\},
\end{aligned}
$$

where we have used the commutativity:

$$
\left[\overline{\mathrm{M}}_{0}^{(6 \mathrm{VD})}(\lambda \mid \tau), \tau\right]=0
$$

and the cyclicity of the trace to move $S_{0}(\lambda+\eta \mid-\tau)$. It is central to remark that $S_{0}(\lambda+\eta \mid \tau+\eta \mathrm{S})$ is an invertible matrix in the auxiliary space on any state of $\overline{\mathbb{D}}_{(6 \mathrm{VD}), N}^{\mathcal{R}}$ and so the identity $(3.47)$ is well defined on $\overline{\mathbb{D}}_{(6 \mathrm{VD}), N}^{\mathcal{R}}$ and using it we get our result (3.43) being the eigenvalues of $\tau$ and $-\tau-\eta S$ coinciding on any left state of $\overline{\mathbb{D}}_{(6 \mathrm{VD}), N}^{\mathcal{R}}$. 


\subsection{On the periodic 8-vertex transfer matrix spectrum by SOV}

\subsubsection{Connections between periodic 8-vertex and antiperiodic dynamical 6-vertex spectrum}

Let us define $\overline{\mathbb{P}}_{N}^{\mathcal{R}} \equiv \sum_{s=-N}^{N}\langle t(\mathrm{~s})|$, by the definition of scalar product given in Section 2.1.1, the action of $\overline{\mathbb{P}}_{N}^{\mathcal{R}}$ reduces the dynamical-spin vector space $\mathbb{D}_{(6 \mathrm{VD}), N}^{\mathcal{R}}$ to the $2^{\mathrm{N}}$-dimensional spin vector space $\mathbb{S}_{N}^{\mathcal{R}}=\overline{\mathbb{P}}_{N}^{\mathcal{R}} \overline{\mathbb{D}}_{(6 \mathrm{VD}), \mathrm{N}}^{\mathcal{R}}$ and relates the generic vectors of their basis by $\overline{\mathbb{P}}_{\mathrm{N}}^{\mathcal{R}} \otimes_{n=1}^{\mathrm{N}}\left|n, h_{n}\right\rangle \otimes\left|t_{\mathrm{h}}\right\rangle=\otimes_{n=1}^{\mathrm{N}}\left|n, h_{n}\right\rangle$. Moreover, let us introduce the pure spin operator $S_{q}^{\mathcal{R}} \in \operatorname{End}\left(\mathbb{S}_{N}^{\mathcal{R}}\right)$ by the following actions:

$$
\begin{aligned}
\mathrm{S}_{q}^{\mathcal{R}} \otimes_{n=1}^{\mathrm{N}}\left|n, h_{n}\right\rangle \equiv & S_{1}\left(\xi_{1} \mid-\frac{\eta}{2} \sum_{a=1}^{\mathrm{N}} \sigma_{a}^{z}\right) \cdots S_{n}\left(\xi_{n} \mid \frac{\eta}{2} \sum_{a=1}^{n-1} \sigma_{a}^{z}-\frac{\eta}{2} \sum_{a=n}^{\mathrm{N}} \sigma_{a}^{z}\right) \\
& \cdots S_{\mathrm{N}}\left(\xi_{\mathrm{N}} \mid \frac{\eta}{2} \sum_{a=1}^{\mathrm{N}-1} \sigma_{a}^{z}-\frac{\eta}{2} \sigma_{\mathrm{N}}^{z}\right) \otimes_{n=1}^{\mathrm{N}}\left|n, h_{n}\right\rangle,
\end{aligned}
$$

on the spin basis of the pure spin quantum space $\mathbb{S}_{\mathrm{N}}^{\mathcal{R}}$, then we have:

\section{Proposition 3.2.}

I) On any vector of $\overline{\mathbb{D}}_{(6 \mathrm{VD}), \mathrm{N}}^{\mathcal{R}}$ it holds:

$$
\overline{\mathbb{P}}_{\mathrm{N}}^{\mathcal{R}} S_{q}(\tau)=\mathrm{S}_{q}^{\mathcal{R}} \overline{\mathbb{P}}_{\mathrm{N}}^{\mathcal{R}}
$$

II) The following identities hold:

$$
\overline{\mathbb{P}}_{\mathrm{N}}^{\mathcal{R}} S_{q}(\tau-\eta) \mathrm{C}(\lambda \mid \tau-\eta)=\mathrm{S}_{q}^{\mathcal{R}} \overline{\mathbb{P}}_{\mathrm{N}}^{\mathcal{R}} \mathcal{C}(\lambda \mid \tau), \quad \overline{\mathbb{P}}_{\mathrm{N}}^{\mathcal{R}} S_{q}(\tau+\eta) \mathrm{B}(\lambda \mid \tau+\eta)=\mathrm{S}_{q}^{\mathcal{R}} \overline{\mathbb{P}}_{\mathrm{N}}^{\mathcal{R}} \mathcal{B}(\lambda \mid \tau),
$$

on any vector of $\overline{\mathbb{D}}_{(6 \mathrm{VD}), \mathrm{N}}^{\mathcal{N}}$ and so it also follows:

$$
\mathrm{T}^{(8 \mathrm{~V})}(\lambda) \mathrm{S}_{q}^{\mathcal{R}} \overline{\mathbb{P}}_{\mathrm{N}}^{\mathcal{R}}=\mathrm{S}_{q}^{\mathcal{R}} \overline{\mathbb{P}}_{\mathrm{N}}^{\mathcal{R}} \overline{\mathcal{T}}^{(6 \mathrm{VD})}(\lambda \mid \tau)
$$

Proof. The identity (3.50) follows by computing the action on the generic elements of the dynamical-spin basis of $\overline{\mathbb{D}}_{(6 \mathrm{VD}), \mathrm{N}}^{\mathcal{R}}$ and using the orthonormality of these states.

Let us prove now (3.51), we use first the identities:

$$
\begin{aligned}
& \mathcal{C}(\lambda \mid \tau)=\mathrm{C}(\lambda \mid \tau) \mathrm{T}_{\tau}^{+}=\mathrm{T}_{\tau}^{+} \mathrm{C}(\lambda \mid \tau-\eta), \\
& \mathcal{B}(\lambda \mid \tau)=\mathrm{B}(\lambda \mid \tau) \mathrm{T}_{\tau}^{-}=\mathrm{T}_{\tau}^{-} \mathrm{B}(\lambda \mid \tau+\eta),
\end{aligned}
$$

to write:

$$
S_{q}(\tau-\eta) \mathrm{C}(\lambda \mid \tau-\eta)=\mathrm{T}_{\tau}^{-} S_{q}(\tau) \mathcal{C}(\lambda \mid \tau), \quad S_{q}(\tau+\eta) \mathrm{B}(\lambda \mid \tau+\eta)=\mathrm{T}_{\tau}^{+} \mathcal{B}(\lambda \mid \tau) S_{q}(\tau) .
$$

Then the following identities hold:

$$
\begin{aligned}
\overline{\mathbb{P}}_{\mathrm{N}}^{\mathcal{R}} \mathrm{T}_{\tau}^{-} S_{q}(\tau) \mathcal{C}(\lambda \mid \tau)\left|h_{1}, \ldots, h_{\mathrm{N}}\right\rangle & =\sum_{\mathrm{s}=-\mathrm{N}}^{\mathrm{N}}\left\langle t(\mathbf{s})-\eta\left|S_{q}\left(t_{\mathbf{h}}-\eta\right) \mathcal{C}\left(\lambda \mid t_{\mathbf{h}}\right)\right| h_{1}, \ldots, h_{\mathrm{N}}\right\rangle \\
& =\sum_{\mathrm{s}=-\mathrm{N}}^{\mathrm{N}}\left\langle t(\mathrm{~s})\left|S_{q}\left(t_{\mathbf{h}}-\eta\right) \mathcal{C}\left(\lambda \mid t_{\mathbf{h}}\right)\right| h_{1}, \ldots, h_{\mathrm{N}}\right\rangle \\
& =\overline{\mathbb{P}}_{\mathrm{N}}^{\mathcal{R}} S_{q}(\tau) \mathcal{C}(\lambda \mid \tau)\left|h_{1}, \ldots, h_{\mathrm{N}}\right\rangle \\
& =\left\langle h_{1}, \ldots, h_{\mathrm{N}}\right| \mathcal{C}(\lambda \mid \tau) \overline{\mathbb{P}}_{\mathbf{N}}^{\mathcal{L}}\left[\mathbf{S}_{q}^{\mathcal{L}}\right]^{-1},
\end{aligned}
$$


where (3.57) is equal to (3.56) being $\mathcal{C}(\lambda \mid \tau)\left|h_{1}=0, \ldots, h_{\mathrm{N}}=0\right\rangle$ zero while the last equality follows directly by (3.50) being $\mathcal{C}(\lambda \mid \tau)\left|h_{1}, \ldots, h_{\mathrm{N}}\right\rangle \in \overline{\mathbb{D}}_{(6 \mathrm{VD}), \mathrm{N}}^{\mathcal{R}}$; similarly one can prove the second identity in (3.51).

The identity (3.52) then is proven by the following set of equalities which hold on $\overline{\mathbb{D}}_{(6 \mathrm{VD}), \mathrm{N}}^{\mathcal{R}}$ :

$$
\begin{aligned}
& \mathrm{T}^{(8 \mathrm{~V})}(\lambda) \mathrm{S}_{q}^{\mathcal{R}} \overline{\mathbb{P}}_{\mathrm{N}}^{\mathcal{R}}=\overline{\mathbb{P}}_{\mathrm{N}}^{\mathcal{R}} \mathrm{T}^{(8 \mathrm{~V})}(\lambda) S_{q}(\tau) \\
& =\overline{\mathbb{P}}_{\mathrm{N}}^{\mathcal{R}} S_{q}(\tau-\eta) \mathrm{C}(\lambda \mid \tau-\eta)+\overline{\mathbb{P}}_{\mathrm{N}}^{\mathcal{R}} S_{q}(\tau+\eta) \mathrm{B}(\lambda \mid \tau+\eta) \\
& (3.50) \\
& =\mathrm{S}_{q}^{\mathcal{R}} \overline{\mathbb{P}}_{\mathrm{N}}^{\mathcal{R}} \overline{\mathcal{T}}^{(6 \mathrm{VD})}(\lambda \mid \tau) .
\end{aligned}
$$

\subsubsection{On the periodic 8-vertex transfer matrix spectrum by SOV in odd chains}

It is central to remark that the operator $S_{q}^{\mathcal{R}} \in \operatorname{End}\left(\mathbb{S}_{N}^{\mathcal{R}}\right)$ is not invertible in $\mathbb{S}_{N}^{\mathcal{R}}$. This statement is simply verified observing that the subspace of $\mathbb{S}_{N}^{\mathcal{R}}$ generated by the vectors:

$$
\otimes_{n=1}^{\mathrm{N}-1}\left|n, h_{n}\right\rangle \otimes\left(\left|\mathrm{N}, h_{\mathrm{N}}=1\right\rangle-\left|\mathrm{N}, h_{\mathrm{N}}=0\right\rangle\right) \text { for which } \sum_{a=1}^{\mathrm{N}-1} h_{a}=\frac{\mathrm{N}-1}{2},
$$

belongs to the kernel of $\mathrm{S}_{q}^{\mathcal{R}}$ as all these states are clearly annihilated by the action of the operator $S_{\mathrm{N}}\left(\xi_{\mathrm{N}} \mid \frac{\eta}{2} \sum_{a=1}^{\mathrm{N}-1} \sigma_{a}^{z-}\right.$ $\frac{\eta}{2} \sigma_{\mathrm{N}}^{z}$ ). Then, it is clear that we cannot use the identity (3.52) to completely reconstruct the spectrum of the periodic 8 -vertex transfer matrix $\mathrm{T}^{(8 \mathrm{~V})}(\lambda)$ by using the SOV characterization of the spectrum of the antiperiodic dynamical 6-vertex transfer matrix $\overline{\mathcal{T}}^{(6 \mathrm{VD})}(\lambda \mid \tau)$. Nevertheless, we can use (3.52) to define a first criterion to select eigenvalues of $\overline{\mathcal{T}}^{(6 \mathrm{VD})}(\lambda \mid \tau)$ which are also eigenvalues of $\mathrm{T}^{(8 \mathrm{~V})}(\lambda)$ and to associate to anyone of these eigenvalues just one eigenvector of $\mathrm{T}^{(8 \mathrm{~V})}(\lambda)$. In particular, we can prove the following lemma:

Lemma 3.4. Let us consider a $\mathrm{t}_{6 \mathrm{VD}}(\lambda) \in \Sigma_{\overline{\mathcal{T}}^{(6 \mathrm{VD})}}$ and let $\left|\mathrm{t}_{6 \mathrm{VD}}\right\rangle$ be the corresponding eigenvector of $\overline{\mathcal{T}}^{(6 \mathrm{VD})}(\lambda \mid \tau)$, then if the vector

$$
\overline{\mathbb{P}}_{\mathrm{N}}^{\mathcal{R}}\left|\mathrm{t}_{6 \mathrm{VD}}\right\rangle=\sum_{h_{1}, \ldots, h_{\mathrm{N}}=0}^{1} \prod_{a=1}^{\mathrm{N}} Q_{\mathrm{t}}\left(\xi_{a}^{\left(h_{a}\right)}\right) \operatorname{det}_{\mathrm{N}} \Theta_{i j}^{(\boldsymbol{h})} \underline{\left|h_{1}, \ldots, h_{\mathrm{N}}\right\rangle}, \text { with } \underline{\left|h_{1}, \ldots, h_{\mathrm{N}}\right\rangle}=\left\langle t_{\boldsymbol{h}} \mid h_{1}, \ldots, h_{\mathrm{N}}\right\rangle
$$

does not belong to the kernel of $\mathrm{S}_{q}^{\mathcal{R}}, \mathrm{t}_{6 \mathrm{VD}}(\lambda)$ is eigenvalue of $\mathrm{T}^{(8 \mathrm{~V})}(\lambda)$ and $\mathrm{S}_{q}^{\mathcal{R}} \overline{\mathbb{P}}_{\mathrm{N}}^{\mathcal{R}}\left|\mathrm{t}_{6 \mathrm{VD}}\right\rangle$ is one corresponding eigenvector.

Proof. Under the condition:

$$
\mathrm{S}_{q}^{\mathcal{R}} \overline{\mathbb{P}}_{\mathrm{N}}^{\mathcal{R}}\left|\mathrm{t}_{6 \mathrm{VD}}\right\rangle \neq \underline{0} \in \mathbb{S}_{\mathrm{N}}^{\mathcal{R}}
$$

the proposition is a simple consequence of the identity (3.52); indeed, it hold:

$$
\mathrm{T}^{(8 \mathrm{~V})}(\lambda) \mathrm{S}_{q}^{\mathcal{R}} \overline{\mathbb{P}}_{\mathrm{N}}^{\mathcal{R}}\left|\mathrm{t}_{6 V D}\right\rangle=\mathrm{S}_{q}^{\mathcal{R}} \overline{\mathbb{P}}_{\mathrm{N}}^{\mathcal{R}} \overline{\mathcal{T}}^{(6 \mathrm{VD})}(\lambda \mid \tau)\left|\mathrm{t}_{6 V D}\right\rangle=\mathrm{S}_{q}^{\mathcal{R}} \overline{\mathbb{P}}_{\mathrm{N}}^{\mathcal{R}}\left|\mathrm{t}_{6 \mathrm{VD}}\right\rangle \mathrm{t}_{6 \mathrm{VD}}(\lambda)
$$


Remark 2. It is worth remarking that we need this criterion as, from the results in subsection 3.3, we only know that the set $\Sigma_{\mathrm{T}^{(8 \mathrm{~V})}}$ of the eigenvalues of $\mathrm{T}^{\left({ }^{(8)}\right)}(\lambda)$ is contained in the set $\Sigma_{\overline{\mathcal{T}}^{(6 \mathrm{VD})}}$ of the eigenvalues of $\overline{\mathcal{T}}^{(6 \mathrm{VD})}(\lambda \mid \tau)$ in the case of a chain with an odd number of quantum sites. Moreover, it is important to clarify that currently we have only proven that the above lemma defines a criterion, i.e. a sufficient condition for an element of $\Sigma_{\overline{\mathcal{T}}^{(6 \mathrm{VD})}}$ to be also an element of $\Sigma_{\mathbf{T}(8 \mathrm{~V})}$. It will be fundamental to understand if this is also a necessary condition as in this last case we will get a complete characterization of $\Sigma_{\mathrm{T}^{(8 \mathrm{~V})}}$ and one eigenstate of $\mathrm{T}^{(8 \mathrm{~V})}(\lambda)$ for any element of $\Sigma_{\mathrm{T}^{(8 \mathrm{~V})}}$ just using the SOV characterization of the spectrum of $\overline{\mathcal{T}}^{(6 \mathrm{VD})}(\lambda \mid \tau)$. Finally, let us point out that the fact that $\mathrm{S}_{q}^{\mathcal{R}} \in \operatorname{End}\left(\mathbb{S}_{\mathrm{N}}^{\mathcal{R}}\right)$ is not invertible in $\mathbb{S}_{N}^{\mathcal{R}}$ is just required to make the identity (3.52) compatible with the observed degeneracy of the spectrum of $\mathrm{T}^{(8 \mathrm{~V})}(\lambda)$ for the cases $\mathrm{N}=1$ and 3 explicitly analyzed in appendix. As in presence of this degeneracy

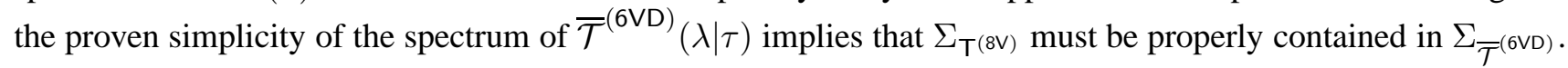

\section{Conclusion}

In this paper we have focused our attention on the highest weight representations of the dynamical 6-vertex YangBaxter algebra on a generic spin-1/2 quantum chain with an odd number $\mathrm{N}$ of sites. We have studied the integrable quantum model associated to the antiperiodic boundary conditions in the framework of the SOV method. For this integrable quantum models, we have derived:

- The complete SOV description of transfer matrix eigenvalues and eigenstates and the simplicity of the spectrum.

- Matrix elements of the identity on separate states expressed by one determinant formulae of $\mathrm{N} \times \mathrm{N}$ matrices with elements given by sums over the eigenvalues of the quantum separate variables of the product of the coefficients of the left/right separate states, which holds in particular for the eigenstates of antiperiodic dynamical 6-vertex transfer matrix.

The results derived in this paper provide the required setup to compute matrix elements on transfer matrix eigenstates of local operators. The analysis of the following steps:

- local operator reconstructions in terms of Sklyanin's quantum separate variables,

- form factors of the local operators on the transfer matrix eigenstates in determinant form,

will be presented in a paper which is currently under completion in collaboration with Levy-Bencheton and Terras [160]. The study of correlation functions done in [161] for the periodic dynamical 6-vertex chain even if developed in the framework of the algebraic Bethe ansatz is relevant also for the current analysis. Indeed, the reconstruction of local operators of [161] can be adapted to the antiperiodic dynamical 6-vertex chain to get reconstruction of local operators in terms of the quantum separate variables. Then the computation of the form factors for the antiperiodic dynamical 6-vertex proceed in a similar way to that of the standard 6-vertex quantum chain with antiperiodic boundary conditions as derived in [3] in the SOV framework. It is also worth mentioning that the knowledge of the form factors of local operators represents also an efficient tools for controlled numerical analysis of correlation functions. Indeed, the decomposition of the identity (2.133) allows to rewrite the correlation functions in terms of form factors and then it is a priori possible to apply the same kind of approach developed in [162] in the ABA framework 27 also in our SOV framework to get numerical evaluations of correlation functions.

\footnotetext{
${ }^{27}$ See the series of papers [162]-[168] where the dynamical structure factors, observable by neutron scattering experiments [169]-[175], were numerically evaluated.
} 
We have moreover shown that the existence of gauge transformations allows us to use the antiperiodic dynamical 6-vertex transfer matrix as a tool to further analyze the spectral problem of the periodic 8-vertex transfer matrix in the case of a chain with $\mathrm{N}$ odd and for general values of the coupling constant $\eta$ (non restricted to the elliptic roots of unit). The potential relevance of this analysis is made clear observing that the standard Bethe ansatz analysis developed in [23, 22, 24, 8] does not apply to this case. More in detail, we have shown that the gauge transformations allow to define a criterion to select eigenvalues of the antiperiodic dynamical 6-vertex transfer matrix which are also eigenvalues of the periodic 8-vertex transfer matrix, moreover associating to any one of them one nonzero periodic 8vertex eigenstate. Finally, let us stress the importance to understand if this criterion also define a necessary condition for a antiperiodic dynamical 6-vertex eigenvalue to be also a periodic 8-vertex eigenvalue. Indeed, in this case the SOV characterization of the antiperiodic dynamical 6-vertex spectrum will also allow the complete characterization of the periodic 8-vertex eigenvalues and the construction of one of its eigenstates for anyone of its eigenvalues. The answer to this fundamental question requires a systematic and simultaneous analysis of the degeneracy of the periodic 8-vertex spectrum and of the dimension of the kernel of the operator $S_{q}^{\mathcal{R}}$ which we will try to address elsewhere.

Acknowledgments The author gratefully acknowledge B. McCoy for the many stimulating discussions on the 8vertex model and the interesting questions on quantum separation of variables which have strongly inspired and motivated the author to develop the present paper. The author would also like to thank N. Kitanine, K. K. Kozlowski and J. M. Maillet for their interest and D. Levy-Bencheton and V. Terras for their interest, attentive reading and remarks on a first draft of this paper. The author is supported by National Science Foundation grants PHY-0969739 and gratefully acknowledges the YITP Institute of Stony Brook for the opportunity to develop his research programs. The author would also like to thank for their hospitality the Theoretical Physics Group of the Laboratory of Physics at ENS-Lyon and the Mathematical Physics Group at IMB of the Dijon University (under support ANR-10-BLAN0120-04-DIADEMS).

\section{A Appendix}

Here we analyze explicitly the spectral problem of the transfer matrices $\mathrm{T}^{(8 \mathrm{~V})}(\lambda)$ and $\overline{\mathcal{T}}^{(6 \mathrm{VD})}(\lambda \mid \tau)$ for the trivial cases of chains with $\mathrm{N}=1$ and $\mathrm{N}=3$. The aim is to make clear some of the above statements and to have some basic analysis about the degeneracy of the $\mathrm{T}^{(8 \mathrm{~V})}(\lambda)$ spectrum.

\section{A.1 Spectrum of $\mathrm{T}^{(8 \mathrm{~V})}(\lambda)$ and $\overline{\mathcal{T}}^{(6 \mathrm{VD})}(\lambda \mid \tau)$ for $\mathrm{N}=1$}

In the one site case it holds:

$$
\mathrm{T}^{(8 \mathrm{~V})}(\lambda)=(\mathrm{a}(\lambda)+\mathrm{b}(\lambda))\left(\begin{array}{ll}
1 & 0 \\
0 & 1
\end{array}\right) \text { on } \mathbb{S}_{\mathrm{N}=1}^{\mathcal{R}},
$$

and from:

$$
\overline{\mathcal{T}}^{(6 \mathrm{VD})}(\lambda \mid \tau)=\mathrm{T}_{\tau}^{+} \mathrm{C}(\lambda \mid \tau-\eta)+\mathrm{T}_{\tau}^{-} \mathrm{B}(\lambda \mid \tau+\eta)
$$

it holds

$$
\overline{\mathcal{T}}^{(6 \mathrm{VD})}(\lambda \mid \tau)=c(\lambda \mid \eta / 2)\left(\begin{array}{cc}
0 & \mathrm{~T}_{\tau}^{+} \\
\mathrm{T}_{\tau}^{-} & 0
\end{array}\right) \text { on } \mathbb{D}_{(6 \mathrm{VD}), \mathrm{N}=1}^{\mathcal{R}}
$$


Then, we have that the spectrum of $\overline{\mathcal{T}}^{(6 \mathrm{VD})}(\lambda \mid \tau)$ is simple and characterized by:

$$
\overline{\mathcal{T}}^{(6 \mathrm{VD})}(\lambda \mid \tau)\left|\mathrm{t}_{6 \mathrm{VD}}^{( \pm)}\right\rangle=\left|\mathrm{t}_{6 \mathrm{VD}}^{( \pm)}\right\rangle \mathrm{t}_{6 \mathrm{VD}}^{( \pm)}(\lambda)
$$

where we have defined:

$$
\mathrm{t}_{6 \mathrm{VD}}^{( \pm)}(\lambda) \equiv \pm c(\lambda \mid \eta / 2),\left|\mathrm{t}_{6 \mathrm{VD}}^{( \pm)}\right\rangle \equiv\left(\begin{array}{c} 
\pm|t(1)\rangle \\
|t(-1)\rangle
\end{array}\right) \in \mathbb{D}_{(6 \mathrm{VD}), \mathrm{N}=1}^{\mathcal{R}},
$$

and $|t(a)\rangle$ is the $\tau$ eigenstate with eigenvalue $t(a) \equiv-\eta a / 2$. Instead, the spectrum of $\mathrm{T}^{(8 \mathrm{~V})}(\lambda)$ is double degenerate with eigenvalue $\operatorname{t}_{8 \mathrm{v}}(\lambda)=\mathrm{a}(\lambda)+\mathrm{b}(\lambda)$, then the identity:

$$
\mathrm{a}(\lambda)+\mathrm{b}(\lambda)=c(\lambda \mid \eta / 2)
$$

implies the proper set inclusion $\Sigma_{\mathbf{T}^{(8 \mathrm{~V})}} \subset \Sigma_{\overline{\mathcal{T}}^{(6 \mathrm{VD})}}$ for the $\mathrm{N}=1$ case. Let us now observe that:

$$
\mathbf{S}_{q}^{\mathcal{R}}=S_{1}\left(\lambda \mid-\eta / 2 \sigma_{1}^{z}\right) \equiv\left(\begin{array}{cc}
\theta_{2}(\lambda+\eta / 2 \mid 2 w) & \theta_{2}(\lambda+\eta / 2 \mid 2 w) \\
\theta_{3}(\lambda+\eta / 2 \mid 2 w) & \theta_{3}(\lambda+\eta / 2 \mid 2 w)
\end{array}\right)_{0}
$$

and:

$$
\mathrm{S}_{q}^{\mathcal{R}} \overline{\mathbb{P}}_{\mathrm{N}=1}^{\mathcal{R}}\left|\mathbf{t}_{\mathrm{6VD}}^{( \pm)}\right\rangle=\left(\begin{array}{c}
\theta_{2}(\lambda+\eta / 2 \mid 2 w)(1 \pm 1) \\
\theta_{3}(\lambda+\eta / 2 \mid 2 w)(1 \pm 1)
\end{array}\right) \in \mathbb{S}_{\mathrm{N}}^{\mathcal{R}}
$$

Then, in agreement with the Lemma 3.4, we have that $\mathrm{t}_{6 \mathrm{VD}}^{(+)}(\lambda)$ is $\mathrm{T}^{(8 \mathrm{~V})}(\lambda)$ eigenvalue and:

$$
\mathbf{S}_{q}^{\mathcal{R}} \overline{\mathbb{P}}_{\mathrm{N}=1}^{\mathcal{R}}\left|\mathrm{t}_{6 \mathrm{VD}}^{(+)}\right\rangle=2\left(\begin{array}{c}
\theta_{2}(\lambda+\eta / 2 \mid 2 w) \\
\theta_{3}(\lambda+\eta / 2 \mid 2 w)
\end{array}\right) \in \mathbb{S}_{\mathrm{N}}^{\mathcal{R}}
$$

is one corresponding eigenstate. It is also interesting to remark that the eigenvalue $\mathrm{t}_{6 \mathrm{VD}}^{(-)}(\lambda)$ of $\overline{\mathcal{T}}^{(6 \mathrm{VD})}(\lambda \mid \tau)$ for which it holds $\mathrm{S}_{q}^{\mathcal{R}} \overline{\mathbb{P}}_{\mathrm{N}=1}^{\mathcal{R}}\left|\mathrm{t}_{6 \mathrm{VD}}^{(-)}\right\rangle=\underline{0}$ is not an eigenvalue of $\mathrm{T}^{(8 \mathrm{~V})}(\lambda)$.

\section{A.2 Spectrum of $\mathrm{T}^{(8 \mathrm{~V})}(\lambda)$ and $\overline{\mathcal{T}}^{(6 \mathrm{VD})}(\lambda \mid \tau)$ for $\mathrm{N}=3$}

\section{A.2.1 General statements on the spectrum for $N=3$}

The system of equations which completely characterize the spectrum (eigenvalues and eigenvectors) of $\overline{\mathcal{T}}^{(6 \mathrm{VD})}(\lambda \mid \tau)$ reads:

$$
x_{n}\left(\sum_{a=1}^{\mathrm{N}} J_{n, a} x_{a}\right)-q_{n}=0 \quad \forall n \in\{1, \ldots, \mathrm{N}\} .
$$

It is an inhomogeneous system of $\mathrm{N}$ quadratic equations in the $\mathrm{N}$ unknown $x_{n}=\mathrm{t}_{6 \mathrm{VD}}\left(\xi_{n}\right)$ with coefficients characterized by:

$$
J_{n, a}=\frac{\theta\left(t_{\mathbf{0}}-\xi_{n}+\xi_{a}+\eta\right)}{\theta\left(t_{\mathbf{0}}\right)} \prod_{b \neq a} \frac{\theta\left(\xi_{n}-\xi_{b}-\eta\right)}{\theta\left(\xi_{a}-\xi_{b}\right)}, \quad q_{n}=\mathrm{A}\left(\xi_{n}^{(0)}\right) \mathrm{D}\left(\xi_{n}^{(1)}\right), \quad \forall n \in\{1, \ldots, \mathrm{N}\},
$$

as it is simple to derive substituting in (2.103) the interpolation formula (2.104) for $\mathrm{t}_{6 \mathrm{VD}}\left(\xi_{n}^{(1)}\right)$. It is trivial to observe that the set of the solutions to this system has a $Z_{2}$ symmetry; i.e. if $\mathrm{z}_{\mathrm{N}}^{(+)} \equiv\left\{x_{1}, \ldots, x_{n}, \ldots, x_{\mathrm{N}}\right\}$ is a solution of it 
then also $\mathrm{z}_{\mathrm{N}}^{(-)} \equiv\left\{-x_{1}, \ldots,-x_{n}, \ldots,-x_{\mathrm{N}}\right\}$ is a solution. So from the interpolation formula (2.104), it follows that if $\mathrm{t}_{6 \mathrm{VD}}^{(+)}(\lambda) \in \Sigma_{\overline{\mathcal{T}}^{(6 \mathrm{VD})}}$ then also $\mathrm{t}_{6 \mathrm{VD}}^{(-)}(\lambda)=\left(-\mathrm{t}_{6 \mathrm{VD}}^{(+)}(\lambda)\right) \in \Sigma_{\overline{\mathcal{T}}^{(6 \mathrm{VD})}}$.

In the case $N=3$, we have verified that the system (A.10) has $2^{3}$ distinct solutions. Then $\Sigma_{\overline{\mathcal{T}}^{(6 \mathrm{VD})}}$ is composed by $2^{3}$ distinct elliptic polynomials $\mathrm{t}_{6 \mathrm{VD}}^{( \pm, a)}(\lambda)$ with $a \in\{1, \ldots, 4\}$ and so $\overline{\mathcal{T}}^{(6 \mathrm{VD})}(\lambda \mid \tau)$ has simple spectrum as proven in this paper. Moreover, in the case of $\mathrm{N}=3$, we have studied the spectrum of $\mathrm{T}^{(8 \mathrm{~V})}(\lambda)$ and we have observed that it has 4 distinct eigenvalues each one being double degenerate. We have observed that the set $\Sigma_{\mathbf{T}^{(8 \mathrm{~V})}} \equiv\left\{\mathrm{t}_{\mathrm{8V}}^{(1)}(\lambda), \mathrm{t}_{8 \mathrm{~V}}^{(2)}(\lambda), \mathrm{t}_{\mathrm{8V}}^{(3)}(\lambda), \mathrm{t}_{8 \mathrm{~V}}^{(4)}(\lambda)\right\}$ coincide with the set of elliptic polynomials which are generated by using 4 distinct solutions $z_{\mathrm{N}}^{(a)}$ of the system (A.10) and the interpolation formula (2.104). Moreover, the solutions $z_{\mathrm{N}}^{(a)}$ with $a \in\{1, \ldots, 4\}$ used to construct $\Sigma_{\mathrm{T}^{(8 \mathrm{~V})}}$ appear not to be related by the $Z_{2}$ symmetry of the system (A.10); in fact, it holds:

$$
z_{\mathrm{N}}^{(a)} \neq-z_{\mathrm{N}}^{(b)} \text { for any } a, b \in\{1, \ldots, 4\}
$$

Let us fix the notation:

$$
\mathrm{t}_{6 \mathrm{VD}}^{( \pm, a)}(\lambda) \equiv \pm \mathrm{t}_{8 \mathrm{~V}}^{(a)}(\lambda) \text { for any } a \in\{1, \ldots, 4\}
$$

then we can summarize the above observations on the spectrum of $\overline{\mathcal{T}}^{(6 \mathrm{VD})}(\lambda \mid \tau)$ and $\mathrm{T}^{(8 \mathrm{~V})}(\lambda)$ by saying that to the two nondegenerate eigenvalues $\mathrm{t}_{6 \mathrm{VD}}^{(+, a)}(\lambda)$ and $\mathrm{t}_{6 \mathrm{VD}}^{(-, a)}(\lambda)$ of $\overline{\mathcal{T}}^{(6 \mathrm{VD})}(\lambda \mid \tau)$ it is associates just the double degenerate eigenvalue $\mathrm{t}_{8 \mathrm{~V}}^{(a)}(\lambda)$ of $\mathrm{T}^{(8 \mathrm{~V})}(\lambda)$. Our analysis shows that this statement holds for $\mathrm{N}=1$ and $\mathrm{N}=3$ to be able to verify if it persists for a generic odd $\mathrm{N}$ can be one central step toward the characterization of the spectrum of $\mathrm{T}^{(8 \mathrm{~V})}(\lambda)$ in terms of the SOV characterization of the $\overline{\mathcal{T}}^{(6 \mathrm{VD})}(\lambda \mid \tau)$ spectrum.

\section{A.2.2 Some numerical data for $\mathrm{N}=3$}

For convenience of the reader, we write explicitly the periodic 8 -vertex transfer matrix $\mathrm{T}^{(8 \mathrm{~V})}(\lambda)$ in the $\mathrm{N}=3$ case:

$$
\left(\begin{array}{c}
\mathrm{a}_{1} \mathrm{a}_{2} \mathrm{a}_{3}+\mathrm{b}_{1} \mathrm{~b}_{2} \mathrm{~b}_{3} \\
0 \\
0 \\
\mathrm{a}_{1} \mathrm{~d}_{2} \mathrm{c}_{3}+\mathrm{b}_{1} \mathrm{c}_{2} \mathrm{~d}_{3} \\
0 \\
\mathrm{~d}_{1} \mathrm{~b}_{2} \mathrm{c}_{3}+\mathrm{c}_{1} \mathrm{a}_{2} \mathrm{~d}_{3} \\
\mathrm{~d}_{1} \mathrm{c}_{2} \mathrm{a}_{3}+\mathrm{c}_{1} \mathrm{~d}_{2} \mathrm{~b}_{3} \\
0
\end{array}\right.
$$
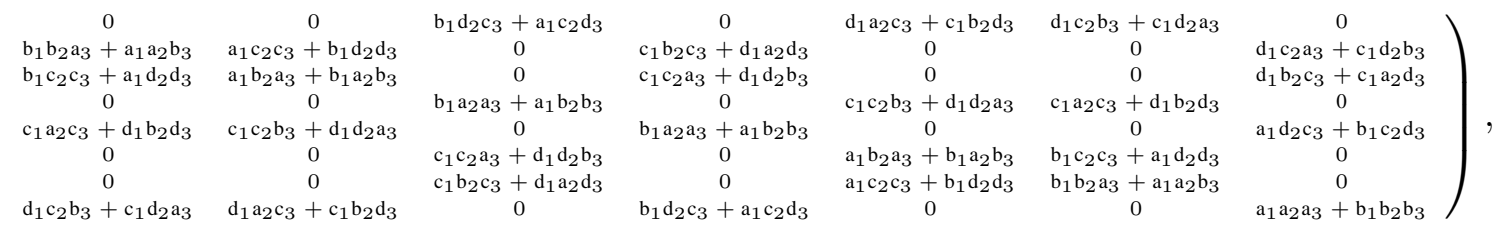

where we have used the notations:

$$
\mathrm{a}_{n}=\mathrm{a}\left(\lambda-\xi_{n}\right), \mathrm{b}_{n}=\mathrm{b}\left(\lambda-\xi_{n}\right), \mathrm{c}_{n}=\mathrm{c}\left(\lambda-\xi_{n}\right), \mathrm{d}_{n}=\mathrm{d}\left(\lambda-\xi_{n}\right)
$$

To verify the statements done in the previous subsection it is only need to write in Mathematica the previous $8 \times 8$ matrix and the system of equations (A.10) for $\mathrm{N}=3$ and solve the eigenvalue problem and the system for generic values of the 5 parameters $\left(\xi_{1}, \xi_{2}, \xi_{3}, \eta, t=e^{i \pi w}\right)$. Here we report just few numerical data as a confirmation that we have really implemented this numerical exercise. Defined $\mathrm{w}_{3}^{(a)}=\left\{\mathrm{t}_{8 \mathrm{~V}}^{(a)}\left(\xi_{1}\right), \mathrm{t}_{8 \mathrm{~V}}^{(a)}\left(\xi_{2}\right), \mathrm{t}_{8 \mathrm{~V}}^{(a)}\left(\xi_{3}\right)\right\}$, it holds: 
1) For $\xi_{1}=5.7, \xi_{2}=1.5, \xi_{3}=0.22, \eta=0.7, t=0.26$ :

$$
\begin{aligned}
& \mathrm{z}_{3}^{( \pm, 1)}= \pm\{2.4648971133384494,0.5263660613291964,-0.0461646762536026\} \\
& \mathrm{z}_{3}^{( \pm, 2)}= \pm\{0.16746377944367666,0.09438584696000717,-3.7893847598813264\} \\
& \mathrm{z}_{3}^{( \pm, 3)}= \pm\{0.15697838428546823,0.5124574129431847,-0.7445585159876167\} \\
& \mathrm{z}_{3}^{( \pm, 4)}= \pm\{0.02568158650662899,3.433163601035112,-0.679328947667353\} \\
& \mathrm{w}_{3}^{(1)}=\{2.46489711333845,0.5263660613291976,-0.0461646762536022\} \\
& \mathrm{w}_{3}^{(2)}=\{0.167463779423851,0.0943858469664461,-3.789384759881333\} \\
& \mathrm{w}_{3}^{(3)}=\{0.15697838428547273,0.5124574129431814,-0.7445585159876165\} \\
& \mathrm{w}_{3}^{(4)}=\{0.025681586506630664,3.4331636010351154,-0.6793289476673527\} .
\end{aligned}
$$

2) For $\xi_{1}=2.5, \xi_{2}=3.1, \xi_{3}=1.33, \eta=0.3, t=0.45$ :

$$
\begin{aligned}
& \mathrm{z}_{3}^{( \pm, 1)}= \pm\{-2.3672052885387806,-0.03421683553328285,0.560404707906603\} \\
& \mathrm{z}_{3}^{( \pm, 2)}= \pm\{0.1607220217069632,7.959749585813279,0.03548156343430941\} \\
& \mathrm{z}_{3}^{( \pm, 3)}= \pm\{0.14344459641406113,0.5655603642746968,0.5595184106850913\} \\
& \mathrm{z}_{3}^{( \pm, 4)}= \pm\{0.009963704747040916,0.5039536632240319,9.03990912589408\} \\
& \mathrm{w}_{3}^{(1)}=\{-2.367205288523499,-0.034216835529656396,0.5604047079065965\} \\
& \mathrm{w}_{3}^{(2)}=\{0.1607220217069637,7.95974958581329,0.03548156343431045\} \\
& \mathrm{w}_{3}^{(3)}=\{0.14344459639912585,0.5655603642711194,0.5595184106850958\} \\
& \mathrm{w}_{3}^{(4)}=\{0.009963750993033916,0.5039536669291063,0.5595184106850958\} .
\end{aligned}
$$

3) For $\xi_{1}=1.7, \xi_{2}=3.5, \xi_{3}=5.22, \eta=4.7, t=0.05$ :

$$
\begin{aligned}
& \mathrm{z}_{3}^{( \pm, 1)}= \pm\{0.9071447507669119,0.0010355130798548361,-0.6163903868766624\} \\
& \mathrm{z}_{3}^{( \pm, 2)}= \pm\{-0.18602724783757033,-0.02888852650572982,-0.10774226124070294\} \\
& \mathrm{z}_{3}^{( \pm, 3)}= \pm\{0.13725423857934435,-0.024752594653532196,0.1704282336621456\} \\
& \mathrm{z}_{3}^{( \pm, 4)}= \pm\{-0.04740255397294748,0.8919753005921505,0.013694099141681645\} \\
& \mathrm{w}_{3}^{(1)}=\{0.907144750766913,0.001035513079898853,-0.6163903868766655\} \\
& \mathrm{w}_{3}^{(2)}=\{-0.18602724783757013,-0.028888526505732478,-0.10774226124070306\} \\
& \mathrm{w}_{3}^{(3)}=\{0.13725423857934346,-0.02475259465352673,0.17042823366214616\} \\
& \mathrm{w}_{3}^{(4)}=\{-0.04740255397294748,0.8919753005921487,0.013694099141681883\} .
\end{aligned}
$$

4) For $\xi_{1}=49.7, \xi_{2}=10.5, \xi_{3}=12.22, \eta=5.87, t=0.726$ :

$$
\begin{aligned}
& \mathrm{z}_{3}^{( \pm, 1)}= \pm\{0.158866785906656,-0.002317414600871322,0.004665001427754174\} \\
& \mathrm{z}_{3}^{( \pm, 2)}= \pm\{0.004163560745980359,-0.13352504997041553,0.0030893063326063934\} \\
& \mathrm{z}_{3}^{( \pm, 3)}= \pm\{0.0027572370077268236,-7.693461066977195,0.00008096415168424851\} \\
& \mathrm{z}_{3}^{( \pm, 4)}= \pm\{-0.001396539108516703,-0.13352504998006434,-0.009210278823835091\} \\
& \mathrm{w}_{3}^{(1)}=\{0.15886678590666517,-0.0023174146009546297,0.0046650014277542385\} \\
& \mathrm{w}_{3}^{(2)}=\{0.004163560745980381,-0.13352504997042003,0.003089306332606317\} \\
& \mathrm{w}_{3}^{(3)}=\{0.002757237007726877,-7.693461066977227,0.00008096415168424613\} \\
& \mathrm{w}_{3}^{(4)}=\{-0.001396539108516455,-0.133525049979987,-0.009210278823835037\} .
\end{aligned}
$$


5) For $\xi_{1}=11.2, \xi_{2}=1.1, \xi_{3}=0.82, \eta=3.3, t=0.096$ :

$$
\begin{aligned}
& \mathrm{z}_{3}^{( \pm, 1)}= \pm\{-0.13845098667904934,-0.04279356398629822,0.017867992946492404\} \\
& \mathrm{z}_{3}^{( \pm, 2)}= \pm\{0.12350539448737866,0.022662651149136445,0.03782279719611843\} \\
& \mathrm{z}_{3}^{( \pm, 3)}= \pm\{0.11482851797211138,-0.02822854036213841,-0.032659693368688764\} \\
& \mathrm{z}_{3}^{( \pm, 4)}= \pm\{-0.10167300872962227,0.052191832632450655,-0.019949961538809933\} \\
& \mathrm{w}_{3}^{(1)}=\{-0.13845098667905043,-0.04279356398629837,0.01786799294649241\} \\
& \mathrm{w}_{3}^{(2)}=\{0.1235053944873589,0.022662651149137868,0.03782279719611853\} \\
& \mathrm{w}_{3}^{(3)}=\{0.11482851797211588,-0.02822854036213898,-0.032659693368688944\} \\
& \mathrm{w}_{3}^{(4)}=\{-0.10167300872962239,0.05219183263245088,-0.019949961538809936\} .
\end{aligned}
$$

In all these cases (up to small numerical errors) it is possible to observe that any eigenvalue of $\mathrm{T}^{(8 \mathrm{~V})}(\lambda)$ is double degenerate and that the following identities hold:

$$
\mathrm{z}_{3}^{(+, a)}=\mathrm{w}_{3}^{(a)} \text { for any } a \in\{1, \ldots, 4\} .
$$

\section{References}

[1] N. Grosjean, J. M. Maillet, G. Niccoli, On form factors of local operators in the lattice sine-Gordon model, J. Stat. Mech. (2012) P10006.

[2] N. Grosjean, J. M. Maillet, G. Niccoli, On form factors of local operators in the $\tau_{2}$-model and the chiral Potts model, to appear.

[3] G. Niccoli, Antiperiodic spin-1/2 XXZ quantum chains by separation of variables: Form factors and complete spectrum, arXiv: 1205.4537 .

[4] G. Niccoli, Form factors and complete spectrum of XXX antiperiodic higher spin chains by quantum separation of variables, arXiv:1206.2418

[5] G. Niccoli, Non-diagonal open spin-1/2 XXZ quantum chain by separation of variables: Complete spectrum and matrix elements of some quasi-local operators, J. Stat. Mech. (2012) P11005.

[6] E. K. Sklyanin and L. D. Faddeev, Sov. Phys. Dokl. 23 (1978) 902.

[7] E. K. Sklyanin and L. A. Takhtajan, L. D. Faddeev, Theor. Math. Phys. 40 (1980) 688.

[8] L. A. Takhtajan, L. D. Faddeev, Russ. Math. Surv. 34 : 5 (1979) 11.

[9] E. K. Sklyanin, Dokl. Akad. Nauk SSSR 244 (1979) 1337; Sov. Phys. Dokl. 24 (1979) 107.

[10] P. P. Kulish and E. K. Sklyanin, Phys. Lett. A 70 (1979) 461.

[11] L. D. Faddeev, Sov. Sci. Rev. Math. Cl (1980) 107.

[12] E. K. Sklyanin, J. Sov. Math. 19 (1982) 1546.

[13] L. D. Faddeev, Les Houches lectures of 1982, Elsevier Sci. Publ. 563 (1984).

[14] L. D. Faddeev, How Algebraic Bethe Ansatz works for integrable model, hep-th/9605187v1.

[15] M. Jimbo, Adv. Series in Math. Phys. 10, Singapore, World Scientific, (1990).

[16] P. P. Kulish and E. K. Sklyanin, Lect. Notes in Phys. 151 (1982) 61.

[17] B. S. Shastry, Lect. Notes in Phys. 242 (1985).

[18] H. B. Thacker, Rev. Mod. Phys. 53 (1982) 253.

[19] A. G. Izergin and V. E. Korepin, Nucl. Phys. B 205 (1982) 401.

[20] G. Felder. Elliptic Quantum Groups. In Xlth International Congress of Mathematical Physics, Paris 1994 (D- Ialgonitzer, ed.), International Press (1995) 211.

[21] G. Felder and A. Varchenko, Comm. Math. Phys. 181 (1996) 741.

[22] R. J. Baxter, Ann. Phys. 76 (1973) 25.

[23] R. J. Baxter, Ann. Phys. 76 (1973) 1.

[24] R. J. Baxter, Ann. Phys. 76 (1973) 48. 
[25] G. Felder and A. Varchenko, Nucl. Phys. B 480 (1996) 485.

[26] M. Jimbo, T. Miwa, M. Okado, Comm. Math. Phys. 116 (1988) 507.

[27] W. Heisenberg, Z. Phys. 49 (1928) 619.

[28] H. Bethe, 1931 Z. Phys. 71205.

[29] L. Hulthen, Ark. Mat. Astron. Fys. 26 (1938) 1.

[30] R. Orbach, Phys. Rev. 112 (1958) 309.

[31] L. R. Walker, Phys. Rev. 116 (1959) 1089.

[32] C. N. Yang and C. P. Yang, Phys. Rev. 150 (1966) 321.

[33] C. N. Yang and C. P. Yang, Phys. Rev. 150 (1966) 327.

[34] M. Gaudin, La Fonction d'onde de Bethe, Paris: Masson (1983).

[35] E. H. Lieb and D. C. Mattis, Mathematical Physics in One Dimension, New-York: Academic (1966).

[36] R. J. Baxter, Ann. Phys. 70 (1972) 193.

[37] K. Fabricius and B. M. McCoy, J. Stat. Phys. 111 (2003) 323.

[38] K. Fabricius and B. M. McCoy, Functional equations and fusion matrices for the eight vertex model, Publ. RIMS, Kyoto Univ. 40 (2004) 905.

[39] K. Fabricius and B. M. McCoy, J. Stat. Phys. 120 (2005) 37.

[40] K. Fabricius, J. Phys. A 40 (2007) 4075.

[41] K. Fabricius, and B. M. McCoy, J. Phys. A 40 (2007) 14893.

[42] K. Fabricius, B. M. McCoy, J. Stat. Phys. 134 (2009) 643.

[43] R. J. Baxter, J. Statist. Phys. 108 (2002) 1.

[44] N. A. Slavnov, Theor. Math. Phys. 79 (1989) 502.

[45] N. A. Slavnov, Zap. Nauchn. Semin. POMI 245 (1997) 270.

[46] N. Kitanine, J. M. Maillet, and V. Terras, Nucl. Phys. B 554 (1999) 647.

[47] E. K. Sklyanin, Lect. Notes Phys. 226 (1985) 196; E. K. Sklyanin J. Sov. Math. 31 (1985) 3417.

[48] E. K. Sklyanin, Quantum inverse scattering method. Selected topics. In: Quantum groups and quantum integrable systems (World Scientific, 1992) 63, arXiv:hep-th/9211111v1.

[49] E. K. Sklyanin, Prog. Theor. Phys. Suppl. 118 (1995) 35.

[50] E. Date, M. Jimbo, A. Kuniba, T. Miwa, M. Okado. Exactly Solvable SOS-Models II: Proof of the Star-Triangle-Relation and Combinatorial Identities, in Adv. Stud. in Pure Math. 16 M. Jimbo, T. Miwa, A. Tsuchiya ed. Academic Press (1988) 17.

[51] G. Felder and A. Schorr, J. Phys. A: Math. Gen. 32 (1999) 8001.

[52] A. Schorr, Separation of variables for the eight-vertex SOS model with antiperiodic boundary conditions, http://e-collection.library.ethz.ch/eserv/eth:23538/eth-23538-02.pdf.

[53] J. M. Maillet and V. Terras, Nucl. Phys. B 575 (2000) 627.

[54] A. G. Izergin, N. Kitanine, J. M. Maillet, and V. Terras, Nucl. Phys. B 554 (1999) 679.

[55] N. Kitanine, J. M. Maillet, and V. Terras, Nucl. Phys. B 567 (2000) 544.

[56] N. Kitanine, J. M. Maillet, N. A. Slavnov, and V. Terras, Nucl. Phys. B 641 (2002) 487.

[57] N. Kitanine, J. M. Maillet, N. A. Slavnov, and V. Terras, Nucl. Phys. B 642 (2002) 433.

[58] N. Kitanine, J. M. Maillet, N. A. Slavnov, and V. Terras, J. Phys. A 35 (2002) L385 .

[59] N. Kitanine, J. M. Maillet, N. A. Slavnov, and V. Terras, J. Phys. A 35 (2002) L753.

[60] N. Kitanine, J. M. Maillet, N. A. Slavnov, and V. Terras, Nucl. Phys. B 712 (2005) 600.

[61] N. Kitanine, J. M. Maillet, N. A. Slavnov, and V. Terras, Nucl. Phys. B 729 (2005) 558.

[62] N. Kitanine, J. M. Maillet, N. A. Slavnov, and V. Terras, J. Phys. A 38 (2005) 7441.

[63] N. Kitanine, J. M. Maillet, N. A. Slavnov, and V. Terras, J. Stat. Mech. L09002 (2005).

[64] N. Kitanine, J. M. Maillet, N. A. Slavnov, and V. Terras, On the algebraic Bethe Ansatz approach to the correlation functions of the $X X Z$ spin-1/2 Heisenberg chain, In Recent Progress in Solvable lattice Models, RIMS Sciences Project Research 2004 on Method of Algebraic Analysis in Integrable Systems, RIMS, Kyoto, Kokyuroku, 1480 (2006) 14; hep-th/0505006.

[65] N. Kitanine, K. Kozlowski, J. M. Maillet, N. A. Slavnov, V. Terras, J. Stat. Mech. P01022 (2007).

[66] N. Kitanine, K. K. Kozlowski, J. M. Maillet, G. Niccoli, N. A. Slavnov, V. Terras , J. Stat. Mech. (2007) P10009.

[67] K. K. Kozlowski, J. Stat. Mech. (2008) P02006. 
[68] N. Kitanine, K. Kozlowski, J. M. Maillet, G. Niccoli, N. A. Slavnov, V. Terras, J. Stat. Mech. (2008) P07010.

[69] R. J. Baxter, Exactly Solved Models in Statistical Mechanics, Academic Press, New York (1982).

[70] F. C. Alcaraz, M. N. Barber, M. T. Batchelor, R. J. Baxter and G. R. W. Quispel, J. Phys. A 20 (1987) 6397.

[71] N. Yu Reshetikhin, Lett. Math. Phys. 7 (1983) 205.

[72] N. Yu Reshetikhin, Sov. Phys. JETP 57 (1983) 691.

[73] M. T. Batchelor, R. J. Baxter, M. J. O’Rourke, C. M. Yung, J. Phys. A 28 (1995) 2759.

[74] S. Niekamp, T. Wirth, H. Frahm, J. Phys. A 42 (2009) 195008.

[75] W. Galleas, Nucl. Phys. B 790 (2008) 524.

[76] N. Kitanine, J. Phys. A: Math. Gen. 34 (2001) 8151.

[77] O. A. Castro-Alvaredo, J. M. Maillet, J. Phys. A 40 (2007) 7451.

[78] V. V. Bazhanov, Yu G. Stroganov, J. Stat. Phys. 59 (1990) 799.

[79] R. J. Baxter, V. V. Bazhanov and J. H. H. Perk, Int. J. Mod. Phys. B4 (1990) 803.

[80] R. J. Baxter, J. Stat. Phys. 57 (1989) 1.

[81] G. Albertini, B. M. McCoy and J. H. H. Perk, Adv. Study in Pure Math. 19 (1989) 1.

[82] G. Albertini, B. M. McCoy and J. H. H. Perk, Phys. Lett. A 135 (1989) 159.

[83] G. Albertini, B. M. McCoy and J. H. H. Perk, Phys. Lett. A 139 (1989) 204.

[84] R. J. Baxter, J. H. H. Perk and H. Au-Yang, Phys. Lett. A 128 (1988) 138; H. Au-Yang and J. H. H. Perk, Onsager's star triangle equation: Master key to the integrability, in Adv. Stud. in Pure Math. 19 (1989) Kinokuniya-Academic.

[85] R. J. Baxter, Phys. Lett. A 133 (1989) 185.

[86] H. Au-Yang, B. M. McCoy, J. H. H. Perk, S. Tang, and M. Yan, Phys. Lett. A 123 (1987) 219.

[87] B. M. McCoy, J. H. H. Perk, S. Tang, and C. H. Sah, Phys. Lett. A 125 (1987) 9.

[88] H. Au-Yang, B. M. McCoy, J. H. H. Perk, and S. Tang, Algebraic Analysis 1 M. Kashiwara and T. Kawai eds Academic Press, New York (1988).

[89] V. O. Tarasov Phys. Lett. A 147 (1990) 487.

[90] R. J. Baxter, J. Stat. Phys. 117 (2004) 1.

[91] G. Niccoli and J. Teschner, J. Stat. Mech. (2010) P09014.

[92] G. Niccoli, Nucl. Phys. B 835 (2010) 263.

[93] G. Niccoli, JHEP (2011) 1103:123.

[94] G. von Gehlen, N. Iorgov, S. Pakuliak and V. Shadura, J. Phys. A: Math. Gen. 39 (2006) 7257.

[95] G. von Gehlen, N. Iorgov, S. Pakuliak, V. Shadura and Yu Tykhyy, J. Phys. A: Math. Theor. 40 (2007) 14117.

[96] G. von Gehlen, N. Iorgov, S. Pakuliak, V. Shadura and Yu Tykhyy, J. Phys. A: Math. Theor. 41 (2008) 095003.

[97] G von Gehlen, N Iorgov, S Pakuliak, V Shadura, J. Phys. A: Math. Theor. 42 (2009) 304026.

[98] N. Grosjean and G. Niccoli, The $\tau_{2}$-model and the chiral Potts model revisited: Completeness of Bethe equations originated from Sklyanin SOV, J. Stat. Mech. (2012) P10025.

[99] M. Gaudin , Phys. Rev. A 4 (1971) 386.

[100] E. K. Sklyanin, J. Phys. A: Math. Gen. 21 (1988) 2375.

[101] I. V. Cherednik, Theor. Math. Phys. 61 (1984) 977.

[102] P. P. Kulish and E. K. Sklyanin, J. Phys. A: Math. Gen. 24 (1991) L435.

[103] L. Mezincescu and R. Nepomechie, Int. J. Mod. Phys. A 6 (1991) 5231.

[104] P. P. Kulish and E. K. Sklyanin, J. Phys. A: Math. Gen. 25 (1992) 5963.

[105] S. Ghoshal and A. B. Zamolodchikov, Int. J. Mod. Phys. A 9 (1994) 3841.

[106] S. Ghoshal and A. B. Zamolodchikov, Int. J. Mod. Phys. A 9 (1994) 4353.

[107] F. Smirnov, J. Phys. A: Math. Gen. 31 (1998) 8953.

[108] O. Babelon, J. Phys. A 37 (2004) 303.

[109] M. Jimbo, T. Miwa, F. Smirnov, Lett. Math. Phys. 96 (2011) 325.

[110] M. Jimbo, T. Miwa, F. Smirnov, J. Phys. A 42 (2009) 304018.

[111] H. Boos, M. Jimbo, T. Miwa, F. Smirnov, Hidden Grassmann Structure in the XXZ Model IV: CFT limit, arXiv:0911.3731

[112] M. Jimbo, T. Miwa, F. Smirnov, Nucl. Phys. B 852 (2011) 390.

[113] M. Jimbo, T. Miwa, F. Smirnov, Fermionic screening operators in the sine-Gordon model, arXiv:1103.1534. 
[114] A. B. Zamolodchikov, Pis. Zh. Eksp. Teor. Fiz. 25 (1977) 499; A. B. Zamolodchikov, Comm. Math. Phys. 55 (1977) 183.

[115] A. B. Zamolodchikov, Al. B. Zamolodchikov, Ann. Phys. 120 (1979) 253.

[116] M. Karowski and H. J. Thun, Nucl. Phys. B 130 (1977) 295.

[117] V. E. Korepin, Comm. Math. Phys. 76 (1980) 165.

[118] G. Mussardo, Phys. Rep. 218 (1992) 215.

[119] O. Babelon, D. Bernard, F. Smirnov, Comm. Math. Phys. 182 (1996) 319.

[120] O. Babelon, D. Bernard, F. Smirnov, Comm. Math. Phys. 186 (1997) 601.

[121] A. B. Zamolodchikov, Int. J. Mod. Phys. A 3 (1988) 743.

[122] A. B. Zamolodchikov, Adv. Stud. Pure Math. 19 (1989) 641.

[123] Al. B. Zamolodchikov, Nucl. Phys. B 348 (1991) 619.

[124] R. Guida, N. Magnoli, Nucl. Phys. B 471 (1996) 361.

[125] M. A. Virasoro, Phys. Rev. D 1 (1970) 2933.

[126] A. A. Belavin, A. M. Polyakov, A. B. Zamolodchikov, Nucl. Phys. B 241 (1984) 333.

[127] P. Ginsparg, Applied Conformal Field Theory, in: Fields, Strings and Critical Phenomena, Les Houches Lecture Notes 1988, eds. E. Brézin and J. Zinn-Justin, Elsevier, New York (1989).

[128] J. L. Cardy, Conformal Invariance and Statistical Mechanics, in Fields, Strings and Critical Phenomena, ed. E. Brézin and J. Zinn-Justin, Les Houches (1988), Session XLIX North-Holland, Amsterdam (1990) 169.

[129] P. Di Francesco, P. Mathieu and D. Sénéchal, Conformal Field Theory, Springer, New York (1997).

[130] M. Karowski, P. Weisz, Nucl. Phys. B 139 (1978) 455.

[131] F. A. Smirnov, Physica A 3 (1988) 743.

[132] F. A. Smirnov, Form Factors in Completely Integrable Models of Quantum Field Theory, World Scientific, (1992).

[133] F. A. Smirnov, Nucl. Phys. B 337 (1990) 156.

[134] A. Fring, G. Mussardo and P. Simonetti, Nucl. Phys. B 393 (1993) 413.

[135] A. Fring, G. Mussardo and P. Simonetti, Phys. Lett. B 307 (1993) 83.

[136] A. Koubek and G. Mussardo, Phys. Lett. B 311 (1993) 193.

[137] G. Mussardo and P. Simonetti, Int. J. Mod. Phys. A 9 (1994) 3307.

[138] A. Koubek, Nucl. Phys. B 428 (1994) 655.

[139] G. Delfino and G. Mussardo, Nucl. Phys. B 455 (1995) 724.

[140] G. Delfino, P. Simonetti and J. L. Cardy, Phys. Lett. B 387 (1996) 327.

[141] H. Babujian, A. Fring, M. Karowski and A. Zapletal, Nucl. Phys. B 538 (1999) 535.

[142] S. Lukyanov and A. Zamolodchikov, Nucl. Phys. B 607 (2001) 437.

[143] H. Babujian and M. Karowski, Nucl. Phys. B 620 (2002) 407.

[144] H. Babujian and M. Karowski, J. Phys. A: Math. Gen. 35 (2002) 9081.

[145] G. Delfino, J. Phys. A: Math. Gen. 37 (2004) R45.

[146] J. L. Cardy and G. Mussardo, Nucl. Phys. B 340 (1990) 387.

[147] A. Koubek, Nucl. Phys. B 435 (1995) 703.

[148] F. Smirnov, Nucl. Phys. B 453 (1995) 807.

[149] M. Jimbo, T. Miwa, Y. Takeyama, Counting minimal form factors of the restricted sine-Gordon model, arXiv:math-ph/0303059v6.

[150] G. Delfino and G. Niccoli, Nucl. Phys. B 707 (2005) 381.

[151] G. Delfino and G. Niccoli, J. Stat. Mech. (2005) P04004.

[152] G. Niccoli, Descendant Operators in Massive Integrable Quantum Field Theories, Ph.D. Thesis, SISSA (2005).

[153] G. Delfino and G. Niccoli, JHEP 05 (2006) 035.

[154] G. Delfino and G. Niccoli, Nucl. Phys. B 799 (2008) 364.

[155] G. Delfino, Nucl. Phys. B 807 (2009) 455.

[156] A. G. Izergin and V. E. Korepin, Dokl. Akad. Nauk SSSR 259 (1981) 76.

[157] A. G. Izergin and V. E. Korepin, A lattice model related to the nonlinear Schroedinger equation, arXiv:0910.0295.

[158] I.S. Gradshteyn and I.M. Ryzhik; Alan Jeffrey, Daniel Zwillinger, editors. Table of Integrals, Series, and Products, seventh edition. Academic Press (2007).

[159] S. Pakuliak, V. Rubtsov and A. Silantyev, J. Phys. A: Math. Theor. 41 (2008) 295204. 
[160] D. Levy-Bencheton, G. Niccoli and V. Terras, Antiperiodic dynamical 6-vertex model II: Form factors by separation of variables, to appear.

[161] D. Levy-Bencheton, V. Terras, private communications.

[162] J.-S. Caux, J. M. Maillet, Phys. Rev. Lett. 95 (2005) 077201.

[163] J.-S. Caux, R. Hagemans, J. M. Maillet, J. Stat. Mech. (2005) P09003.

[164] R. G. Pereira, J. Sirker, J.-S. Caux, R. Hagemans, J. M. Maillet, S. R. White, I. Affleck, Phys. Rev. Lett. 96 (2006) 257202.

[165] R. Hagemans, J.-S. Caux, J. M. Maillet, Proceedings of the "Tenth Training Course in the Physics of Correlated Electron Systems and High-Tc Superconductors”, Salerno, Oct 2005, AIP Conference Proceedings 846 (2006) 245.

[166] R. G. Pereira, J. Sirker, J.-S. Caux, R. Hagemans, J. M. Maillet, S. R. White, I. Affleck, J. Stat. Mech. (2007) P08022.

[167] J. Sirker, R. G. Pereira, J.-S. Caux, R. Hagemans, J. M. Maillet, S. R. White, I. Affleck, Physica B 403 (2008) 1520.

[168] J. S. Caux, P. Calabrese and N. A. Slavnov, J. Stat. Mech. (2007) P01008.

[169] F. Bloch, Phys. Rev. 50 (1936) 259.

[170] J. S. Schwinger, Phys. Rev. 51 (1937) 544.

[171] O. Halpern and M. H. Johnson, Phys. Rev. 55 (1938) 898.

[172] L. Van Hove, Phys. Rev. 95 (1954) 249.

[173] L. Van Hove, Phys. Rev. 95 (1954) 1374.

[174] W. Marshall and S. W. Lovesey, Theory of Thermal Neutron Scattering, Oxford: Academic (1971).

[175] R. Balescu, Equilibrium and Nonequilibrium Statistical Mechanics, New York: Wiley (1975). 